October 1989

EVALUATION OF THE CONTRIBUTION OF FALL

CHINOOK SALMON REARED AT COLUMBIA RIVER HATCHERIES TO THE PACIFIC SALMON FISHERIES

Final Report

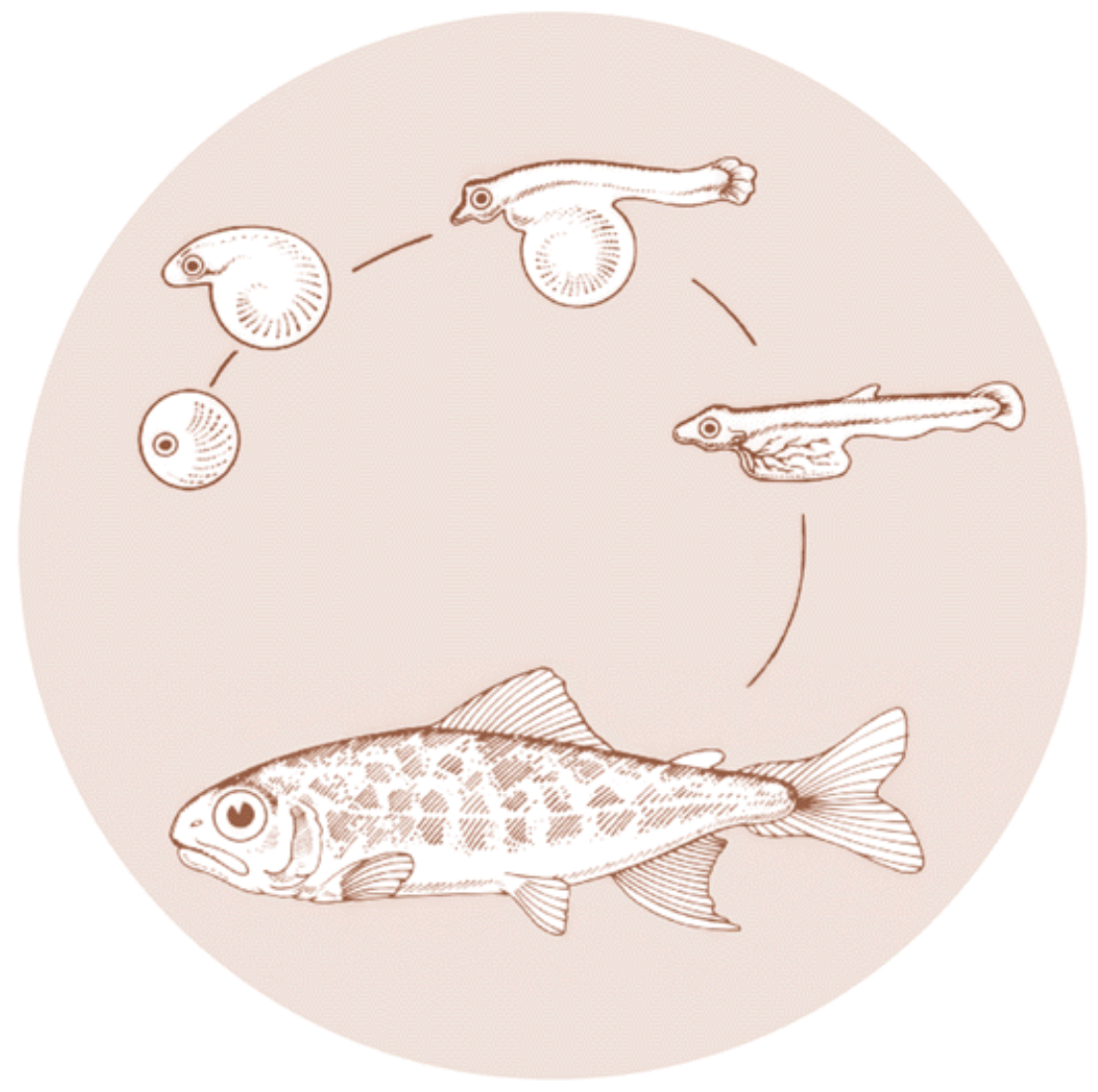

DOE/BP-39638-4 
This report was funded by the Bonneville Power Administration (BPA), U.S. Department of Energy, as part of BPA's program to protect, mitigate, and enhance fish and wildlife affected by the development and operation of hydroelectric facilities on the Columbia River and its tributaries. The views of this report are the author's and do not necessarily represent the views of BPA.

This document should be cited as follows:

Vreeland, Robert R., National Marine Fisheries Service, Evaluation of the Contribution of Fall Chinook Salmon

Reared at Columbia River Hatcheries to the Pacific Salmon Fisheries, Final Report to Bonneville Power

Administration, Portland, OR, Contract 84-AI-39638, Project 79-2, 126 electronic pages (BPA Report DOE/BP-39638-4)

This report and other BPA Fish and Wildlife Publications are available on the Internet at:

\section{http://www.efw.bpa.gov/cgi-bin/efw/FW/publications.cgi}

For other information on electronic documents or other printed media, contact or write to:

Bonneville Power Administration

Environment, Fish and Wildlife Division

P.O. Box 3621

905 N.E. 11th Avenue

Portland, OR 97208-3621

Please include title, author, and DOE/BP number in the request. 


\title{
EVALUATION OF THE CONTRIBUTION OF FALL CHINOOK SALMON REARED AT COLUMBIA RIVER HATCHERIES TO THE PACIFIC SALMON FISHERIES
}

\author{
Final Report
}

Prepared by:

Robert R. Vreeland

National Marine Fisheries Service

Environmental and Technical services Division

Prepared for:

U.S. Department of Energy

Bonneville Power Administration

Environment, Fish and Wildlife

PO Box 3621

Portland, Oregon 97208

Project No. 79-2

Contract No. DE-AI79-84BP39638

October 1989 
LIST OF FIGURES . . . . . . . . . . . . . . . . . . . . . . . . . i i

LIST OF TABLE . . . . . . . . . . . . . . . . . . . . . . iii

LIST OF APPENDIX TABLES . . . . . . . . . . . . . . . . . V V

ABSTRACT .......................... . . . . . . . 1

INTRODUCTION . . . . . . . . . . . . . . . . . . . 2

METHODS AND MATERIALS . . . . . . . . . . . . . . . . . 4

Assumption ....................... . 4

Sampling Fish for Tagging . . . . . . . . . . . . 5

Tagging Procedures . . . . . . . . . . . . . . 10

Prerelease Sampling .. . . . . . . . . . . . 11

Releases ....................... 12

Downstream Migration Sampling . . . . . . . . . . . 12

Fishery Sampling ..................... 13

Rearing Facility and Adjacent Stream Sampling . . . . . . 13

Fishery Contribution Estimation . . . . . . . . . . 15

Benefit/Cost Estimation . . . . . . . . . . . . 15

RESULTS . . . . . . . . . . . . . . . . . . . . . . . 16

Releases .. . . . . . . . . . . . . . 16

Downstream Migrant Recoveries . . . . . . . . . . . . 16

Fishery and Age Distribution of Catch . . . . . . . . . 16

Fishery Contribution . . . . . . . . . . . . . . . . 48

Rearing Facility and Adjacent Stream Returns . . . . . . 70

Total Survival . . . . . . . . . . . . . . . 74

Benefit/Cost .................... 74 
DISCUSSION . . . . . . . . . . . . . . . . . 88

Downstream Migrant Recoveries .............. 88

Fishery and Age Distribution of Catch . . . . . . . . . 89

Fishery Contribution Estimation . . . . . . . . . . . 92

Rearing facility and Adjacent Stream Returns........ 95

Total Survival . . . . . . . . . . . . . . 98

Benefit/Cost Estimation . . . . . . . . . . . . . 99

SUMMARY AND CONCLUSIONS . . . . . . . . . . . . . . . 100

ACKNOWLEDGMENTS . . . . . . . . . . . . . . . . . . 105

LITERATURE CITED . . . . . . . . . . . . . . . . 106

APPENDIX TABLES 1 - 43 . . . . . . . . . . . . . . . . . . . . . 114

APPENDIX A Rearing and release data by brood and facility . . . 238

APPENDIX B Values of fishery contribution by brood and facility . 524 
Figure 1.--Columbia River facilities participating in the fall chinook study................ . . 6

Figure 2. -- Front view of the NMFS fish sampler . . . . . . . . 7

Figure 3.--Side view of the WDF incremental fish sampler . . . . 7

Figure 4.--Fish upwelling into the rear of the WDF sampler.. $\quad \mathbf{8}$

Figure 5.--Water jets forcing fish down a discharge arm of the WDF sampler... . . . . . . . . . . . . . 9

Figure 6.-Ports and zones sampled for tagged fall chinook salmon of Columbia River origin........ . 14

Figure 7.-Proportion of fall chinook salmon form Columbia River rearing facilities caught in Pacific coast fisheries by area of catch and brood year.... .

Figure $8 .--$ Proportion of fall chinook salmon from Columbia River rearing facilities caught in Pacific coast fisheries by age of catch and brood year, 19781981

Figure $9 .--$ Proportion of fall chinook salmon from Columbia River rearing facilities caught in Pacific coast fisheries by age of catch for all brood years and facilities combined and by operating agency...

Figure 10.--Fishery contribution per 1000 releases by brood year

Figure 1l.--Fishery contribution of the 1978-brood fall chinook salmon from Columbia River rearing facilities by facility . . . . . . . . . . . . . .

Figure 12.--Fishery contribution of the 1979-brood fall chinook salmon from Columbia river rearing facilities by facility . . . . . . . . . . . . . . . .

Figure $13 .--F i s h e r y$ contribution of the 1980-brood fall chinook salmon from Columbia River rearing facilities by facility . . . . . . . . . . . . . .

Figure 14 --Fishery contribution of the 1981-brood fall chinook salmon from Columbia River rearing facilities by facility . . . . . . . . . . . . . . . . 
Figure 15. --Proportion of the fishery contribution of 1978-brood fall chinook salmon from Columbia River rearing facilities attributable to each facility . .

Figure 16 --Proportion of the fishery contribution of 1979-brood fall chinook salmon from Columbia River rearing facilities attributable to each facility . . .

Figure 17 --Proportion of the fishery contribution of 1980-brood fall chinook salmon from Columbia River rearing facilities attributable to each facility . . . .

Figure 18 --Proportion of the fishery contribution of 1981-brood fall chinook salmon from Columbia River rearing facilities attributable to each facility . .

Figure 19 --Proportion of fall chinook salmon from Columbia River rearing facilities escaping the fisheries by age of return and brood year, 1978 - 1981 . . . . . .

Figure 20 --Proportion of fall chinook salmon from Columbia River rearing facilities escaping the fisheries by age of return for all brood years and facilities combined and by operating agency . . . . . . . . . . 
Table 1. -Releases of 1978-brood fall chinook salmon from Columbia River facilities in 1979 . . . . . . . . .

Table 2.--Releases of 1979-brood fall chinook salmon from Columbia River facilities in 1980 . . . . . . .

Table 3.--Releases of 1980-brood fall chinook salmon from Columbia River facilities in 1981 . . . . . . . .

Table 4.--Releases of 1981-brood fall chinook salmon from Columbia River facilities in 1982 . . . . . . . .

Table 5.--Recoveries of tagged 1978-brood fall chinook from Columbia River rearing facilities during sampling of downstream migrants at various locations on the Columbia River in 1979 and 1980 . . . . . . . .

Table 6 --Recoveries of tagged 1979-brood fall chinook from Columbia River rearing facilities during sampling of downstream migrants at various locations on the Columbia River in 1980 and 1981 . . . . . . . . . .

Table 7 --Recoveries of tagged 1980-brood fall chinook from Columbia River rearing facilities during sampling of downstream migrants at various locations on the Columbia River in 1981 . . . . . . . . . . . . . .

Table 8 -Recoveries of tagged 1981-brood fall chinook from columbia River rearing facilities during sampling of downstream migrants at various locations on the Columbia River in 1982 . . . . . . . . . . .

Table 9 - Estinated recoveries of tagged 1978-brood fall chinook from Columbia River rearing facilities to Pacific coast fisheries by facility . . . . . . . . .

Table 10 - Estimazed recoveries of tagged 1979-brood fall chinook from Columbia River rearing facilities to Pacific coast fisheries by facility . . . . . . . .

Table 11 --Estimated recoveries of tagged 1980 -brood fall chinook from Columbia River rearing facilities to Pacific coast fisheries by facility. . . . . . . . .

Table 12 -Estimated recoveries of tagged 1981-brood fall chinook from Columbia River rearing facilities to Pacific coast fisheries by facility . . . . . . . . 
Table 13.--Tagged catch and contribution of 1978-brood fall chinook salmon to all Pacific coast fisheries by rearing

facility . . . . . . . . . . . . . . .

Table 14. --Tagged catch and contribution of 1979-brood fall chinook salmon to all Pacific coast fisheries by rearing facility . . . . . . . . . . . . . .

Table 15.--Tagged catch and contribution of 1980-brood fall chinook salmon to all Pacific coast fisheries by rearing facility . . . . . . . . . . . . . . .

Table 16. -Tagged catch and contribution of 1981-brood fall chinook salmon to all Pacific coast fisheries by rearing facility . . . . . . . . . . . . . . .

Table 17.--Catch, escapement, and survival data for all broods of tagged fall chinook salmon combined (1978-1981) by rearing facility . . . . . . . . . . .

Table 18. --Catch, escapement, and survival data for tagged 1978-brood fall chinook salmon from Columbia River rearing facilities................. 76

Table $19 .--$-Catch, escapement, and survival data for tagged 1979-brood fall chinook salmon from Columbia River rearing facilities...................

Table 20 .--Catch, escapement, and survival data for tagged 1980-brood fall chinook salmon from Columbia River rearing facilities . . . . . . . . . . . . . . .

Table 21.--Catch, escapement, and survival data for tagged 1981-brood fall chinook salmon from Columbia River rearing facilities . . . . . . . . . . . . . . .

Table 22.--Cost of rearing and benefits for the 1978-brood fall chinook salmon at Columbia River rearing facilities

Table 23.--Cost of rearing and benefits for the 1979-brood fall chinook salmon at Columbia River rearing facilities

Table 24.--Cost of rearing and benefits for the 1980-brood fall chinook salmon at Columbia River rearing facilities

Table 25.--Cost of rearing and benefits for the 1981-brood fall chinook salmon at Columbia River rearing facilities 
Page

Appendix Table 1. --Proportions of tagged 1978-brood fall chinook from Columbia River rearing facilities recovered in Pacific coast fisheries by facility and area of $\mathrm{c} \mathrm{a} \mathrm{t} \mathrm{ch...............}$

Appendix Table 2. --Proportions of tagged 1979-brood fall chinook from Columbia River rearing facilities recovered in Pacific coast fisheries by facility and area of $\mathrm{c} \mathrm{a} \mathrm{t} \mathrm{ch................}$

Appendix Table 3. --Proportions of tagged 1980-brood fall chinook from Columbia River rearing facilities recovered in Pacific coast fisheries by facility and area of c a $\mathrm{t} \mathrm{ch}$................ .

Appendix Table 4. --Proportions of tagged 1981-brood fall chinook from Columbia River rearing facilities recovered in Pacific coast fisheries by facility and area of $\mathrm{c} \mathrm{a} \mathrm{t} \mathrm{ch}$...............

Appendix Table 5. --Proportions of tagged 1978-brood fall chinook from Columbia River rearing facilities recovered in all Pacific coast fisheries combined by age of recovery . . . . . . . . . . . . . . .

Appendix Table 6. --Proportions of tagged 1979-brood fall chinook from Columbia River rearing facilities recovered in all Pacific coast fisheries combined by age of recovery.................. .

Appendix Table 7.--Proportions of tagged 1980-brood fall chinook from Columbia River rearing facilities recovered in all Pacific coast fisheries combined by age of recovery,. . . . . . . . . . . . . .

Appendix Table 8.-- Proportions of tagged 1981-brood fall chinook from Columbia River rearing facilities recovered in all Pacific coast fisheries combined by age of recovery . . . . . . . . . . . . . . . . .

Appendix Table 9. --Estimated recoveries of tagged 1978-brood fall chinook from Columbia River rearing facilities to Pacific coast fisheries by facility, tag code, and catchyear . . . . . . . . . . . . . . . 
Appendix Table 10.--Estimated recoveries of tagged 1979-brood fall chinook from Columbia River rearing facilities to Pacific coast fisheries by facility, tag code, and catchyear . . . . . . . . . . . . . .

Appendix Table 11.-.Estimated recoveries of tagged 1980-brood fall chinook from Columbia River rearing facilities to Pacific coast fisheries by facility, tag code, and catchyear . . . . . . . . . . . . . . .

Appendix Table 12...Estimated recoveries of tagged 1981-brood fall chinook from Columbia River rearing facilities to Pacific coast fisheries by facility, tag code, and catchyear . . . . . . . . . . . . . . . . .

Appendix Table 13.-Contribution of 1978-brood fall chinook salmon to all Pacific coast fisheries by hatchery and tag code, . . . . . . . . . . . . . . . .

Appendix Table 14.-.Contribution of 1979-brood fall chinook salmon to all Pacific coast fisheries by hatchery and tag code....................

Appendix Table 15.-Contribution of 1980-brood fall chinook salmon to all Pacific coast fisheries by hatchery and tag code. . . . . . . . . . . . . . . . . .

Appendix Table 16..-Contribution of 1981-brood fall chinook salmon to all Pacific coast fisheries by hatchery and tag code. . . . . . . . . . . . . . . . . . .

Appendix Table 17.--Tagged returns of 1978-brood fall chinook salmon in 1980 to Columbia River rearing facilities and adjacent streams. . . . . . . . . . . .

Appendix Table 18.--Tagged returns of 1978-brood fall chinook salmon in 1981 to Columbia River rearing facilities and adjacent streams . . . . . . . . . . . . .

Appendix Table 19.--Tagged returns of 1978 -brood fall chinook salmon in 1982 to Columbia River rearing facilities and adjacent streams . . . . . . . . . . . . .

Appendix Table 20.--Tagged returns of 1978-brood fall chinook salmon in 1983 to Columbia river rearing facilities and adjacent streams. . . . . . . . . . . .

Appendix Table 21.--Tagged returns of 1979-brood fall chinook salmon in 1981 to Columbia River rearing facilities and adjacent streams 
Appendix Table 22.--Tagged returns of 1979-brood fall chinook salmon in 1982 to Columbia River rearing facilities and adjacent streams . . . . . . . . . . . . . .

Appendix Table 23.--Tagged returns of 1979-brood fall chinook salmon in 1983 to Columbia River rearing facilities and adjacent streams . . . . . . . . . . . . . . .

Appendix Table 24.--Tagged returns of 1979-brood fall chinook salmon in 1984 to Columbia River rearing facilities and adjacent streams . . . . . . . . . . . . . .

Appendix Table 25.--Tagged returns of 1979-brood fall chinook salmon in 1985 to Columbia River rearing facilities and adjacent streams. . . . . . . . . . . . . .

Appendix Table 26.--Tagged returns of 1980-brood fall chinook salmon in 1982 to Columbia River rearing facilities and adjacent streams . . . . . . . . . . . . .

Appendix Table 27.--Tagged returns of 1980-brood fall chinook salmon in 1983 to Columbia River rearing facilities and adjacent streams . . . . . . . . . . . . . .

Appendix Table 28.--Tagged returns of 1980-brood fall chinook salmon in 1984 to Columbia River rearing facilities and adjacent streams . . . . . . . . . . . .

Appendix Table 29.--Tagged returns of 1980-brood fall chinook salmon in 1985 to Columbia River rearing facilities and adjacent streams . . . . . . . . . . . . . . .

Appendix Table 30.--Tagged returns of 1980 -brood fall chinook salmon in 1986 to Columbia River rearing facilities and adjacent streams......... . . . . . .

Appendix Table 31.--Tagged returns of 1981-brood fall chinook salmon in 1982 to Columbia River rearing facilities and adjacent streams. . . . . . . . . . . .

Appendix Table 32.--Tagged returns of 1981-brood fall chinook salmon in 1983 to Columbia River rearing facilities and adjacent streams... . . . . . . . . . .

Appendix Table 33.- Tagged returns of 1981-brood fall chinook salmon in 1984 to Columbia River rearing facilities and adjacent streams . . . . . . . . . . . . . . 
Appendix Table 34.--Tagged returns of 1981-brood fall chinook salmon in 1985 to Columbia River rearing facilities and adjacent streams . . . . . . . . . . . .

Appendix Table 35.--Tagged returns of 1981-brood fall chinook salmon in 1986 to Columbia River rearing facilities and adjacent streams

Appendix Table 36.--Tagged returns of 1978-brood fall chinook salmon to Columbia River rearing facilities and adjacent

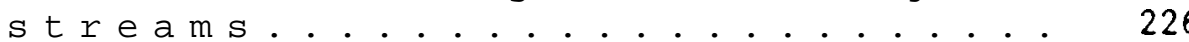

Appendix Table 37.--Tagged returns of 1979-brood fall chinook salmon to Columbia River rearing facilities and adjacent

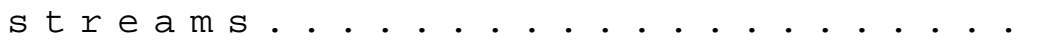

Appendix Table 38.--Tagged returns of 1980 -brood fall chinook salmon to Columbia River rearing facilities and adjacent

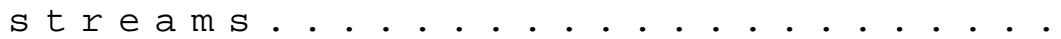

Appendix Table 39.--Tagged returns of 1981-brood fall chinook salmon to Columbia River rearing facilities and adjacent

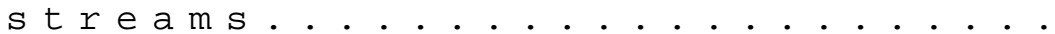

Appendix Table 40.--Proportions of tagged 1978-brood fall chinook from Columbia River rearing facilities returning to the facilities and adjacent streams by age of recovery . . . . . . . . . . . . . . . . .

Appendix Table 41.--Proportions of tagged 1979-brood fall chinook from Columbia River rearing facilities returning to the facilities and adjacent streams by age of recovery . . . . . . . . . . . . . . . . .

Appendix Table 42.--Proportions of tagged 1980-brood fall chinook from Columbia River rearing facilities returning to the facilities and adjacent streams by age of recovery . . . . . . . . . . . . . . . . .

Appendix Table 43.--Proportions of tagged 1981-brood fall chinook from Columbia River rearing facilities returning to the facilities and adjacent streams by age of recovery 


\section{ABSTRACT}

In 1979 this study was initiated to determine the distribution, contribution, and value of artificially propagated fall chinook salmon from the Columbia River. Coded wire tagging of hatchery fall chinook salmon began in 1979 with the 1978 brood and was completed in 1982 with the 1981 brood of fish at rearing facilities on the Columbia River system. From $\mathbf{1 8}$ to 20 rearing facilities were involved in the study each brood year. Nearly 14 million tagged fish, about 48 of the production, were released as part of this study over the four years, 1979 through 1982. Sampling for recoveries of these tagged fish occurred from 1980 through 1986 in the sport and commercial marine fisheries from Alaska through California, Columbia River fisheries, and returns to hatcheries and adjacent streams.

The fish from this study were recovered primarily in the British Columbia and Washington ocean fisheries and the Columbia River commercial gillnet fisheries. The average proportions recovered by fishery are 3.68 for Alaska, 42.48 for British Columbia, 27.0 for Washington, 4.78 for Oregon, 0.38 for California, 21.98 for Columbia River, and 0.18 for foreign fisheries. The contribution rates by fishery varied among the broods. The contribution was greater to the Alaska and British Columbia fisheries in the 1980 and 1981 broods.

It is estimated that $\mathbf{1}, 020,800$ fall chinook salmon from rearing facilities participating in this study were recovered in the Pacific coast fisheries from 1980 through 1986. The average fishery recovery per 1000 fish released is 2.9. The recovery rates by brood are 3.3, 4.7, 1.9, and 2.0 per 1000 releases for the 1978 through 1981 broods respectively. There is considerable variation among rearing facilities within a brood. The lowest fishery recovery per 1000 releases is 0.1 for Elokomin Hatchery, $1978 \mathrm{brood}$. The greatest ratio is $12.7 \mathrm{fish}$ per 1000 releases from Spring Creek Hatchery, 1979 brood.

Rearing facility and adjacent stream returns in general followed the same trend as the fishery contributions. If a release from a particular rearing facility had a grater than average fishery contribution, the hatchery return was also grater than the average.

Total survival (fishery recovery and spawning escapement) for all broods combined is 0.338 . Survivals by brood are $0.33,0.46,0.28$, and 0.25 z for the 1978 through 1981 broods respectively. Survivals among rearing facilities ranged from 0.018 (Elokomin Hatchery, 1978 brood) to nearly 1.58. (Spring Creek Hatchery, 1979 brood).

The total cost of rearing the four broods of fall chinook salmon is $\$ 6,334,000$. The value of the fishery recovery from the rearing facilities participating in this study is estimated at $\$ 36,242,000$, for a benefit to cost ratio of $5.7 / 1$. The benefit to cost ratios by brood are $7.8 / 1,9.8 / 1,3.6 / 1$, and $2.5 / 1$ for the 1978 through 1981 brood respectively. The range among rearing facilities is from $0.2 / 1$ (Litile White Hatchery, 1978 brood) to 26.6/1 (Spring Creek Hatchery, 1979 brood) . 


\section{INTRODUCTION}

Historically the Columbia River system produced the worlds' largest runs of chinook salmon (Oncorhynchus tshawytscha) (Van Hyning 1973). Craig and Hacker (1950) estimated that when white men first arrived on the Columbia River, 50,000 Indians were catching 18 million pounds of salmon. Salmon fishing was one of the inducements for settling the Columbia Basin. It provided lucrative employment for thousands and directly or indirectly provided the means for many to make their fortunes (Hume 1893). In 1832, Captain Nathaniel J. Wyeth came to Oregon from Massachusetts, established Fort Hall on the Lewis River (a tributary of the lower Columbia River), and became the first person to commercially fish for salmon on the Columbia River (Cobb 1930). The first canning operation on the Columbia River was started by William

Hume and his partners in 1867 (Craig and Hacker 1950). Between 1875 and the mid-1920's, the annual catch of chinook salmon in the Columbia River averaged 20 to 40 million pounds (Van Hyning 1973). The greatest canned salmon pack of 40 million pounds occurred in 1883 (Hume 1893; Van Hyning 1973). The first notable report of sport fishing on the Columbia was in 1889 when Rudyard Kipling reported his fishing experiences on the willamette River near willamette Falls (Cobb 1930). In the 1920's it was not uncommon to see 500 boats, containing 1 to 6 sport salmon fisherman, on a day in April or May on the willametre River (Cobb 1930).

Bean et al. (1938) estimated the annual retail value of commercially caught fish from the Columbia River was about $\$ 10$ million. Richards (1968) estimated the gross value of commercially harvested chinook salmon by non-Indian commercial fisheries in the Columbia River in 1965 was $\$ \mathbf{4 . 4}$ million. The value of the Indian catch of chinook was

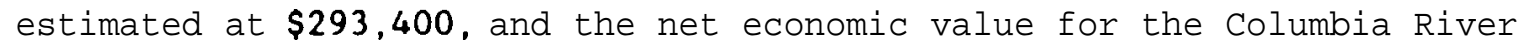
sport catch of chinook salmon was $\$ 2.3$ million (Richards 1968). Helsing (1972) estimated that in 1968 the total value of the anadromous salmonid resources from the Columbia River was $\$ 34.8 \mathrm{million}$, and the net benefit to the national economy produced by Columbia River anadromous fish was $\$ 15.0$ million.

Initial declines in catches of chinook salmon on the Columbia River first occurred in the 1880's. Catches fluctuated from 1890 through the mid-1920's and then began a steady decline. These reduced catches were caused by over fishing and degradation and destruction of spawning grounds due to logging, mining. and irrigation practices (Craig and Hacker 1950; Van Hyning 1973). Construction of dams on the main stem of the Columbia River for irrigation and electrical power in the 1930's added to the decline of populations of chinook salmon (Craig and Hacker 1950). Populations of chinook salmon where further impacted when dam construction proliferated from the 1950's though the 1970's (Schoeneman et al. 1961; Raymond 1979).

As mitigation for loss of natural spawning habitat, a massive artificial rearing program for salmon and steelhead (Oncorhynchus sp.) was undertaken on the Columbia River. Salmon and steelhead hatcheries were constructed or renovated under the Lower Columbia River Development Program (Laythe 1948). Under the Columbia River Fisheries Development Program alone $\$ 31.5$ million dollars were expended for hatchery 
construction and another $\$ \mathbf{1 3 7 . 5}$ million for operation and maintenance and hatchery evaluation programs between 1949 through 1987 (Delarm and Smith 1988). The Bureau of Reclamation funded construction of hatcheries on tributaries to the Mid-Columbia River as mitigation for Grand Coulee Dam (Fish and Hanavan 1948). The U.S. Army Corps of Engineers funded construction and renovation of hatcheries as mitigation for Snake River and Columbia River dams (Hansen, Ewing, and Martin 1980; Tuss 1982: Crateau 1989). Private and public utilities constructed spawning and rearing facilities in Washington and Idaho as mitigation for dams throughout the upper Columbia River Basin (Meekin and Moser 1966; Allen 1970; Reingold 1978).

The previously mentioned salmon and steelhead culture programs were not the first to occur on the Columbia River system. The first salmon hatchery was constructed on the Clackamas River (an Oregon tributary to the Columbia River) in 1876. The first salmon hatchery in the state of Washington was built in 1895 on the Kalama River (Cobb 1930).

Studies to determine the impact of the artificial salmon culture programs began in the 1920's. Rich and Holmes (1928) reported on marking experiments with chinook salmon on the Columbia River from 1916 to 1927. These studies were the first to indicate the extended migration of Columbia River chinook salmon as far north as Southeastern Alaska. Snyder (1923) reported on recoveries of chinook salmon marking experiments at Mount Shasta Hatchery on the Sacramento River and Fall Creek Hatchery on the Klamath River in California. The Pacific Marine Fisheries Commission sponsored the first coastwide salmon marking and recovery experiment at hatcheries in California, Oregon, and Washington in 1950 and 1951 (Hallock, Warner, and Fry 1952; Junge and Bayliff 1955; Van Hyning 1973). A major hatchery evaluation program began in 1961 on the Columbia River. Sockeye salmon (O. nerka) at Leavenworth National Fish Hatchery on a tributary of the Wenatchee River were fin and maxillary marked for four years (Wahle, Koski, and Smith 1979). Marking of four brood years of fall chinook salmon began at Columbia River hatcheries in 1962 (Wahle and Vreeland 1978). In 1966 three brood years of coho salmon (O.kisutch)were marked at Columbia River hatcheries (Wahle, Vreeland, and Lander 1974). In 1965 a three year study of hatchery contribution to the fisheries was begun at hatcheries rearing coho salmon on tributaries to Puget Sound in Washington (Senn 1970a; 1970b; 1971). With the advent of the coded wire tag (CwT) (Bergman et al. 1968), a multitude of studies have been conducted to determine the fishery contribution and survival of salmon from hatcheries on the Pacific coast of North America (Pacific Marine Fisheries Commission 1988).

In 1979 the Bonneville Power Administration (BPA) began funding a study to determine the distribution, contribution, and value of fall chinook salmon raised at columbia Rirer rearing facilities. This tagging study provides data to determine the effectiveness of hatcheries constructed as mitigation for hydroelectric developments. In addition, these data can aid fishery agencies in planning further measures to protect, mitigate, and enhance salmon runs on the Columbia River. This information is important to regulating bodies, such as the Pacific Fishery Management Council, charged with negotiating, setting, and adjusting fishing seasons, location, and limits. The last comprehensive 
examination of fishery contributions of fall chinook from Columbia River hatcheries was completed over fifteen years ago (Wahle and Vreeland 1978). After that fin-marking study was completed, it became part of the basis for current fishery regulations. Since completion of that study, new rearing facilities have been built, existir: facilities renovated, changes in sport and commercial fisheries have occurred, and hatchery practices have been altered.

\section{METHODS AND MATERIALS}

The National Marine Fisheries Service (NMFS) coordinated this study among three fishery agencies: U.S. Fish and Wildlife Service (USFWS), Oregon Department of Fish and Wildlife (ODFW), and Washington Department of Fisheries (WDF). The objectives of this study were to determine the distribution, fishery contribution, survival, and value of the production of fall chinook salmon from each rearing facility on the Columbia River system to Pacific coast salmon fisheries. To achieve these objectives fish from each hatchery were given a distinctive CWT.

Assumptions

Marking all fall chinook salmon at a rearing facility would have been cost prohibitive, thus a sample of fish was selected for tagging. To apply the recovery of tagged fish to the total population of fish from a facility requires seven assumptions. These assumptions are:

1. Fish to be tagged are representatively sampled and receive the same treatment as untagged $f$ ish before and after marking.

2. Tagged fish are identifiable throughout their life.

3. Tagged and untagged fish have the same growth and survival rates and maturity schedules.

4. Tagged and untagged fish have the same marine distribution and vulnerability to the catch.

5. The probability of a fish being sampled is independent of whether it is tagged or untagged.

6. All tagged fish in the sample are recognized and correctly identified and reported.

7. The tag is not duplicated for any other study.

The appropriateness of the estimating procedures used in this study and the conclusions which can be drawn are dependent on the validity of these assumptions. In some cases precautions were taken and data collected to check the assumptions or adjust for deviations from them (Assumptions 1, 2, 3,6, and 7). In other cases the assumptions had to 
be accepted on faith in order to proceed with the study (Assumptions 4 and 5).

Sampling Fish for Tagging

From 18 to 20 facilities rearing fall chinook salmon on the Columbia River were included in this study each year (Figure 1). Sampling devices developed by WDF and MMFS were used to obtain a random sample of fish for tagging. A sample of fish was removed from all rearing environments at a facility. The rigorous sampling procedure was followed to ensure the validity of Assumption 1 (fish to be tagged are representatively sampled). The intent of the sampling devices was to place as little handling stress on the fish as possible and accomplish the sampling in a timely manner. A fish pump removed fish from the raceways at a facility and delivered them to the samplers. The MMFS sampler was an "A" shaped inclined plane table (Figure 2). Fish and water from the pump upwelled at the top of the "A" (A, Figure 2), flowed over a perforated plate to remove excess water, and off the foot of the table to a return trough. The foot of the table was divided into 20 sections of equal width (B, Figure 2). In theory, the fish spread evenly across the table and 5 of the population passing over the sampler traveled through each of the 20 sections. A chute the width of one of the sections, attached to the foot of the table, allowed fish to pass over the return trough and into a receptacle. These fish were retained for tagging. The chute could be the width of one, two, or more sections to remove a 58, 108, or larger sample.

In the incremental sampler (Figure 3) (Foster 1981), developed by WDF, fish and water from the fish pump upwelled at the rear of the sampler (Figure 4). Excess water was removed as the fish slid across a bar grate to a "Y" shaped discharge pipe (Figure 5). Water jets, activated by a solenoid, forced fish down one of the two discharge arms of the $Y$. To obtain a 58 sample, one jet sprayed for 5 of the time and the other for 958 of the time. The jets frequently activated alternately throughout the sampling process (for example every 20 seconds), creating as random a sample as possible. The 5 sample was retained for tagging.

Both samplers were tested to determine the percentage of fish removed during the sampling process. The tests were not completely successful because it appeared the samplers operated best with a large sample size and a steady supply of fish. Inconsistent results were obtained when small test lots of fish were sampled. Neither device was adequately tested to determine if each fish had an equal chance of being sampled or if the sample fish were representative of the entire population. It was assumed that the sampling process produced the closest possible approximation to a random sample.

Sampling of fall chinook salmon populations at each facility was accomplished with the sampling devices where possible. At some facilities this was not possible due to the limited amount of sampling equipment, inability to reach the rearing environments with the fish pumps, or rearing environments that would not allow crowding and sampling the entire population of fish (large release ponds). In the 


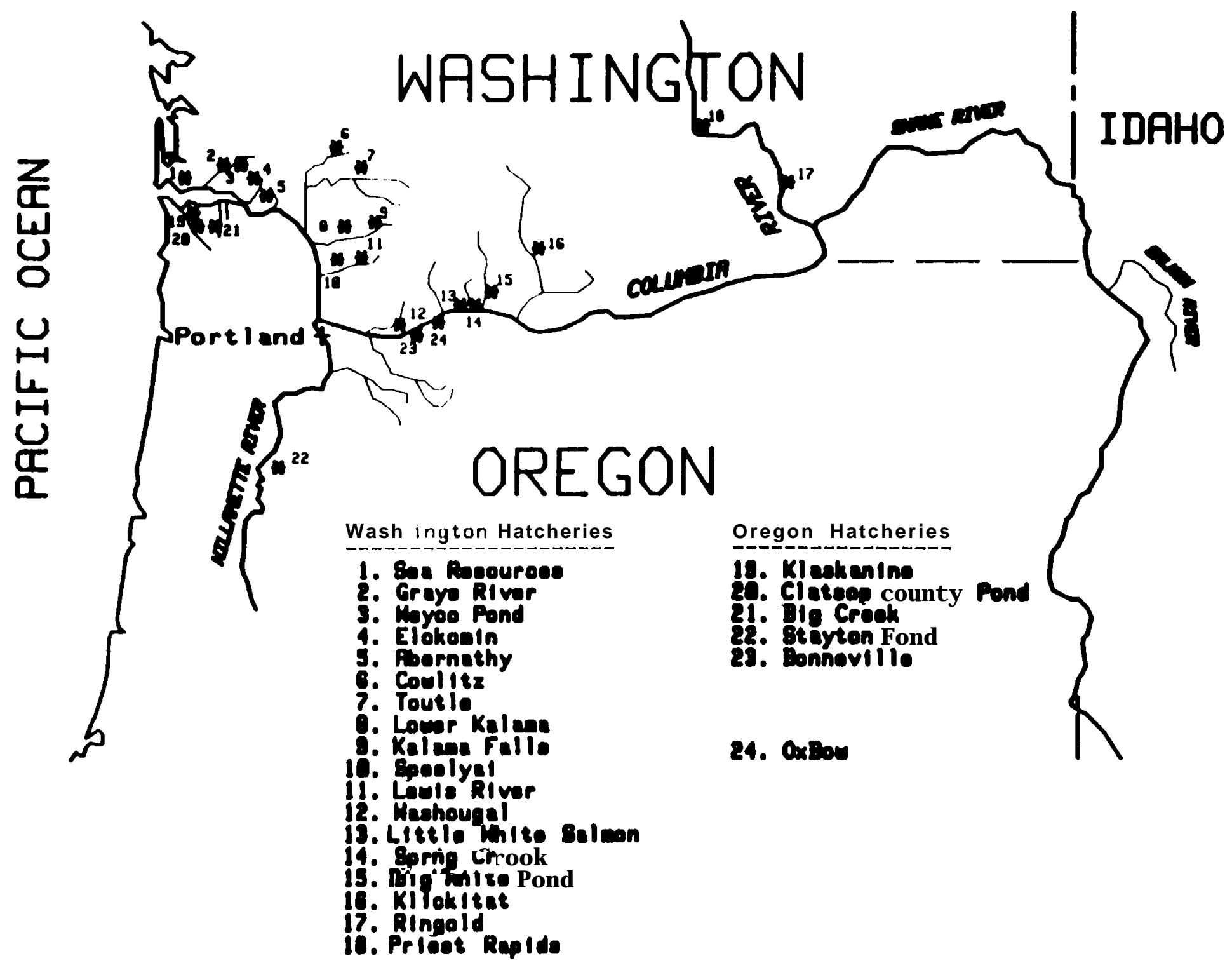

figure 1. - COLUMBIA RIVER FACILITIES PARTICIPATING IN THE FALL CHINOOK STUDY 


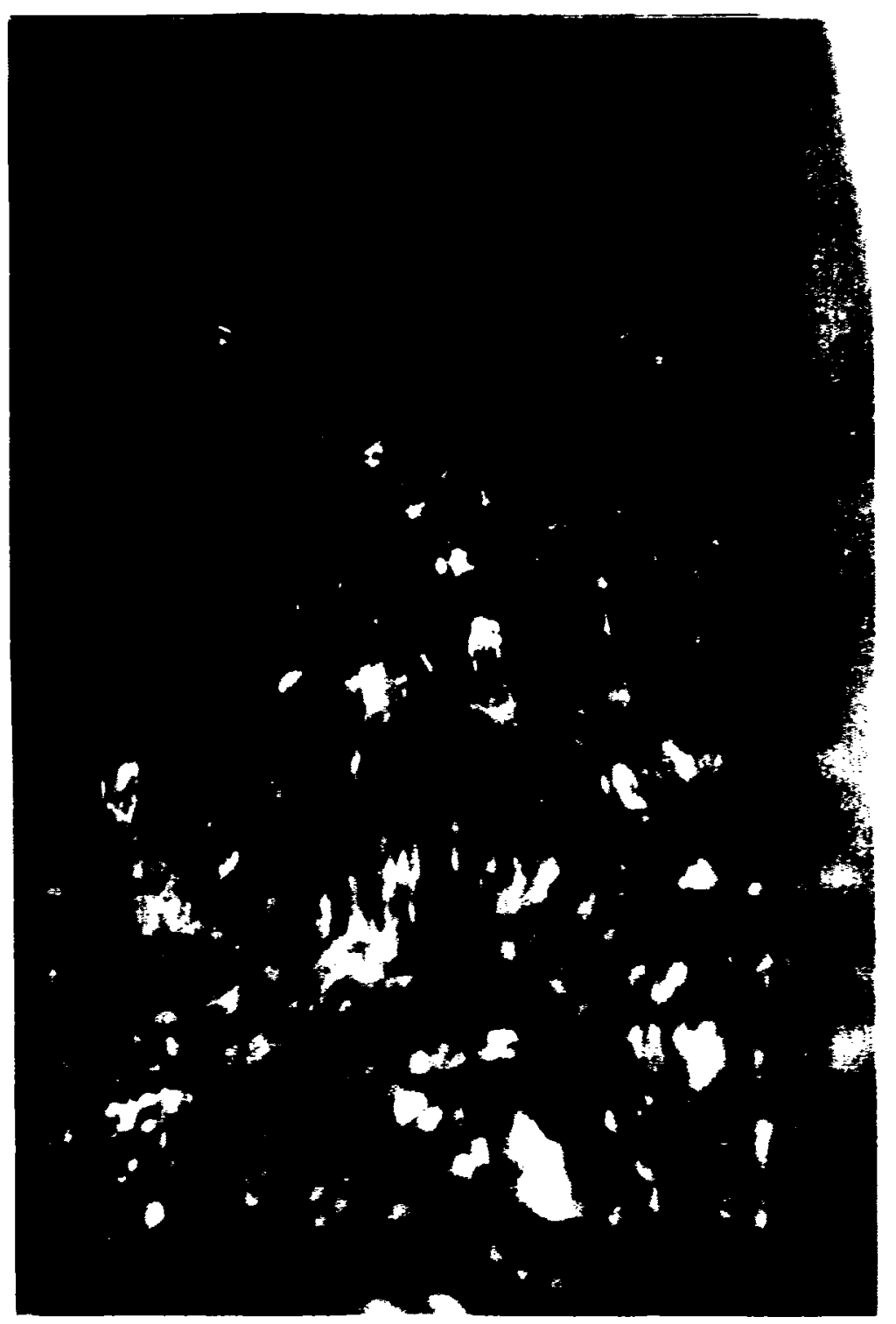

Figure 4.--Fish upwelling into the rear of the WDF sompler. 


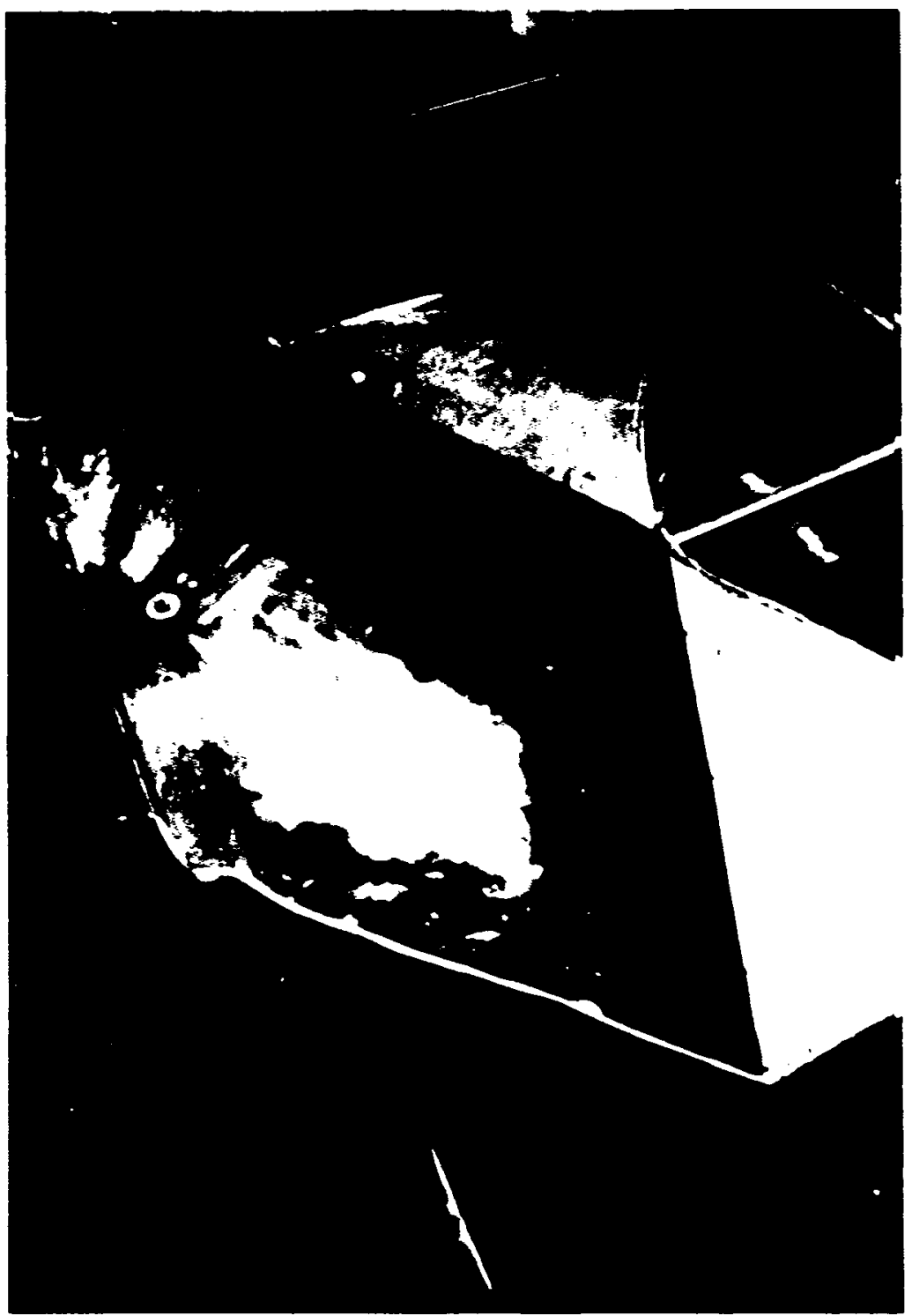

Figure 5.--Hater jets forcing fish down o discharge arm of the WDF sompler. 
large release ponds (Stayton Pond for example), the fish to be tagged were seined from the periphery of the pond. Where the entire population of a rearing environment could be crowded into a small area but the mechanical sampling devices could not be used, fish to be tagged were obtained by handling the entire population with a dip net and setting aside a systematic sample (one in every 20 nets of fish for example). The sampling method used at each facility is presented in ie hatchery data forms in Appendix A.

In 1979, the first year of the study, 58 of the production of fall chinook salmon at each rearing facility was removed for tagging. In some cases, the 5s sample was not large enough to meet the goal of examining the contribution to the fisheries of the production from a facility. In these situations, additional fish were removed and a separate tag code applied to these fish. In subsequent years (1980 1982), the proportion removed for tagging at facilities with large fall chinook salmon productions was reduced to 2.5 (Spring Creek, Little White Salmon, Bonneville, Big Creek, and Klaskanine hatcheries) to reduce the cost of tagging and the time required.

At some facilities, fish were sampled as much as two months prior to tagging. This was done at the time of transferring fish to release ponds at several WDF facilities. At the time of transfer, the fish were. too small to be tagged with a standard sized CUT. The fish to be tagged were held separately in raceways and mixed with the appropriate populations of untagged flsh after tagging. In most cases, sampling occurred just prior to tagging. The times of sampling and tagging are presented in the hatchery data forms in Appendix A.

Tagging Procedures

Personnel from the participating agencies tagged a portion of the fall chinook salmon production at most Columbia River facilities rearing this species. Tagging did not occur at some facilities because of lack of funds (Clatsop County Ponds, 1978 brood), logistical constraints (Big White Pond, 1979 brood), and emergency releases of fish (Lower Kalama Hatchery, 1978 brood; Clatsop County Ponds, 1979 brood; and OxBow, 1980 brood). Tagging of fall chinook salmon for purposes other than the hatchery evaluation study occurred at some facilities (Spring Creek, Bonneville, and Big Creek hatcheries). Fall chinook salmon at each of the facilities participating in this study received a distinctive mark consisting of an adipose fin clip and insertion of a uricue CWT in the snout. This insured Assumption 7 (no duplication of mark) was valid since the reuse of a code is not allowed (Pacific Marine Fisheries Commission 1988). From 2.5 to 5 percent of the production at each facility was randomly selected for tagging.

ODFW and USFWS personnel used a mobile tagging unit constructed by MMFS with WA funds to tag fish a their facilities. WDF used their own tagging equipment. Tagging began in mid February each year and extended into early July. At ODFW and USFWS facilities, fish were anesthetized, the adipose fin removed with bent-nose scissors, and a co\&d wire tag was inserted into the cartilage in the snout of the fish. 
At WDF facilities, the fish wre ascthotsed and the dipoes $f$ in rowoved, than the fish were later ancthotized agaln and the ters inserted.

Tagging of the 1978-brood fall chinook salmon bogan in, Fobruary of 1979. The flanl year of tagging for this stuxy. wn 1982, when the 1981-brood fish wre tagged. Over the four brood yearo. 1978 through 1981, marly 15 million fall chinook salmon were taged. The nubars tagged for each brood yeat were $4,379,300,3,009,900,3,660,500$, and $, 3,651,300$

reopectively. The m-bara of $\mathrm{fish}$ tagged at each facllity are presented in the hatchery data fores in Appendix A.

Except for the 1978-brood fall chinook at ODFi and usris facllities, tagged fish were returned to the population of untaged fish from which they ans. This helped to insure Ascuption 1 (equal treatment of tagged and untagged fish after tagsins) wa valid.- Soparate bolding of tagged fish after tagging occurred in 1979 because It was thought that untagged fish would have a competitive advantage over tagged fish while the tagged fish were recovering from marking stress. This could result in untagged fish being luger than tagged fish at release, which atht influence survival. However, separate holding of tagged and untagged fish could not be accomplished at all facilities due to limited rearing.. space. In addition several problem erose with the soparate holding. In som cases, tagged fish were held in substantially different rearing cuvironents (concrete raceways vs. dirt bottom release ponds). . The differentenvironmes could have caused differences in erowth and: survival. At Spring Creak Hatchery, there was son Widanee thet own when the holding envirounents were the san for the resged and uneasged fish, size differences occurred. The tagged-fish for the earlitent release at Spring Creek appeared to be smaller than the untagged flah. (337 fish $/ \mathrm{kg}$ for tagged fish and $276 \mathrm{fish} / \mathrm{kg}$ for untagged fish for the March 1979 rel ease). This could have been due to the closeness of tagging to release and the tagged fish not recoverifs sufficiently from the stress of tagging to make up the growth advantage-gainad by-the untagged fish. In a later release, the tagged fish appeared to be larger then the untagged fish (172 fish/kg for tagged fish and 185 $\mathrm{fish} / \mathrm{kg}$ for the untagged fish for the April 1979 ;release). This could have been we to Ilghter flsh densities in the raged fish racewaye than the untagged fish raceways. The observed differences could also hwe been due to sampling error. To prevent any bias caused by separate bolding, tagged fish were mixed with the untagged populations from which they cen for the 1979 through 1981 broods.

Prerelease Sampling

During the tagging operation at each facility, tagging supervisors randomly removed a minimum of 2,000 tagged fish. These fish were held separately and were examined at the time of the facility's production release to determine tag retention at release. Tagging supervisors examined mortalities in the ponds after tagging to determine the mber of tagged fish dying prior to release. In some cases, the mortalities of tagged fish and the tag loss estimate were subtracted from the nuber 
of fish tagged to estimate the releases of tagged fish. In these cases, the total release was estimated from facility records.

Just prior to release, fall chinook populations at most participating facilities were sampled to determine the tagged to untagged ratios. This was accomplished at ODFU and USFUS facilities by passing the populations of one fourth to one half of the raceways through the NMFS sampler. A 10 sample was removed and passed though the sampler again to obtain a 18 sample of the population of the raceways. The fish in the 1. sample were examined for tags, and a tagged to untagged ratio was developed. The sample ratio was expanded to estimate the tagged and untagged fish released. At WDF facilities, hatchery records, the proportion of fish removed for tagging, and sampling for the tagged to untagged ratio at release were all used to estimate the tagged, untagged, and total releases. The specific method for estimating release numbers for each hatchery each year is contained in the hatchery data forms in Appendix $A$.

Releases

Production releases of fall chinook salmon generally occurred in May and. June at the facilities. Spring Creek Hatchery made releases in March, April, May, and August of each year. September releases occurred at Speelyai, Nashougal, and Cowlitz hatcheries for the 1978, 1980, and 1981 broods. A group of 1978-brood fall chinook salmon was released in October from Cowlitz Hatchery. Cowlitz Hatchery also aade releases of yearling fall chinook salmon, from the 1979 brood, in March and April of 1981.

Releases generally occurred directly from the rearing facility with some notable exceptions. No releases were made directly from Stayton Pond. All fish were transported to release sites throughout the Willanette River basin. The 1979-brood fall chinook salmon at Cowlitz Hatchery were transported to tributaries outside the Cowlitz drainage due to the eruption of Mt St. Helens which caused high silt loads in the lower Cowlitz River. The 1979-brood fall chinook salmon from OxBow Hatchery were transported by truck upriver to McNary Dam, placed in a barge, transported downstream in the barge, and released below Bonneville Dam. A group of 1980-brood fish from Spring Creek Hatchery was transported to a tributary of the Columbia River above John Day Dam for release. Two groups of 1981-brood fish from Spring Creek and Bonneville hatcheries were transported upstream and released into the Umatilla River. The upriver transport programs were conducted to exanine the return location of the transported fall chinook salmon and to determine if Indian fisheries above Bonneville Dam could be enhanced by upriver releases. Detailed release dates and locations are presented in the hatchery data forms in Appendix $A$.

Downstream Migration Sampling

Research personnel from MAFS, Northwest Fisheries Center sampled the 
Columbia River estuary and marine waters near the mouth of the Columbia River during the downstream migration of the four broods of fall chinook salmon (Dawley et al. 1986). The purposes of this sampling were to: 1) define migrational characteristics of marked salmonid stocks from release site through the estuary, 2) provide data to assist in evaluating different hatchery production techniques within a release year, and 3) determine juvenile survival to the estuary for selected stocks and compare this survival with fishery contribution and hatchery returns of these stocks.

In general sampling crews collected fish in beach and purse seines one to seven days per week from March through September. Juvenile salnonids captured were sorted from the catch and examined for marks and brands. Fish with an adipose fin clip were sacrificed to obtain CWT's. In addition data were maintained of fish sizes, movement rates, and feeding rates of tagged groups recovered at Jones Beach (Dawley et al. 1986).

In 1979 and 1980, juvenile salnonids passing six dams on the Columbia and Snake Rivers were collected and examined. This was done as part of the smolt migration timing and transportation research being conducted by MFS (Raymond and Sims 1980; Sims et al. 1981). At McNary and John Day Dams, fish passing through the fingerling collection facilities were examined for marks and brands. NiFs personnel sacrificed up to 75 adipose marked fish per day at each facility and extracted CWr's. In 1979 sampling at McNary and John Day dams took place from April 9 to August 24 and April 4 to December 3, respectively. In 1980 sampling took place at the same two dams from April 3 to August 15 and April 14 to December 3, respectively. This sampling effort is of interest to this study because tagged fall chinook salmon fron Priest Rapids Hatchery migrated past these two dams.

\section{Fishery Sampling}

State fishery agencies and the Department of Fisheries and Oceans in British Columbia provided personnel to examine sport and commercial catches of salmon at major marine fishing ports from Alaska through California (Figure 6). The goal of this sampling was to examine 208 of the catch of salmon for marks. When a salmon with a missing adipose fin was observed, the samplers recorded lengths, in some case took scales for aging, and removed the snout of the fish. The fish snouts were sent to head dissection labs where the wire tags were extracted and read, and the information recorded. Commercial and sport catches of salmon on the Columbia River were sampled in the same manner. Marine and Columbia River fishery sampling occurred throughout this study. Recoveries of the 1978-brood began in 1980. Fishery recoveries of the 1981 brood were completed in 1986 .

Rearing Facility and Adjacent Stream Sampling

Personnel from WDF, ODFW, and USFWS in most cases examined all returning fall chinook at participating facilities for the absence of fins. 


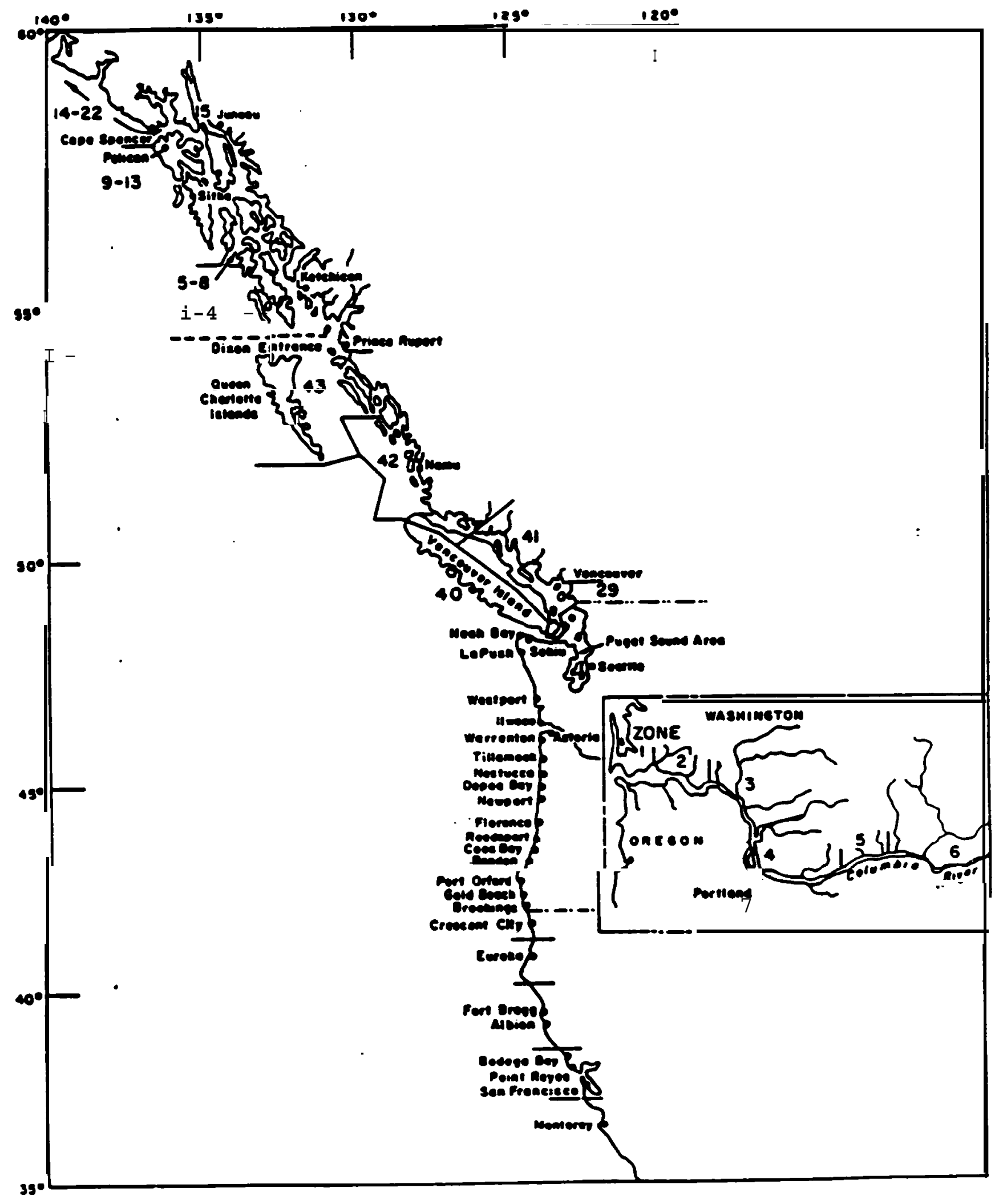

Figure 6.--Ports and zones sampled for togged foll chinook salmon of Columbia River origin.

14 
Biological data were collected from untagged returns at a predetermined systematic sampling rate. Samplers removed the snout of all fish with a missing adipose fin. The biological data were collected to estimate the ages of untagged returning fish. The age structure for tagged and untagged fish can be compared to determine if tagging changes the age distribution of returns.

Spawning ground surveys on the streams where the rearing facilities are located and on adjacent streams also occurred. Snouts were removed from all salmon suspected of being tagged. Biological data were collected from all tagged fish recovered.

Fishery Contribution Estimation

The contribution of the fall chinook salmon from Columbia River rearing facilities was estimated from the number of tagged fish from these facilities recovered in the Pacific coast fisheries. The sampling agencies expand the number of tags observed in the fishery sampling to the entire catch during the year. The estimated tag recoveries were divided by the proportion of tagged fish at release to estimate the fishery contribution of the fall chinook salmon from each hatchery. The, proportion of tagged fish at release was adjusted for the number of tagged fish removed in the estuary sampling effort. However, no adjustment was made for other potential selective mortality of tagged fish after release. This mortality has been shown to be substantial in past studies where fish were marked by removing fins and maxillary bones (Wahle, Vreeland, and Lander 1974; Wahle and Vreeland 1978). However, the excision of an adipose fin and insertion of a CWT is believed to have negligible impact on survival of salmon after release (Bergman 1968; Eames and Hino 1983; Zajac 1985).

Benefit/Cost Estimation

The benefits of the catch of fall chinook salmon from this study were developed using a method described in an unpublished report by Richards (1987). Values per fish caught were presented in the report for both commercial and sport fisheries for the years 1980 through 1984. The comerclal values were based on average price per pound paid to the fishermen for the fish by area (Alaska, Washington, Oregon, and California). Washington data were used for British Columbia. The sport values were based on the value per fishing trip. These values were weighted by the proportion of fish from each facility caught in each of the fisheries to yield a value of the fish caught from each facility. These values were then multiplied by the total contribution of fish from each facility by brood to yield the benefit of the contribution to the fisheries.

Costs for rearing the 1978 through 1981 broods of fall chinook salmon were provided by the operating agencies. The costs include: fish food, chemicals and drugs, labor, transportation of fish and goods, supplies, equipment, power, and overhead. No capital costs were included. 


\section{RESULIS}

Releases

Nearly 14 million tagged fall chinook salmon from four brood years were released from the participating facilities. Releases were 4,035,100, $2,864,700,3,466,400$, and 3,475,500 for the 1978 through 1981 brood years, respectively. The proportions of tagged fish released each year were $4.4,3.5,3.9$, and 4.1 for the four broods, respectively. Total annual releases ranged from 81 to 92 million fish from the participating facilities (Tables 1-4).

Downstream Migrant Recoveries

Recoveries of most tagged groups occurred in the downstream sampling (Tables 5-8). Recovery numbers ranged from none for some codes to over 2200 for one tag code. Recovery numbers were greatest for the 1978 brood of fish and steadily declined for the other three broods. The recovery numbers are a function of the number of fish tagged, the sampling effort, fish size, migration distance, river flow at the time of recovery, and the survival of the fish to the sampling site. More 1978-brood fall chinook salmon were tagged than for other broods, and the sampling effort was greater in 1979 than in subsequent years. In 1981 and 1982, sampling was limited to Jones Beach, $75 \mathrm{kn}$ upstream from the Columbia River mouth. Estuarine recoveries of most tag groups began within one week of release. The length of time between release and first recovery was a function of the distance between the release and recovery sites and the rate of migration of the fish. The final recoveries of most groups occurred within one to two months after release. In a few instances, recoveries were made a year after the release of the group (Priest Rapids Hatchery, 1978 brood; and Kalama Falls and Lewis River hatcheries, 1979 brood). Recoveries of yearling migrant fall chinook salmon from Priest Rapids Hatchery (17 observed fish) occurred at McNary Dam. The two yearling migrants from Kalama Falls and Lewis River hatcheries were recovered at Jones Beach.

Fishery and Age Distribution of Catch

Fall chinook salmon tagged for this study were caught in fisheries from Alaska to California. Distributions of the catch by brood year and area of catch are presented in Figure 7. Approximately 90 of the catch of fall chinook salmon tagged for this study occurred in three areas: British Columbia, Washington, and the Columbia River. For the four brood years combined, 3.68 of the fish were caught in Alaska fisheries, 42.48 in British Columbia fisheries, 27.08 in Washington fisheries, 4.78 in Oregon fisheries, 0.3 in California fisheries, 21.98 in Columbia River fisheries, and 0.1 as incidental catch in foreign fisheries in the Pacific Ocean off the North American coast. The proportion of the catch recovered by area varies considerably among the brood years. The 
Table 1.--Releases of 1978-brood fall chloook soleon fron Columbla River faclieles in 1979.

\begin{tabular}{|c|c|c|c|c|c|c|c|c|}
\hline Rearine Facllicy & Tas Code & $\begin{array}{l}\text { Taceed Fish } \\
\text { Released }\end{array}$ & $\begin{array}{l}\text { Ad Only Fish } \\
\text { Released }\end{array}$ & $\begin{array}{c}\text { X Tas } \\
\text { Detentica }\end{array}$ & $\begin{array}{l}\text { Dumarted Fisb } \\
\text { Deleased }\end{array}$ & $\begin{array}{c}\text { Tocal } \\
\text { Delease }\end{array}$ & Flah/1b & Dalease Dates \\
\hline ADERUATHY Hatchoxy & $\begin{array}{l}05-04-50 \\
05-04-51\end{array}$ & $\begin{array}{l}63.400 \\
48.900\end{array}$ & $\begin{array}{l}15,000 \\
11,600\end{array}$ & $\begin{array}{l}60.2 \\
\omega 0.2\end{array}$ & $\begin{array}{l}830,600 \\
640,700\end{array}$ & $\begin{array}{l}909,000 \\
701,200\end{array}$ & $\begin{array}{l}93 \\
61\end{array}$ & $\begin{array}{l}04 / 17-05 / 18 \\
04 / 17-05 / 18\end{array}$ \\
\hline DIG CREEX HATCHERY & $07-18-44$ & 224,900 & 26,400 & 99.5 & $4,996,000$ & $3,267,300$ & $\mathbf{e 1}$ & $05 / 21$ \\
\hline DIG WAITE POND & $05-04-43$ & 141.400 & 3,200 & 97.8 & 2.884 .100 & $3,020,700$ & 69 & $05 / 21$ \\
\hline DOMEVILLE HATCHERY & $\begin{array}{l}07-18-42 \\
07-18-43\end{array}$ & $\begin{array}{r}287,900 \\
15,100\end{array}$ & $\begin{array}{r}5.500 \\
200\end{array}$ & $\begin{array}{l}90.1 \\
90.7\end{array}$ & $\begin{array}{r}12,262,400 \\
824,000\end{array}$ & $\begin{array}{r}12,555,000 \\
039,300\end{array}$ & $\begin{array}{l}75 \\
60\end{array}$ & $\begin{array}{r}0 s / 01-0 s / 29 \\
0 s / 21\end{array}$ \\
\hline CONLIT2 HATCHERY & $\begin{array}{l}63-19-42 \\
63-19-51\end{array}$ & $\begin{array}{r}143,600 \\
11,100\end{array}$ & $\begin{array}{c}2,500 \\
-0-\end{array}$ & $\begin{array}{r}98.3 \\
100.0\end{array}$ & $\begin{array}{c}4,678,800 \\
-0-\end{array}$ & $\begin{array}{r}4,624,900 \\
11,100\end{array}$ & $\begin{array}{l}e 5 \\
e 5\end{array}$ & $\begin{array}{l}06 / 27-10 / 16 \\
06 / 27-10 / 16\end{array}$ \\
\hline ELOROMIN HATCHERY & $\begin{array}{l}63-18-56 \\
63-19-56\end{array}$ & $\begin{array}{r}21,100 \\
117,800\end{array}$ & $\begin{array}{c}-0- \\
5.800\end{array}$ & $\begin{array}{r}100.0 \\
95.3\end{array}$ & $\begin{array}{c}-0- \\
2,730,700\end{array}$ & $\begin{array}{r}21,100 \\
2,254,300\end{array}$ & $\begin{array}{l}99 \\
99\end{array}$ & $\begin{array}{l}06 / 15 \\
06 / 15\end{array}$ \\
\hline CRAYS RIVER HATCAERY & $\begin{array}{l}63-16-46 \\
63-18-33 \\
63-19-37\end{array}$ & $\begin{array}{r}73,900 \\
7.600 \\
68,100\end{array}$ & $\begin{array}{c}2,600 \\
-0- \\
-0-\end{array}$ & $\begin{array}{r}90.3 \\
100.0 \\
100.0\end{array}$ & $\begin{array}{c}1,220,000 \\
-0- \\
-0-\end{array}$ & $\begin{array}{r}1,297,300 \\
7,600 \\
68,100\end{array}$ & $\begin{array}{l}92 \\
92 \\
92\end{array}$ & $\begin{array}{l}06 / 09-06 / 12 \\
06 / 09-06 / 12 \\
06 / 09-06 / 12\end{array}$ \\
\hline ralaM FALLS MATCaERY & $63-19-57$ & 214,500 & 3,300 & 90.5 & $3,940,300$ & $4,158,100$ & 177 & $06 / 22-07 / 13$ \\
\hline TLASTMNIME HATCaERY & $07-18-45$ & 246.100 & 28.600 & 89.5 & $5,218,100$ & $3,490,000$ & 11 & $05 / 29$ \\
\hline DLICKITAT HATCHERY & $63-19-69$ & 225.400 & 3.100 & 90.4 & $3,366,400$ & $3,595,500$ & $\boldsymbol{s 0}$ & $05 / 14-06 / 13$ \\
\hline LITTLE MHITE HATCHERY & $\begin{array}{l}05-04-48 \\
05-04-69\end{array}$ & $\begin{array}{l}177,800 \\
264,800\end{array}$ & $\begin{array}{r}8.900 \\
12.700\end{array}$ & $\begin{array}{l}95.2 \\
95.4\end{array}$ & $\begin{array}{l}5,655,500 \\
5,291,100\end{array}$ & $\begin{array}{l}5,842,200 \\
5,560,600\end{array}$ & $\begin{array}{l}111 \\
111\end{array}$ & $\begin{array}{l}06 / 22 \\
06 / 22\end{array}$ \\
\hline
\end{tabular}


Table 1.--(Cont Inuad)

\begin{tabular}{|c|c|c|c|c|c|c|c|c|c|}
\hline Rearins Facllity & Tes code & $\begin{array}{l}\text { Tased Fish } \\
\text { Released }\end{array}$ & $\begin{array}{l}\text { Ad Only Fish } \\
\text { Released }\end{array}$ & $\begin{array}{c}8 \text { Ias } \\
\text { Decention }\end{array}$ & $\begin{array}{l}\text { Unearked Fish } \\
\text { Released }\end{array}$ & $\begin{array}{c}\text { Total } \\
\text { Delesse }\end{array}$ & Fish/1b & Rèlease D & Dates \\
\hline \multirow[t]{4}{*}{ PRIEST RAPIDS BATCHEnY } & $63-18-21$ & 48.100 & 2,000 & $\boldsymbol{x . 0}$ & 776,400 & 026,500 & 76 & & $05 / 23$ \\
\hline & $63-18-57$ & 17,500 & $-0-$ & 100.0 & 267.700 & 285,900 & 77 & & $06 / 28$ \\
\hline & $63-19-58$ & 5,300 & $-0-$ & 100.0 & $-0-$ & 5.300 & 77 & & $06 / 28$ \\
\hline & $63-20-17$ & 82,200 & 700 & 99.2 & $-0-$ & 02,200 & $M$ & & $06 / 28$ \\
\hline SEA RESOURCES HATCAERY & $63-19-18$ & 24,200 & 300 & 98.6 & 957.500 & 902,000 & 112 & $05 / 01-0$ & $05 / 31$ \\
\hline \multirow[t]{2}{*}{ SPEELYAI HATCHERY } & $63-19-20$ & 51,700 & 400 & 99.2 & $-0-$ & 52.100 & 28 & & $09 / 05$ \\
\hline & $63-19-50$ & 104,500 & 3.500 & 96.8 & 78,500 & 186,500 & $\infty$ & & $07 / 19$ \\
\hline \multirow[t]{4}{*}{ SPRIMG CREEK GATCHERY } & $05-04-33$ & 140,900 & 13,600 & 91.2 & $3,568,600$ & $3,723,100$ & s4 & & $05 / 18$ \\
\hline & $05-04-64$ & 135,500 & 19.400 & 07.5 & $4,357,400$ & $4,512,300$ & 87 & & $04 / 20$ \\
\hline & $05-04-45$ & $\$ 5,600$ & 6,300 & 99.9 & $1,141,600$ & $1,203,500$ & 19 & & $08 / 13$ \\
\hline & $05-04-46$ & 246,000 & 13,000 & 95.0 & $9,261,000$ & $10,120,000$ & 125 & & $03 / 20$ \\
\hline STAYTOW POND & $07-18-41$ & 283,800 & 9.400 & 96.8 & $4,398,800$ & $4,692,000$ & 67 & 05107 & $05 / 21$ \\
\hline \multirow[t]{2}{*}{ TOUTLE HATCHaRY } & $63-18-54$ & 12,000 & $-0-$ & 100.0 & $-0-$ & 12,000 & 160 & & $06 / 17$ \\
\hline & $63-19-61$ & 232,100 & 6,000 & 96.0 & 2.619 .500 & $2,757,600$ & 160 & & $06 / 17$ \\
\hline \multirow[t]{2}{*}{ MASBOUGAL GATCaERY } & $63-19-38$ & 97.400 & 8,300 & 96.8 & $4,826,800$ & $4,932,500$ & 78 & $06 / 14$ & $09 / 02$ \\
\hline & $63-19-46$ & 154,500 & $-0-$ & 100.0 & $-0-$ & 154,500 & 78 & $06 / 14$ & $09 / 02$ \\
\hline UEYCO POND & $63-19-39$ & 92.400 & 2,500 & 97.6 & 271.600 & 366,500 & 58 & & $06 / 05$ \\
\hline Total & & $4,035,100$ & 215,400 & & $87,464,900$ & $91,715,400$ & & & \\
\hline
\end{tabular}


Table2.--Releases of 1979-brood fall chlnook salnon fron Colubla River faclities In 1980.

\begin{tabular}{|c|c|c|c|c|c|c|c|c|}
\hline Rearing Faclliey & Ias Code & $\begin{array}{l}\text { Taceed Fish } \\
\text { Released }\end{array}$ & $\begin{array}{l}\text { Ad Oaly Fish } \\
\text { Released }\end{array}$ & $\begin{array}{c}2 \text { Ias } \\
\text { Docencioa }\end{array}$ & $\begin{array}{l}\text { Donarked Flsh } \\
\text { Deleased }\end{array}$ & $\begin{array}{c}\text { Total } \\
\text { Delease }\end{array}$ & Flsh/Lb & Delease Dates \\
\hline \multirow[t]{2}{*}{ ADEUATHY HATCAERY } & $05-06-64$ & 35,200 & 1,100 & א.9 & 466,540 & 502,800 & 59 & $06 / 09-05 / 14$ \\
\hline & $05-06-46$ & 112,500 & 2,400 & 97.9 & $1,360,000$ & $1,474,900$ & 59 & $04 / 09=05 / 14$ \\
\hline BIC CREEK HATCEERY & $07-21-60$ & 143,400 & 2.200 & 90.5 & $6,207,900$ & $6,433,500$ & 78 & $05 / 13$ \\
\hline DOMUEVILLE BATCaERY & $07-21-57$ & 121,100 & 4,400 & 96.5 & $0,967,400$ & $5,072,900$ & 74 & $05 / 20-05 / 20$ \\
\hline \multirow[t]{3}{*}{ COULITZ EATCAERY } & $63-21-56$ & 244,300 & 9.900 & $\mathrm{x} .1$ & $5,671,600$ & $5,926,000$ & 129 & $06 / 03-07 / 11$ \\
\hline & $63-21-59$ & 70,500 & 2,900 & $* .1$ & $1,566,600$ & $1,640,000$ & 219 & $06 / 18-07 / 11$ \\
\hline & $63-21-37$ & 20,700 & 200 & 99.7 & 543,400 & 564,300 & 9 & $03 / 21-06 / 01$ \\
\hline ELOROAIN HATCHERY & $63-20-05$ & 98,400 & 2.100 & 97.9 & $2,310,600$ & $2,411,100$ & $\infty$ & $06 / 19$ \\
\hline GRAYS RIVER BATCHERY & $63-20-43$ & 37.500 & 1,500 & 96.2 & 768,000 & 007,000 & 85 & $06 / 01-06 / 24$ \\
\hline WALAM FALLS BATCaIDXY & $63-21-05$ & 100.400 & 1.500 & 50.5 & $2,299,000$ & $2,400,900$ & 126 & $06 / 13-06 / 24$ \\
\hline DLASTUNINE HATCHERY & $07-21-61$ & 66,300 & 900 & 96.7 & $2,170,500$ & $2,237,700$ & 79 & $06 / 04$ \\
\hline ILICKITAT HATCHERY & $63-19-47$ & 156.100 & 1.600 & 98.0 & $2,901,700$ & $3,139,400$ & 85 & $05 / 27$ \\
\hline LEUIS RIVER HATCHERY & $63-21-60$ & 103.700 & 1.800 & 90.3 & 321,700 & 427,200 & 117 & $02 / 15$ \\
\hline LITTLE MaITE BATCaERY & $05-06-43$ & 162,600 & 1,900 & 9.9 & $\bullet, 611.500$ & $0,776,000$ & 101 & $06 / 10$ \\
\hline LOUER RALAYA BATCBERY & $63-20-06$ & 144.500 & 5,800 & 96.2 & $3,129,500$ & $3,279,800$ & 150 & $06 / 10$ \\
\hline \multirow[t]{2}{*}{ OXBOW HATCHERY } & $07-21-62$ & 49,400 & 900 & 90.3 & $1,115,200$ & $1.165,500$ & 100 & $05 / 27$ \\
\hline & $07-21-63$ & 51,900 & 900 & 90.3 & $1,170,100$ & $1.222,900$ & 100 & $05 / 27$ \\
\hline
\end{tabular}


Table 2.--(Cont Loued)

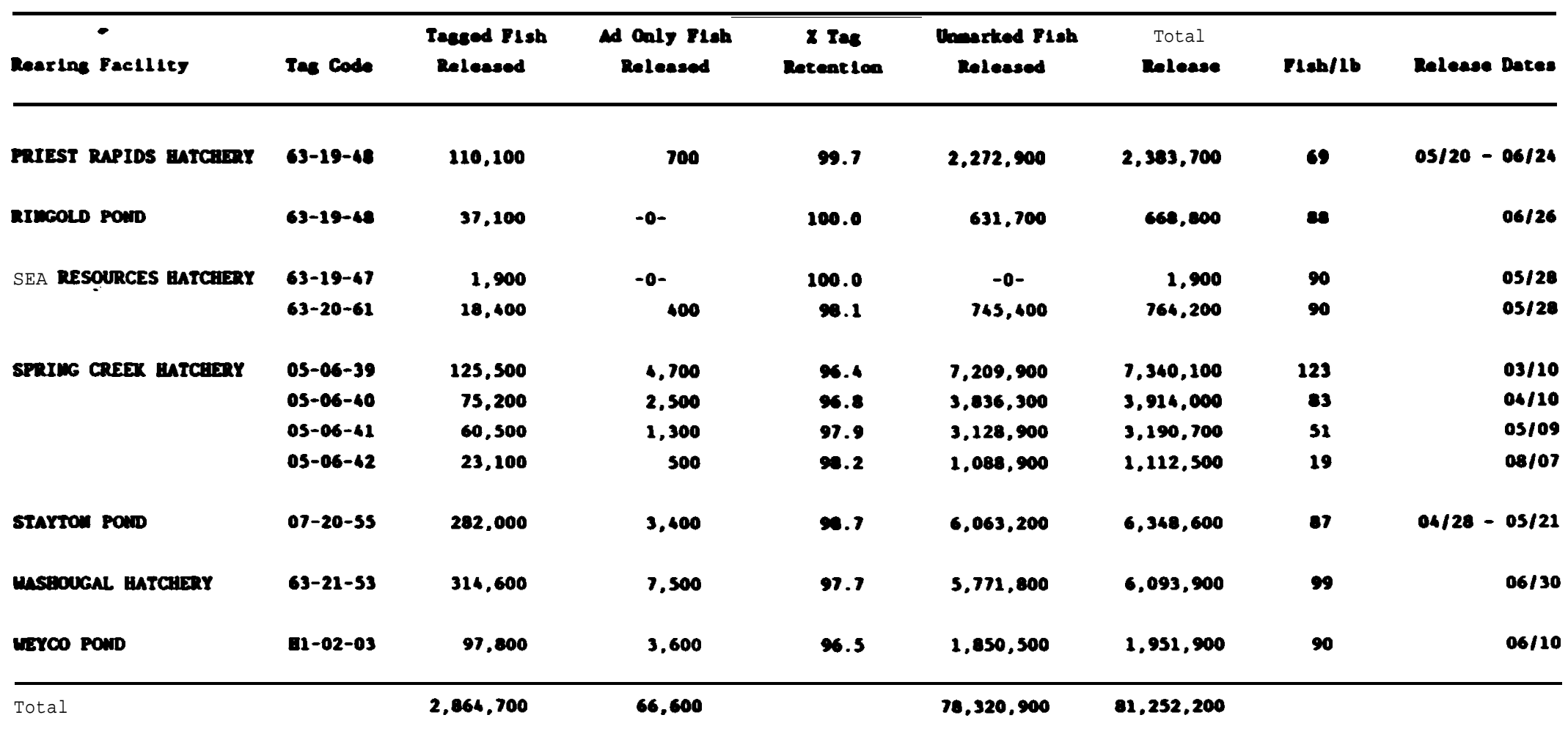


Table 3.--Releases of 1900-brood fall chloook salmon from Coluble River feclities in 1901.

\begin{tabular}{|c|c|c|c|c|c|c|c|c|}
\hline \multirow[b]{2}{*}{ Rearlne Facilley } & \multirow{2}{*}{\multicolumn{2}{|c|}{$\begin{array}{l}\text { Tacesed Flsh } \\
\text { Tae code Released }\end{array}$}} & \multirow{2}{*}{$\begin{array}{l}\text { ad Oaly Flsh } \\
\text { Released }\end{array}$} & \multirow{2}{*}{$\begin{array}{l}\text { Tas } \\
\text { Retearicas }\end{array}$} & \multirow{2}{*}{ 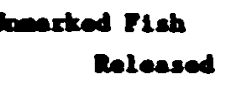 } & \multirow{2}{*}{$\begin{array}{l}\text { Tocal } \\
\text { Delease }\end{array}$} & \multirow[b]{2}{*}{ Flah/L } & \multirow[b]{2}{*}{ Delease Dates } \\
\hline & & & & & & & & \\
\hline \multirow{2}{*}{ ANEPUATEY HATCuST } & $05-07-46$ & 19,100 & 3,300 & es.4 & 278,000 & 300,400 & 69 & $04 / 15$ - 05/26 \\
\hline & $05-07-45$ & 63,500 & 10.600 & 05.7 & 026,700 & 900,000 & 69 & $04 / 15-05 / 26$ \\
\hline \multirow[t]{3}{*}{ DIC CREEX Hatchent } & $07-23-31$ & 50,200 & 1,500 & 97.1 & $1.056,000$ & $1,907,700$ & 77 & $05 / 07=05 / 18$ \\
\hline & $07-23-33$ & $\$ 1.100$ & 1.600 & 92.0 & I. . . - & 1.941 .300 & 77 & $05 / 07-05 / 10$ \\
\hline & $07-23-36$ & 46.000 & 1.400 & 97.0 & $1,69 ., 100$ & $1,745,500$ & 77 & $05 / 07-05 / 10$ \\
\hline \multirow[t]{2}{*}{ BOAUEVILLE HATCHIDYY } & $07-21-56$ & 130,000 & 4,200 & 96.5 & $5,007,400$ & $5,142,200$ & 73 & $04 / 24$ \\
\hline & $07-23-29$ & 75.700 & 2.700 & 96.6 & $3,113,000$ & $3,191,400$ & $\mathbf{0}$ & $05 / 12$ \\
\hline \multirow{2}{*}{ CLATSOP COUATY PODS } & $07-21-50$ & 73,200 & 900 & s.. & $1,726,200$ & 1, 100,500 & 75 & $05 / 25$ \\
\hline & $07-21-59$ & 48,900 & 300 & 9.3 & $1,300,500$ & $1,357.700$ & 70 & $05 / 22$ \\
\hline \multirow[t]{2}{*}{ counlt2 batchent } & $63-21-56$ & 153.200 & 7.400 & 98.4 & $3,121,300$ & $3,201,200$ & es & $05 / 27-05 / 22$ \\
\hline & $63-22-55$ & 121.300 & 2,200 & 5.2 & $2,773,400$ & $2,096,500$ & 77 & $05 / 12-05 / 20$ \\
\hline \multirow[t]{2}{*}{ 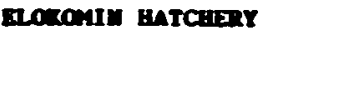 } & $63-22-36$ & 156.200 & 4,000 & 97.7 & $2,755,400$ & $2,915,600$ & 102 & $06 / 01$ \\
\hline & $63-23-17$ & 9,400 & $-0-$ & 100.0 & $-0-$ & 9.400 & 100 & $0, / 01$ \\
\hline \multirow[t]{2}{*}{ EaYs RIVER batcisar } & $63-22-63$ & 64,100 & 800 & $\boldsymbol{9 . 0}$ & $1,145,700$ & $1.210,600$ & $\mathbf{s}$ & $06 / 01-06 / 00$ \\
\hline & $63-23-60$ & 10,200 & $-0-$ & 100.0 & $-0-$ & 10.200 & 93 & estor \\
\hline 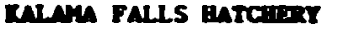 & $63-20-36$ & 175,400 & 3.200 & 2.2 & $3,432,200$ & $3,611,400$ & 103 & $05 / 22-05 / 20$ \\
\hline \multirow[t]{2}{*}{ mascanine antaid } & $07-22-27$ & 18.900 & $\sec$ & 97.5 & 718.60 & 737.400 & $\mathbf{e s}$ & $05 / 10$ \\
\hline & $07-23-32$ & 02.100 & 2,100 & 92.5 & $3,121, \infty 60$ & $3,206,600$ & $\mathbf{e s}$ & $05 / 10$ \\
\hline nickitat antaudar & $63-20-00$ & 130.000 & 2.700 & s... & $2,346,5 \times 1$ & $2,479,200$ & $m$ & os/es \\
\hline \multirow[t]{3}{*}{ LITTLE MITE aArc in } & $05-07-47$ & 183,400 & 4.700 & 97.5 & $6,507,300$ & 6.775 .460 & s4 & es/en - os/es \\
\hline & $05-10-49$ & 52.400 & 1.400 & 92.4 & $1,003,300$ & 1.937 .100 & s4 & $06 / 04-06 / 05$ \\
\hline & $05-00-50$ & 13,300 & $\infty$ & 93.6 & 409.200 & 503,100 & $\boldsymbol{3 4}$ & Cs/Oa - es/os \\
\hline
\end{tabular}


Iable 3.--(Conc Inued)

\begin{tabular}{|c|c|c|c|c|c|c|c|c|}
\hline Rearine Facllity & Tas code & $\begin{array}{l}\text { Targed Fish } \\
\text { Released }\end{array}$ & $\begin{array}{l}\text { Ad Only Fish } \\
\text { Released }\end{array}$ & $\begin{array}{c}\text { I Ias } \\
\text { Rercation }\end{array}$ & $\begin{array}{l}\text { Unarked Fish } \\
\text { Released }\end{array}$ & $\begin{array}{c}\text { Total } \\
\text { Releese }\end{array}$ & F1sh/1b & Release Dates \\
\hline LOWER KNLAMA HATCHERY & $63-22-54$ & 15s, 300 & 6.500 & 96.0 & $2,036,900$ & $2,998,700$ & 98 & $06 / 01-06 / 10$ \\
\hline \multirow[t]{2}{*}{ PRIEST RAPIDS HATCHERY } & $63-21-55$ & 194.600 & 1,500 & 99.3 & $3,793,200$ & $3,989,300$ & 89 & $06 / 23=06 / 24$ \\
\hline & $63-22-61$ & 42.100 & 100 & 99.7 & 787,900 & 830.100 & 67 & $05 / 18$ \\
\hline SEA RESOURCES AATCHERY & $63-22-01$ & 43.300 & 1.100 & 97.6 & 786.800 & 831,200 & 90 & $04 / 16-04 / 29$ \\
\hline \multirow[t]{10}{*}{ SPRIMG CREEK aATcasRY } & $05-07-60$ & 104.700 & 400 & 99.6 & 4.743 .200 & $4,048,300$ & 90 & $03 / 25$ \\
\hline & $05-07-61$ & 76.700 & 800 & 99.0 & $3.217,000$ & $3,193,300$ & 71 & $04 / 15$ \\
\hline & $05-07-42$ & 63.100 & 300 & 99.5 & $3,141,500$ & $3,206,000$ & 65 & os/os \\
\hline & $05-07-43$ & 25,700 & 100 & 99.5 & 123.900 & 149.700 & 75 & $04 / 21-04 / 22$ \\
\hline & $05-07-46$ & 150,500 & 800 & 92.5 & 724,700 & 076,000 & 75 & $04 / 21-04 / 22$ \\
\hline & $05-07-68$ & 28,800 & 100 & 99.5 & $1,345,400$ & 1.376 .300 & 118 & $03 / 25$ \\
\hline & $05-07-49$ & 30,900 & 300 & 99.0 & $1,255,000$ & $1,226,200$ & 71 & $04 / 15$ \\
\hline & $05-07-50$ & 13,700 & $-0-$ & 100.0 & 635,200 & 646,900 & 121 & $03 / 25$ \\
\hline & $05-07-51$ & 15,400 & 100 & 99.5 & 748,000 & 763.500 & 102 & $03 / 25$ \\
\hline & $05-07-52$ & 7,200 & 200 & $97 . \bullet$ & 283,600 & 291,000 & 15 & $08 / 12$ \\
\hline STAYTON POND & $07-23-35$ & 245.500 & 7.300 & 97.0 & $3,649,700$ & $5,902,700$ & 75 & $04 / 27-06 / 15$ \\
\hline \multirow[t]{2}{*}{ WASHOUGAL HATCHERY } & $63-21-48$ & 28,700 & 300 & 99.1 & 483.200 & 512.200 & 35 & $07106-09 / 04$ \\
\hline & $63-22-51$ & 278,000 & 3,100 & 93.9 & $5.228,000$ & $5.509,900$ & 76 & $06 / 30-07 / 06$ \\
\hline \multirow[t]{2}{*}{ UEYCO POND } & $m-03-01$ & 169,500 & 2.700 & $\boldsymbol{9 0 . 4}$ & $3.320,100$ & $3,500,300$ & 90 & $05 / 15-06 / 12$ \\
\hline & a1-03-02 & 64.300 & 600 & 9.0 & $1,200,100$ & $1,273,000$ & $\boldsymbol{s 0}$ & $05 / 15-06 / 12$ \\
\hline Tocai & & $3,466,400$ & 03,200 & & $86.2 \%, 000$ & $09,048,600$ & & \\
\hline
\end{tabular}


Table 4.--Releases of 1981-brood fall chinook salmon fron Columble River facliries in 1902.

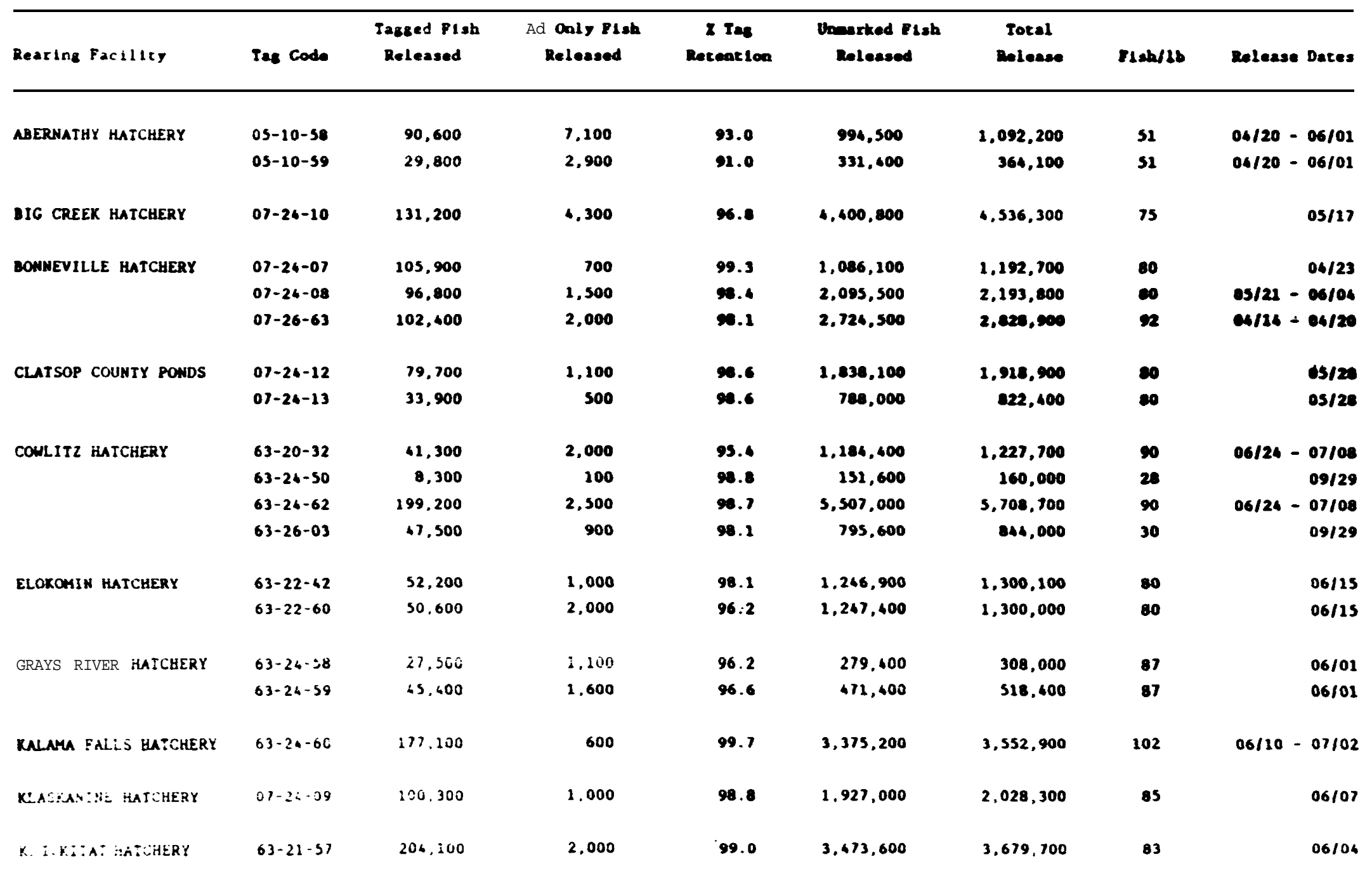


Iable 4.--(Cont/lound)

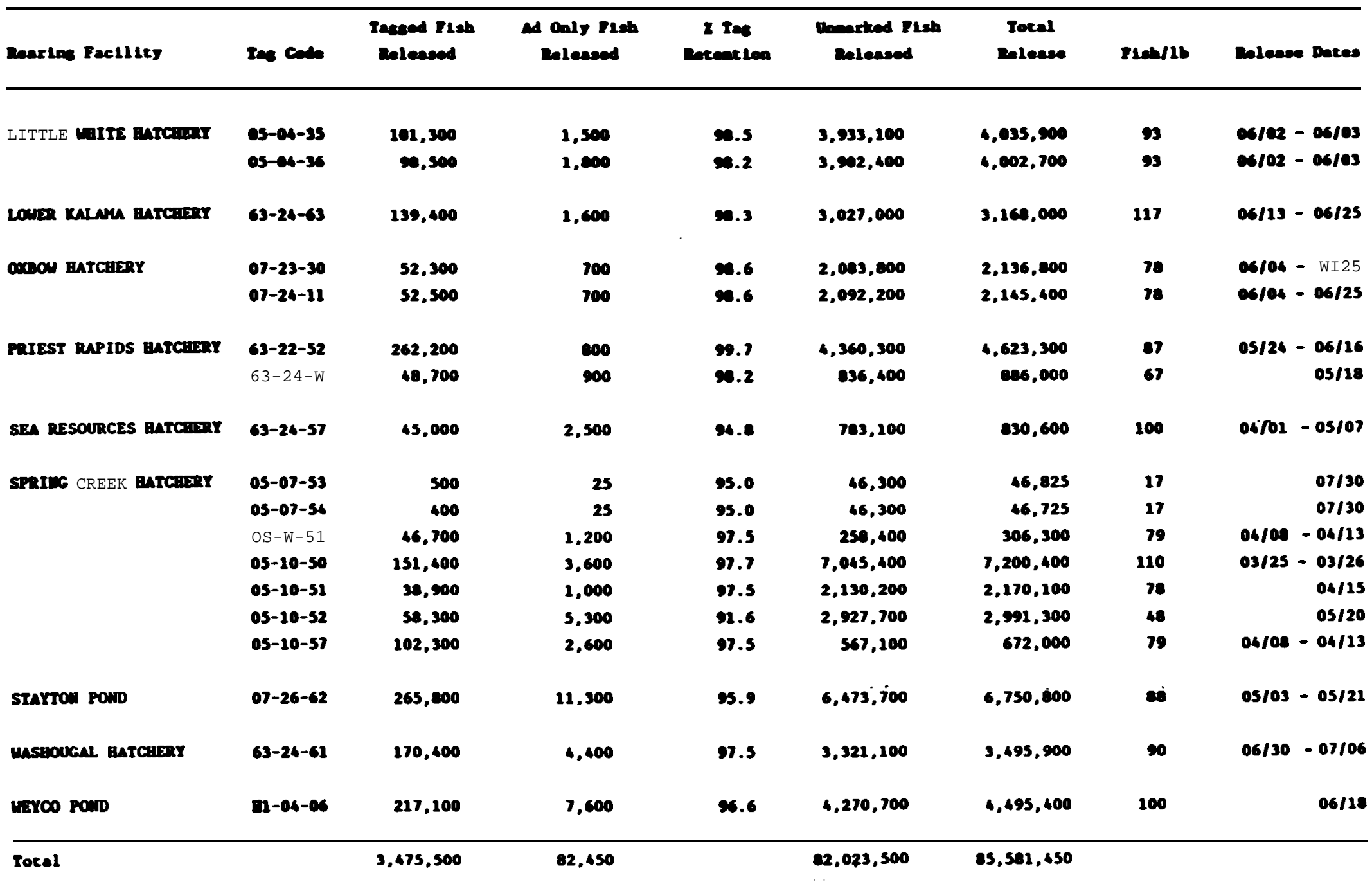




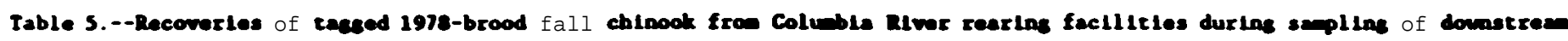
elerants at varlous locations on the Columbla River in 1979 and 1920.

\begin{tabular}{|c|c|c|c|c|c|c|}
\hline & & Taceed & & Decovery & Huber of recoverles & \\
\hline Rearlus facilley & Tas code & releases & Release dates & lecet loan & observedo stinated 2 & Recovery dates \\
\hline
\end{tabular}

ABERMATYY $M-Y$

$05-04-50$

63,400

$04 / 17-05 / 18 / 79$

urez Estuary

Lowa Estung

60
9

$$
\text { 05-04-51 }
$$

48,900

$06 / 17-05 / 18 / 79$

urea estunay

35
3

\begin{tabular}{|c|c|c|c|c|c|c|c|}
\hline BIG Coen antarixy & $07-18-46$ & 224,900 & $05 / 21 / 79$ & $\begin{array}{l}\text { Lovin Estunt } \\
\text { cocen }\end{array}$ & \begin{tabular}{c}
41 \\
3 \\
\hdashline 4
\end{tabular} & $\begin{array}{l}0 \\
0\end{array}$ & $\begin{array}{l}05 / 24-07 / 17 / 79 \\
06 / 20-07 / 04 / 79\end{array}$ \\
\hline BIG wite Pow & $05-04-43$ & 141,400 & $05 / 21 / 79$ & $\begin{array}{l}\text { Uste estungy } \\
\text { Lowe estungy } \\
\text { coter }\end{array}$ & $\begin{array}{r}56 \\
13 \\
2 \\
71\end{array}$ & $\begin{array}{r}\mathbf{0 2} \\
\mathbf{0} \\
0\end{array}$ & $\begin{array}{l}06 / 24-06 / 00 / 79 \\
06 / 04-07 / 02 / 79 \\
06 / 28 / 79\end{array}$ \\
\hline BOMEVILLE BATCHIXYY & $07-18-42$ & 287.900 & $05 / 01-05 / 29 / 79$ & $\begin{array}{l}\text { UPPE ESTUARY } \\
\text { Lown ESTwary } \\
\text { ocen }\end{array}$ & $\begin{array}{r}499 \\
48 \\
4 \\
551\end{array}$ & $\begin{array}{r}590 \\
0 \\
0\end{array}$ & $\begin{array}{l}05 / 04-07 / 13 / 79 \\
05 / 18-07 / 04 / 79 \\
06 / 28-07 / 04 / 79\end{array}$ \\
\hline
\end{tabular}

69

46

$04 / 18-06 / 22 / 79$ $05 / 17-06 / 18 / 79$

$06 / 10-06 / 12 / 79$ $05 / 18-06 / 15 / 79$

3 
Table S. -- (Cont Inued)

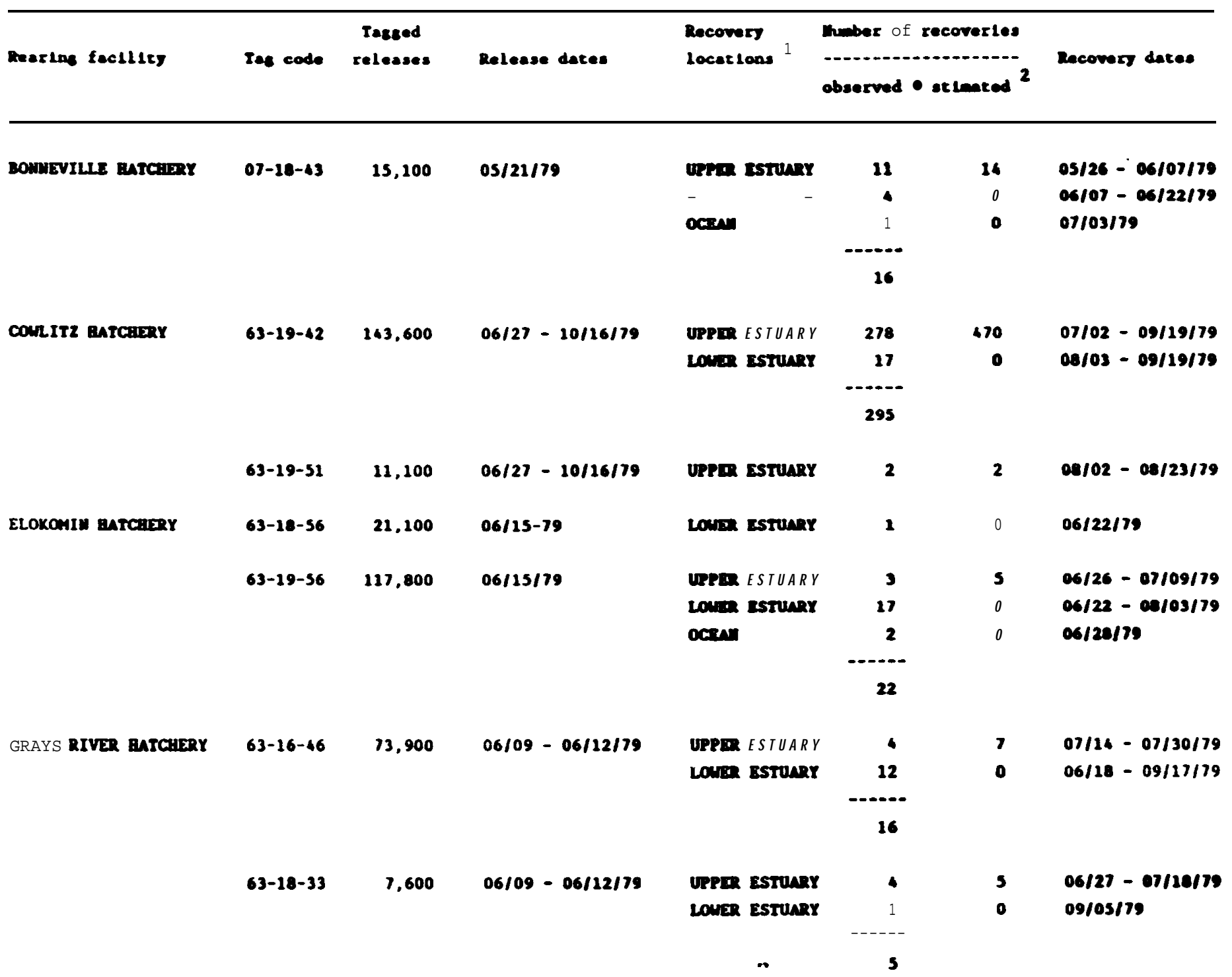


Table S.--(Cone 1ound)

\begin{tabular}{|c|c|c|c|c|c|c|}
\hline & & Taceed & & Recovery & Whaber of recoveries & \\
\hline Rearine factlley & Tas code & releases & Release dates & locations ${ }^{1}$ & observede stinted 2 & Recovery dates \\
\hline
\end{tabular}

$68,100 \quad 06 / 09=06 / 12 / 79$

\begin{tabular}{|c|c|c|c|c|}
\hline Grays RIVER Batcueny & $63-19-37$ & 68,100 & $06 / 09=06 / 12 / 79$ & 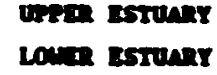 \\
\hline
\end{tabular}

$5 \quad 06 / 27-07 / 23179$

11

\begin{tabular}{|c|c|c|c|c|c|c|c|}
\hline raLAMA FaLLS aATCBERY & $63-19-57$ & 214.500 & $06 / 22 \quad 07 / 13 / 79$ & 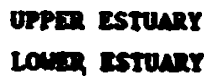 & $\begin{array}{r}2.229 \\
.\end{array}$ & $\begin{array}{r}3.005 \\
0\end{array}$ & $\begin{array}{l}06 / 24-09 / 14 / 79 \\
07 / 13-09 / 18 / 79\end{array}$ \\
\hline DLASTAMINE BATCaBgy & $07-18-45$ & 264.100 & $05 / 29 / 79$ & $\begin{array}{l}\text { LOVIX ESTUARY } \\
\text { OCEAN }\end{array}$ & $\begin{array}{r}25 \\
7 \\
32\end{array}$ & $\begin{array}{l}0 \\
0\end{array}$ & $\begin{array}{l}06 / 04-07 / 04 / 79 \\
06 / 28 / 79\end{array}$ \\
\hline ILICRITAT BaTCaEAY & $63-19-49$ & 225,400 & $05 / 14-06 / 13 / 79$ & 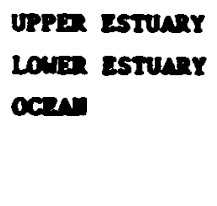 & $\begin{array}{r}224 \\
22 \\
5 \\
251\end{array}$ & $\begin{array}{r}278 \\
0 \\
0\end{array}$ & $\begin{array}{l}05 / 27=07 / 18 / 79 \\
05 / 29-07 / 20 / 79 \\
06 / 20-07 / 03 / 79\end{array}$ \\
\hline
\end{tabular}

LITTLE wrte ancuma $05-04-40 \quad 177,000 \quad 06 / 22 / 79$

\begin{tabular}{|c|c|c|c|}
\hline Ures estwax & 25n & 391 & $06 / 27-08 / 16 / 79$ \\
\hline Loute estungr & 12 & 0 & $07 / 02-09 / 14 / 79$ \\
\hline ocren & 1 & 0 & $08 / 09 / 79$ \\
\hline & 267 & & \\
\hline
\end{tabular}


Table 3.--(Coatinuod)

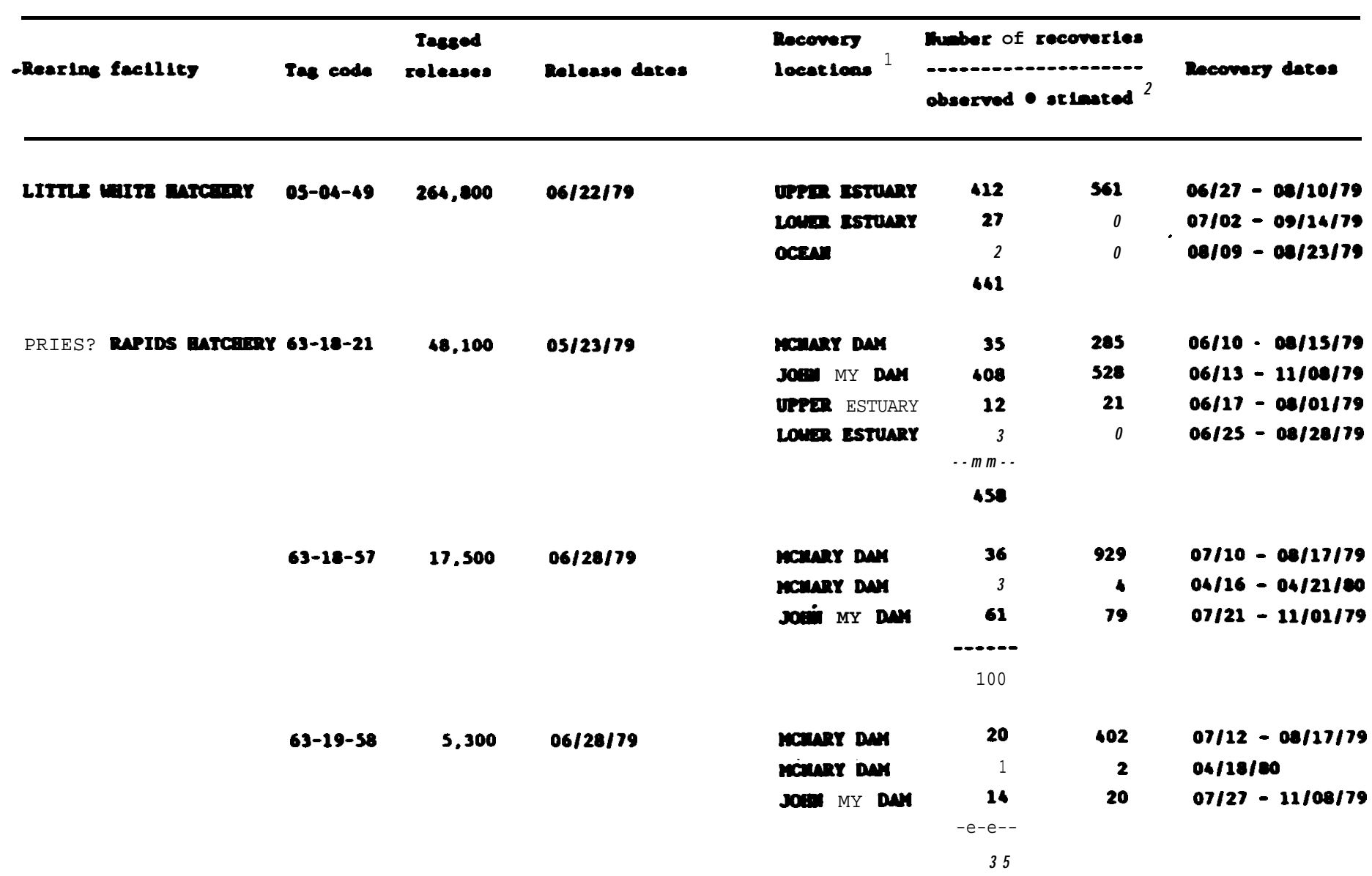


Table 5. --(Coat lound)

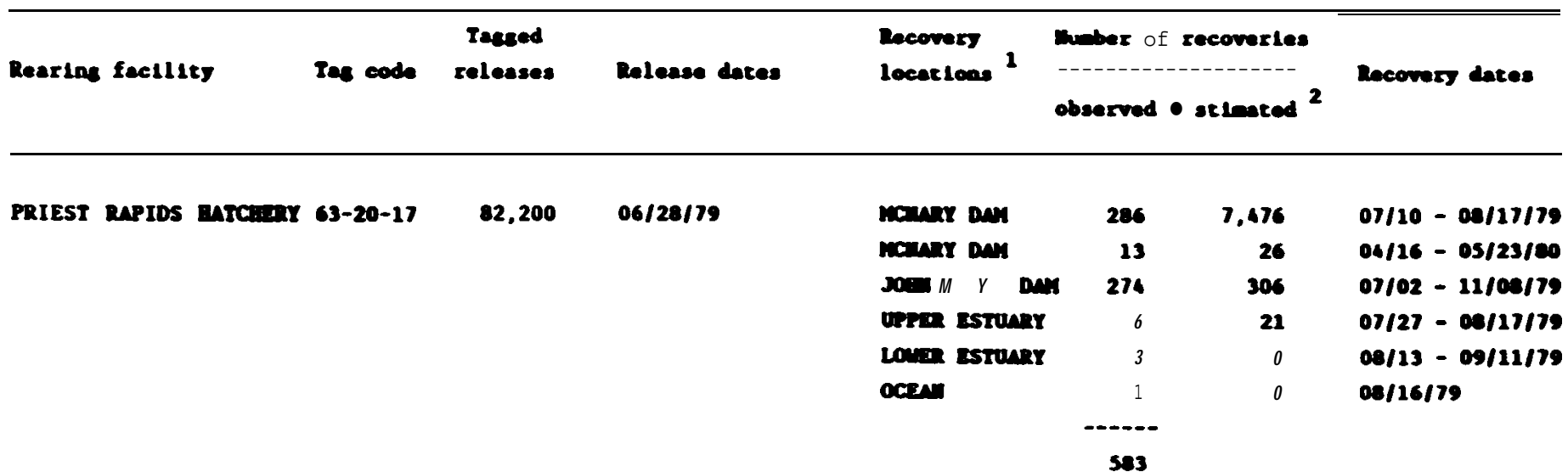

SPEEral aAtaiar $\quad 63-19-20 \quad 31.700 \quad 09 / 05 / 7$

ores estone

$-0-$

$0 \quad-0-$

SPEDYYI BATCiter

$63-19-20$

$\$ 1.700$

Lora estung

$\begin{array}{rrr}18 & 123 & 09 / 10-09 / 26 / 79 \\ 9 & 0 & 09 / 09-09 / 27 / 79\end{array}$

27

$63-19-50 \quad 104.500 \quad 07 / 19 / 79$

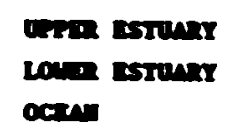

030

12

1.140

$07 / 20-09 / 24 / 79$

corin

$00 / 21-00 / 27 / 79$

os/osits

eas

Spruc arex entcing 05-04-33 140,900 05/18/78

P I P - s

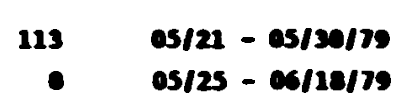


Table 5.---(contimed)

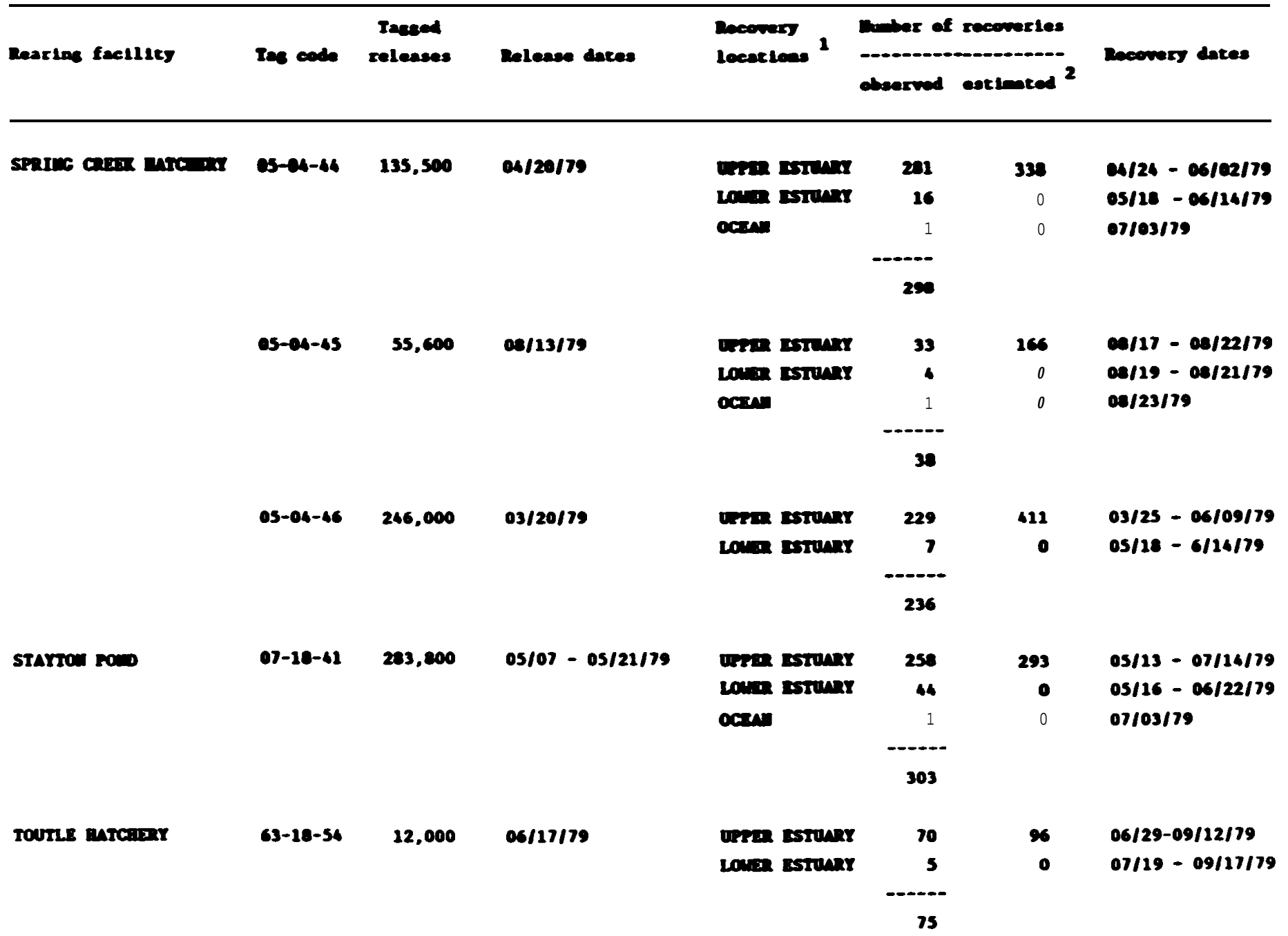


Table S.--(Cont Lound)

\begin{tabular}{|c|c|c|c|c|c|c|}
\hline & & Taceed & & Decovery & Muber of recovertes & \\
\hline Rearlos facllity & Ines code & releases & Release dates & locatlone 1 & observed estinated 2 & Ineovers dates \\
\hline
\end{tabular}

TOUTL Burcing $\quad 63-19-41 \quad 132,100 \quad 06 / 17 / 79 \quad 06 / 12-09 / 19 / 79$

Lave estruax $25 \quad 07 / 11-09 / 14 / 79$

coril $3000 / 09-00 / 15 / 79$

022

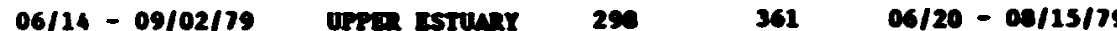

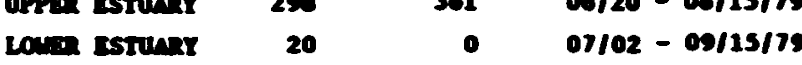

OCTII $2000120-07104 / 79$

$\omega$

63-19-46 154.500 06/14-09/02/79 uren EsTunx s09 $730 \quad 05 / 19-00 / 29 / 79$

Low esturix $23 \quad 07 / 02-05 / 24 / 79$

$\mathbf{6 1 2}$

UEYCO POW

63-19-39 $\quad 92.400 \quad 06 / 05 / 79$

urien essunx

coun sown

octer

$07 / 17-00 / 11 / 78$

$06 / 14$ - 05/17/7s

$06 / 28 / 79$

14

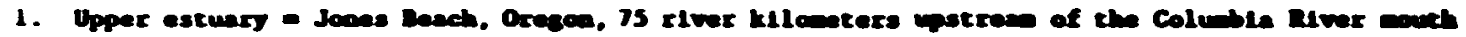

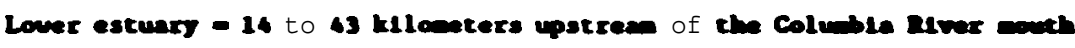

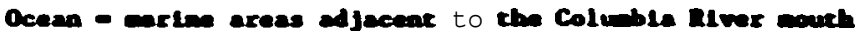

2. Oaly recoverles at Jowes Beach were standardized for fishing offort. 


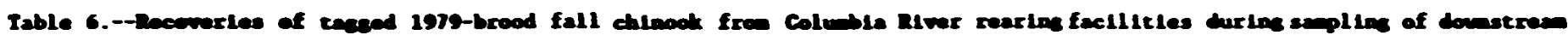
elerante st varlons lecations on the Colwable niver in 1500 and 151.

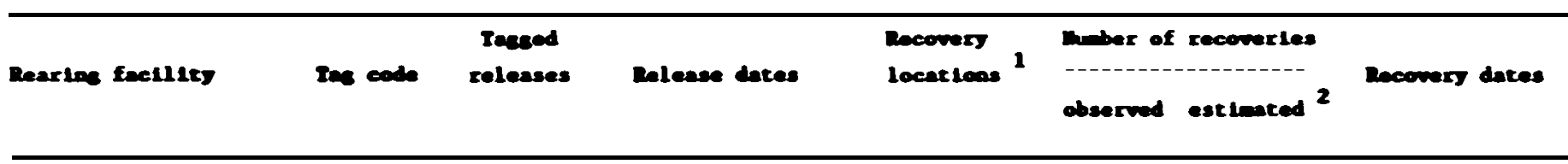

Angoming ente ax

05-es-46

35.200

$04 / 09-05 / 16 / 50$

use estung

10

32

$04 / 05-05 / 19 / 100$

corse estury

1
22

$05-06-46 \quad 112,500$

$04 / 09=05 / 14 / 50$

UThe Estunar

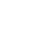

cong estung

42

2

$64 \quad 06 / 09-07 / 00 / 50$

- $05 / 22-05 / 23 / 80$

\begin{tabular}{|c|c|c|c|c|c|c|c|}
\hline 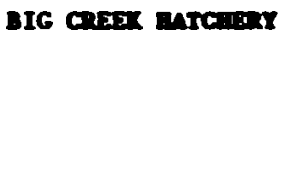 & $07-21-60$ & 143,400 & $05 / 13 / 60$ & $\begin{array}{l}\text { Lowim Estung } \\
\text { ocen }\end{array}$ & $\begin{array}{c}5 \\
4 \\
9\end{array}$ & $\begin{array}{l}\mathbf{0} \\
\mathbf{0}\end{array}$ & $\begin{array}{l}05 / 20-07 / 18 / 100 \\
06 / 17-07 / 13 / 20\end{array}$ \\
\hline sombertus enrain & $07-21-57$ & 121,100 & $05 / 20-05 / 28 / 60$ & 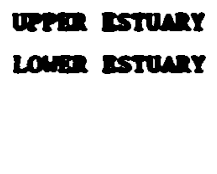 & $\begin{array}{c}56 \\
9 \\
65\end{array}$ & $\begin{array}{r}110 \\
0\end{array}$ & $\begin{array}{l}05 / 27-07 / 02 / 100 \\
06 / 16-06 / 24 / 100\end{array}$ \\
\hline \multirow[t]{3}{*}{ conlit2 anteigar } & $63-21-37$ & 20.700 & $03 / 21-04 / 01 / 81$ & urper estungy & 17 & 40 & $03 / 25-04 / 26 / 81$ \\
\hline & $63-21-54$ & 244,300 & $06 / 03-07 / 11 / 20$ & 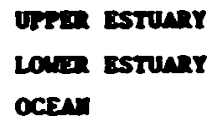 & $\begin{array}{r}66 \\
36 \\
2\end{array}$ & $\begin{array}{r}115 \\
0 \\
0\end{array}$ & $\begin{array}{l}07 / 03-09 / 17 / 80 \\
06 / 18-08 / 19 / 80 \\
07 / 21 / 80\end{array}$ \\
\hline & & & & & 204 & & \\
\hline
\end{tabular}


Table 6. --(Coarinoud)

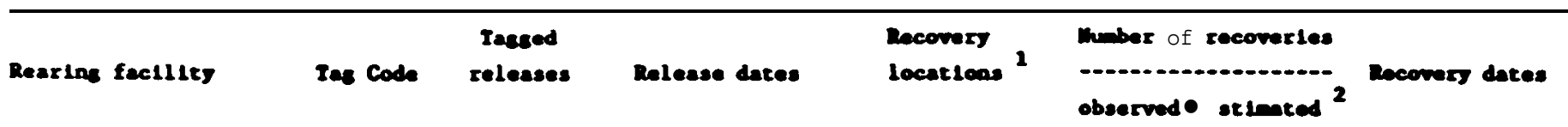

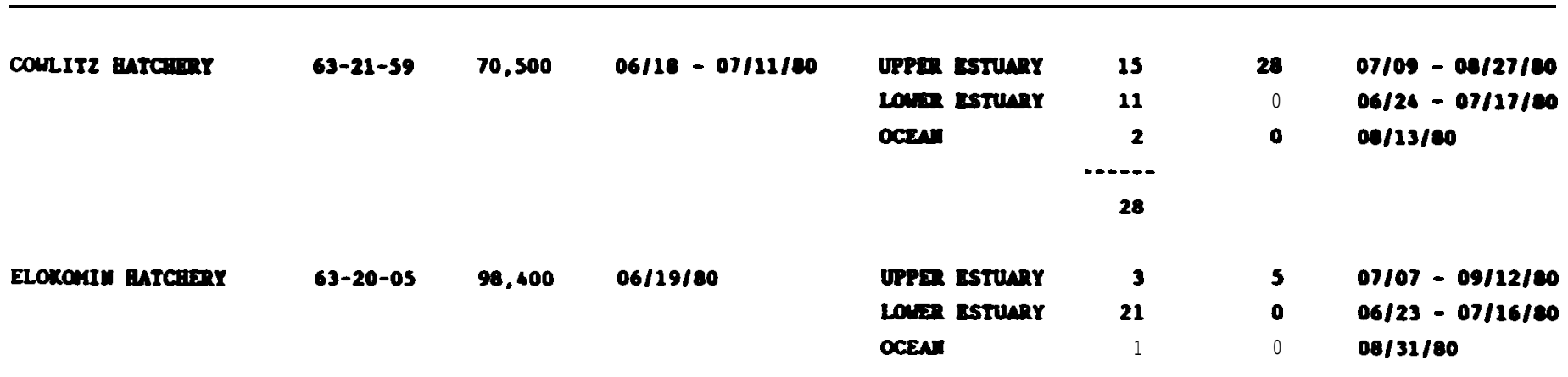

$\omega$

\begin{tabular}{|c|c|c|c|c|c|c|c|}
\hline GRAYS RIVER BATCAIZYY & $63-20-43$ & 37,500 & $06 / 01-06 / 24 / 20$ & Loving estuary & 3 & o & $06 / 22-07 / 14 / 00$ \\
\hline XALAMA Falls Batchiary & 63-21-05 & 100,400 & $06 / 13-06 / 24 / 20$ & $\begin{array}{l}\text { UPPER ESTUARY } \\
\text { UPPER ESTUARY } \\
\text { LOWR ESTUARY } \\
\text { OCEAI }\end{array}$ & $\begin{array}{r}\mathbf{1 6 3} \\
1 \\
\mathbf{1 7} \\
1 \\
\mathbf{1 8 2}\end{array}$ & $\begin{array}{r}243 \\
1 \\
0 \\
0\end{array}$ & $\begin{array}{l}06 / 16-09 / 08 / 80 \\
04 / 13 / 21 \\
06 / 19-07 / 29 / 80 \\
00 / 13 / 80\end{array}$ \\
\hline XLASKANIME BATCBERY & $07-21-61$ & 66,300 & $06 / 04 / 80$ & OCENM & 1 & - & $06 / 17 / 80$ \\
\hline LLICRITAT BATCHERY & $63-19-47$ & 156,100 & $05 / 27 / 20$ & $\begin{array}{l}\text { Uprea Estuney } \\
\text { Love Estunery }\end{array}$ & $\frac{64}{12}$ & $\begin{array}{r}106 \\
0\end{array}$ & $\begin{array}{l}06 / 02-07 / 30 / 20 \\
06 / 16-07 / 11 / 20\end{array}$ \\
\hline
\end{tabular}


Table 6.--(Coat Lound)

\begin{tabular}{|c|c|c|c|c|c|c|c|}
\hline Rearlof facllity & Tas code & $\begin{array}{l}\text { Taused } \\
\text { releases }\end{array}$ & Release dates & $\begin{array}{l}\text { Becovery } \\
\text { lecations }\end{array}$ & $\begin{array}{l}\text { Hober } 0 \\
\text { observed }\end{array}$ & Ineded 2 & Racorers dates \\
\hline LEUIS RIVER aATcienY & $63-21-60$ & 103,700 & $07 / 15 / 20$ & $\begin{array}{l}\text { UPPa estunar } \\
\text { upar estunay } \\
\text { ocen }\end{array}$ & $\begin{array}{r}197 \\
2 \\
3 \\
202\end{array}$ & $\begin{array}{r}301 \\
5 \\
0\end{array}$ & $\begin{array}{l}07 / 10-09 / 19 / 80 \\
03 / 19-03 / 30 / 81 \\
07 / 24-08 / 16 / 80\end{array}$ \\
\hline LITTLE waIte BATcason & $05-06-43$ & 162.600 & $06 / 10 / 80$ & $\begin{array}{l}\text { UPPER ESTUARY } \\
\text { Lowa EsTunay }\end{array}$ & $\begin{array}{c}94 \\
41 \\
135\end{array}$ & $\begin{array}{r}116 \\
0\end{array}$ & $\begin{array}{l}06 / 140-07 / 16 / 80 \\
06 / 10-07 / 14 / 80\end{array}$ \\
\hline LOUER rulay Batcisary & $63-20-06$ & 144,500 & $06 / 10 / 80$ & 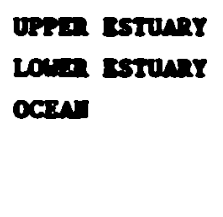 & $\begin{array}{r}209 \\
12 \\
1 \\
---- \\
222\end{array}$ & $\begin{array}{r}289 \\
0 \\
0\end{array}$ & $\begin{array}{l}06 / 07-09 / 09 / 20 \\
06 / 10-00 / 25 / 20 \\
00 / 13 / 20\end{array}$ \\
\hline OxBou Batchisi & $07-21-62$ & 49,400 & $05 / 27 / 80$ & 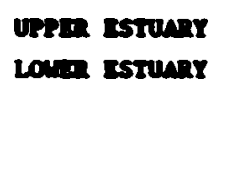 & $\begin{array}{c}21 \\
6 \\
27\end{array}$ & $\begin{array}{r}39 \\
0\end{array}$ & $\begin{array}{l}06 / 02-06 / 26 / 20 \\
06 / 16-07 / 02 / 20\end{array}$ \\
\hline & $07-21-63$ & 51.900 & $05 / 27 / 80$ & 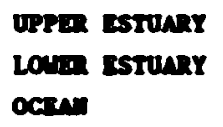 & $\begin{array}{r}20 \\
3 \\
1\end{array}$ & $\begin{array}{r}39 \\
0 \\
0\end{array}$ & $\begin{array}{l}06 / 01-07 / 09 / 80 \\
07 / 20-07 / 15 / 80 \\
06 / 26-06 / 26 / 80\end{array}$ \\
\hline
\end{tabular}


Table 6. --(Coar lound)

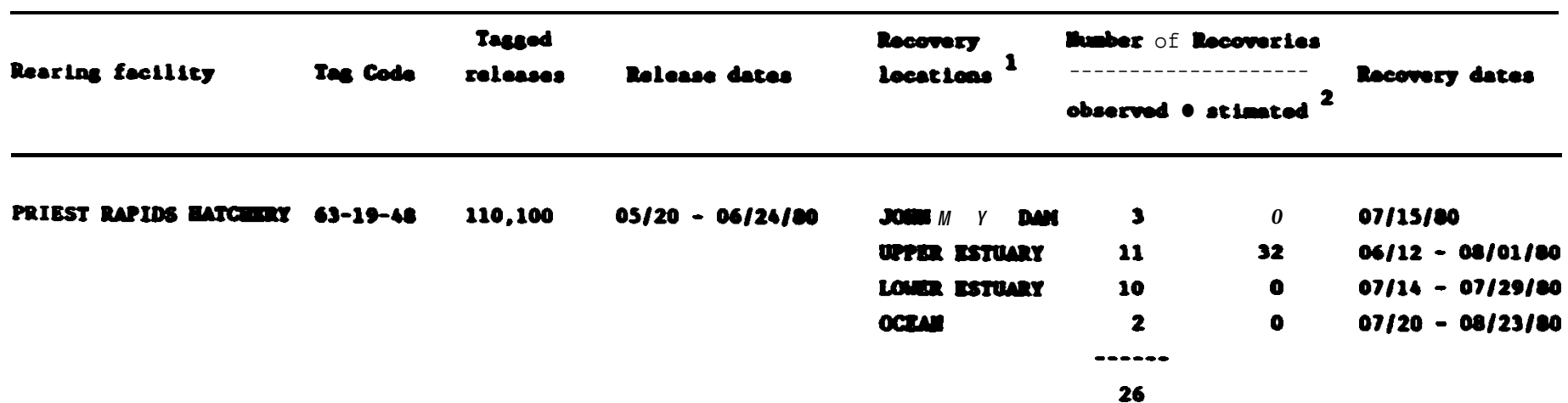

$10,400 \quad 05 / 28 / 60$

$-0-$

$-0-$

vere estung Lowd Estunt

123
4
127

05-06-40 75.200 04/10/80

05-06- 61

60,500

05-06- 42
23.100
$05 / 09 / 80$

$08 / 07 / 80$

$03 / 10 / 20$$$
05110180
$$

(2)

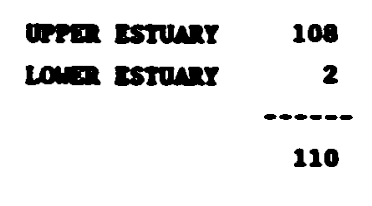

uren estung Lora estuar

$\begin{array}{r}55 \\ 9 \\ \hdashline-\end{array}$

64

urea estung
cosen

9
3
12

\section{$27603 / 24-06 / 10 / 20$}

- $05 / 16-05 / 23 / 20$
$17304 / 14-05 / 17 / 60$
- $05 / 20-05 / 21 / 80$

o1 $05 / 11-06 / 12 / 80$ $05 / 20-05 / 23 / 80$ 
Table 6.--(Coatlound)

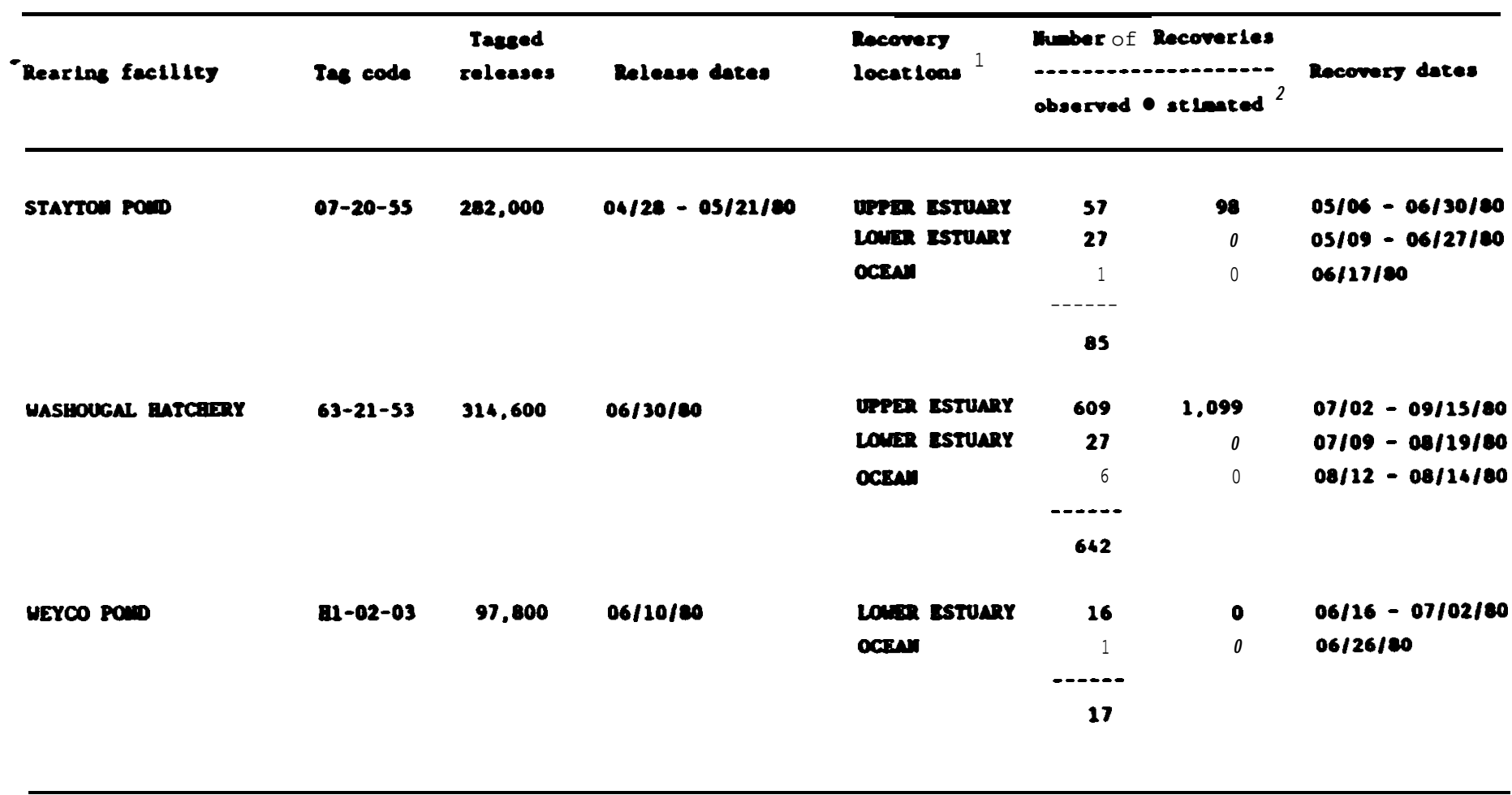

1. Upper - stuary - Jonaileach, Oregon, 75 ziver klloseters upetrean of the Columbla River nouch Loverestuary = 14 tot3 kllomers upstrean of the Coluble River mouth

Ocean - earlme areas adjecent to the Columbla River eouth

2. Oaly recoverles at $\mathrm{J} O \mathrm{~N} a \mathrm{~S}$ Beach were standardized for flehing $\bullet$ ffort. 


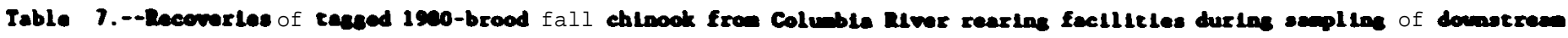
algrancs - t varlous locations on the Columble alver in 1811.

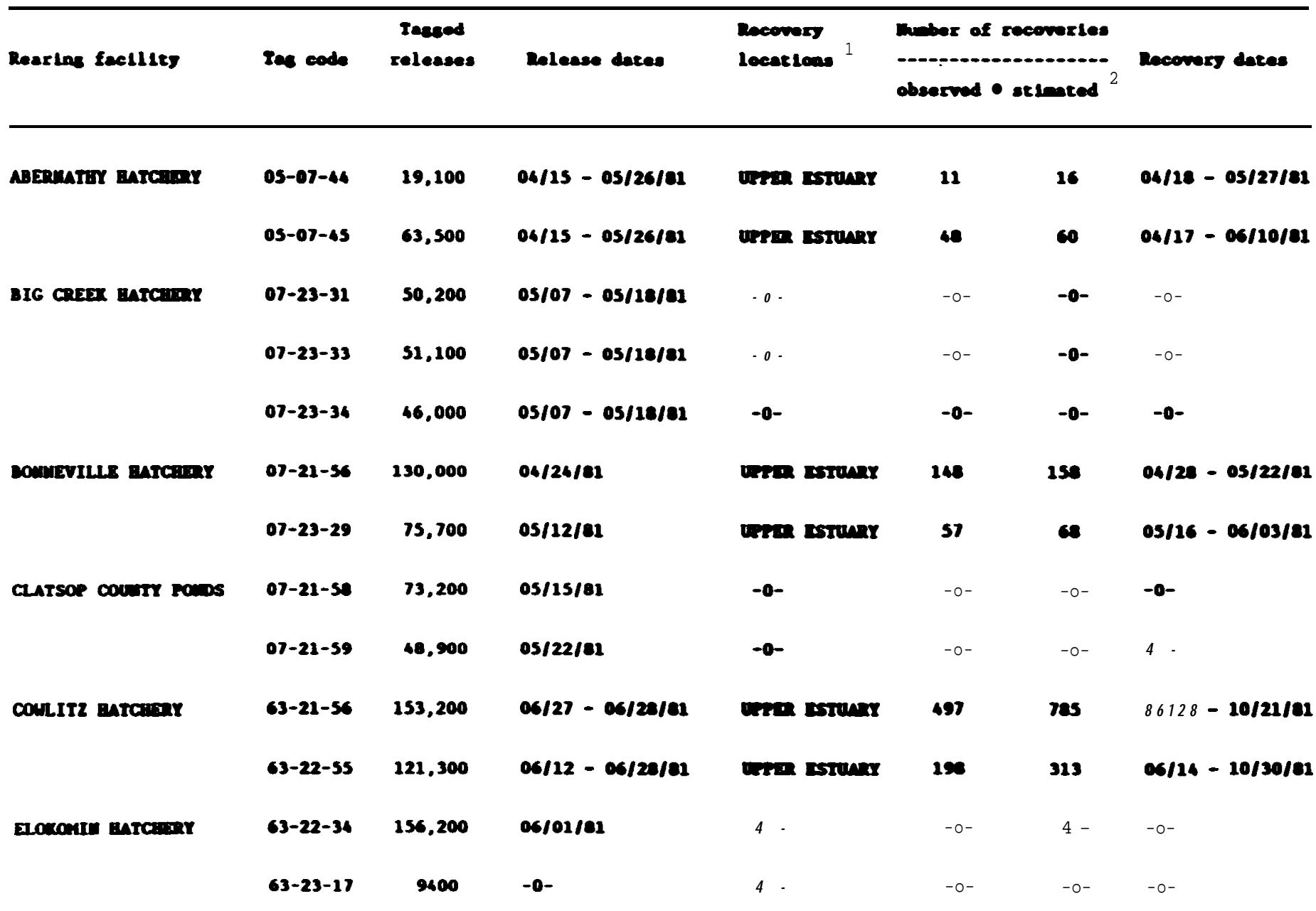


Table 7. --(cosat Lond)

\begin{tabular}{|c|c|c|c|c|c|c|c|}
\hline \multirow[b]{2}{*}{ Dearling faclliey } & \multirow[b]{2}{*}{ Ins Code } & \multirow{2}{*}{$\begin{array}{l}\text { Tacsed } \\
\text { releases }\end{array}$} & \multirow[b]{2}{*}{ Dalease dates } & \multirow{2}{*}{$\begin{array}{l}\text { Ineovery } \\
\text { locentlons }\end{array}$} & \multicolumn{2}{|c|}{ Wuber of Mecoveries } & \multirow[b]{2}{*}{ Docowery dates } \\
\hline & & & & & obeerved & eatimated 2 & \\
\hline \multirow[t]{2}{*}{ Gays RIva mare ix } & $63-22-63$ & 64,100 & $06 / 02-06 / 00 / 81$ & $-0-$ & $-0-$ & $-0-$ & $-0-$ \\
\hline & $63-23-60$ & 10.200 & $06 / 02 / 82$ & $-0-$ & $-0-$ & $-0-$ & $-0-$ \\
\hline ralaM FALLS Batcient & $63-20-36$ & 175.400 & $05 / 22-05 / 20 / 01$ & urrar estuner & 175 & 203 & $05 / 22-07 / 24 / e 1$ \\
\hline \multirow[t]{2}{*}{ rLASEMIME aArCiLat } & $07-22-27$ & 18,900 & $05 / 18 / 81$ & dere estunar & 1 & 2 & $05 / 15 / 81$ \\
\hline & $07-23-32$ & 02,100 & $05 / 18 / 82$ & $-0-$ & $-0-$ & $-0-$ & \\
\hline ILICUITat anciear & $63-20-08$ & 130,000 & 06/05/e1 & ures estuary & 30 & 42 & $06 / 10-08 / 10 / 81$ \\
\hline \multirow[t]{3}{*}{ LITTL wire entoiny } & $05-07-67$ & 183,400 & $06 / 04-06 / 05 / 01$ & URPE Estuary & 117 & 130 & $06 / 08-07 / 05 / 81$ \\
\hline & $05-05-49$ & 52.400 & $06 / 04-06 / 05 / 01$ & uren exwax & 43 & 46 & $06 / 09-07 / 02 / 81$ \\
\hline & $05-02-50$ & 13,300 & $06 / 04-06 / 05 / 01$ & ure estunx & 4 & 5 & $06 / 09-06 / 13 / 81$ \\
\hline Lowze rulare Entoing & $63-22-56$ & 155,300 & $06 / 01-06 / 20 / 01$ & gere estung & 175 & 209 & $06 / 03-08 / 18 / 81$ \\
\hline \multirow[t]{2}{*}{ PRIEST RAPIDS matciax } & $63-21-55$ & 194,600 & $06 / 23-06 / 24 / 101$ & uren esruax & 33 & 142 & $07 / 09-09 / 10 / 01$ \\
\hline & $63-22-61$ & 42,100 & os/1e/e1 & Dren Estunx & 13 & 35 & $06 / 18-07 / 21 / 81$ \\
\hline
\end{tabular}


Table $7 .--(\operatorname{coat} L$ rand $)$

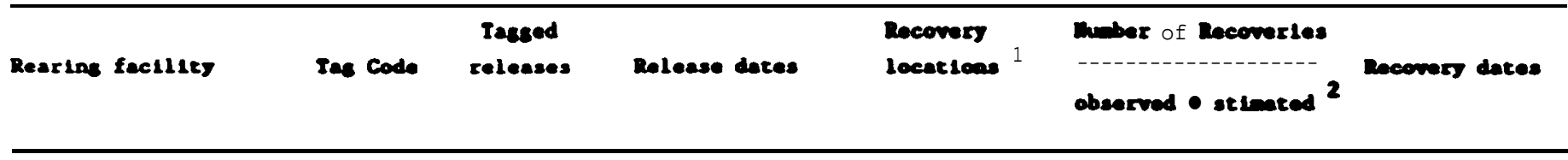

\begin{tabular}{|c|c|c|c|c|c|c|c|}
\hline SEA RESOURCEs autcany & $63-22-01$ & 43,300 & $04 / 16-04 / 29 / 81$ & $-0-$ & $-0-$ & $-0-$ & $-0-$ \\
\hline \multirow{10}{*}{ SPRIMG CREAX hitami } & $05-07-40$ & 104.700 & $03 / 25 / 12$ & Urex exruary & 63 & 100 & $03 / 30-05 / 21 / 101$ \\
\hline & $05-07-41$ & 76.700 & $04 / 15 / 42$ & uera estuary & 70 & 24 & $06 / 19-05 / 19 / 01$ \\
\hline & $05-07-42$ & 63.100 & $05 / 05 / 81$ & Uepe estunix & 105 & 109 & $05 / 09-05 / 30 / 01$ \\
\hline & $05-07-43$ & 25.700 & $04 / 21=04 / 22 / 81$ & urpen estuany & 10 & 13 & $05 / 05-06 / 11 / 01$ \\
\hline & $05-07-46$ & 150,500 & $04 / 22-04 / 22 / 01$ & $\operatorname{arna}$ extuax & 56 & 20 & $05 / 03-06 / 15 / 181$ \\
\hline & $05-07-10$ & 28.800 & $03 / 25 / 82$ & unen estuxy & 12 & 24 & $03 / 30-05 / 20 / 81$ \\
\hline & $05-07-69$ & 30,900 & $04 / 15 / 01$ & upen estuner & 35 & 40 & $04 / 21-05 / 17 / 01$ \\
\hline & $05-07-50$ & 13,700 & $03 / 25 / 81$ & use Erext & 9 & 13 & $03 / 30-05 / 04 / 81$ \\
\hline & $05-07-51$ & 15,400 & $03 / 25 / 01$ & uma estuxi & 8 & 12 & $04 / 03-05 / 06 / 02$ \\
\hline & $05-07-52$ & 7.200 & $08 / 12 / 81$ & $4-$ & $-0-$ & $-0-$ & $-0-$ \\
\hline STAYTOW POED & $07-23-35$ & 265.500 & $04 / 27-06 / 15 / 01$ & - & 169 & 195 & $05 / 06-07 / 02 / 01$ \\
\hline \multirow[t]{2}{*}{ UASBOUGal BATcBisay } & $63-21-40$ & 28.700 & $07 / 06-09 / 04 / 02$ & Gera strux & 19 & so & $07 / 12-10 / 10 / 81$ \\
\hline & $63-22-51$ & 278.800 & $06 / 30-07 / 06 / 01$ & Uere Estuary & 417 & $\infty$ & $06 / 30-09 / 03 / 61$ \\
\hline
\end{tabular}


Table 7.--(Continand)

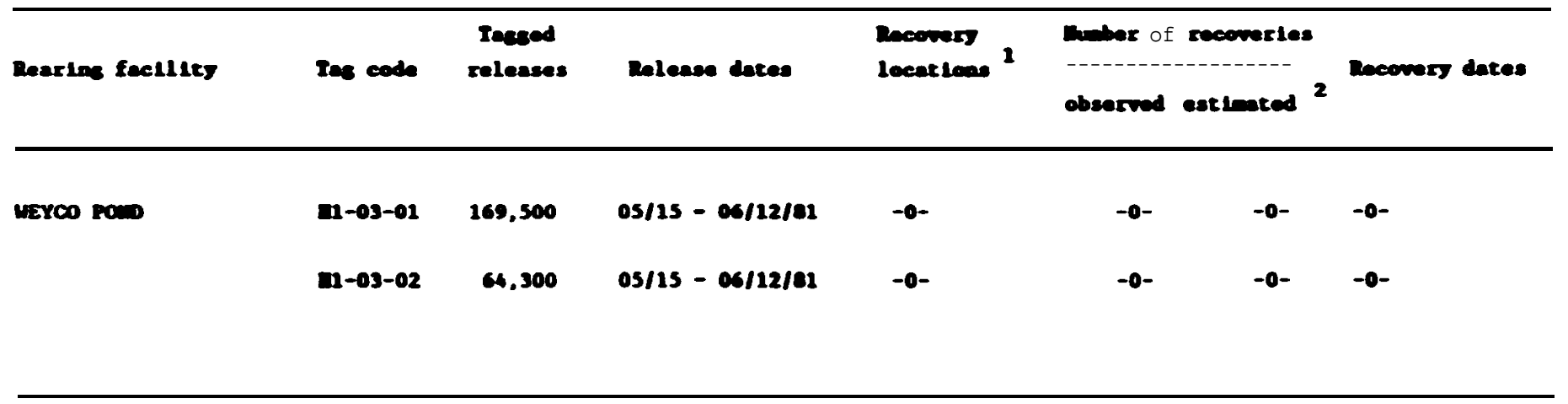

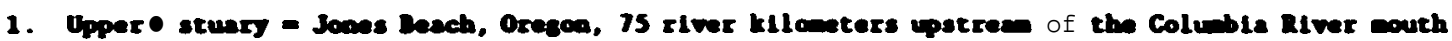

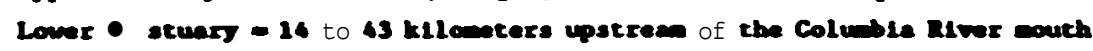

dean = arine areas adjecent to the Columbla River mouth

2. Standardleed for fishine effort at Jones Deach 


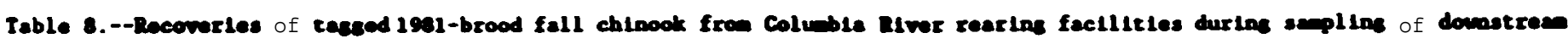
derants at varlous locations on the Colunbla River in 1922.

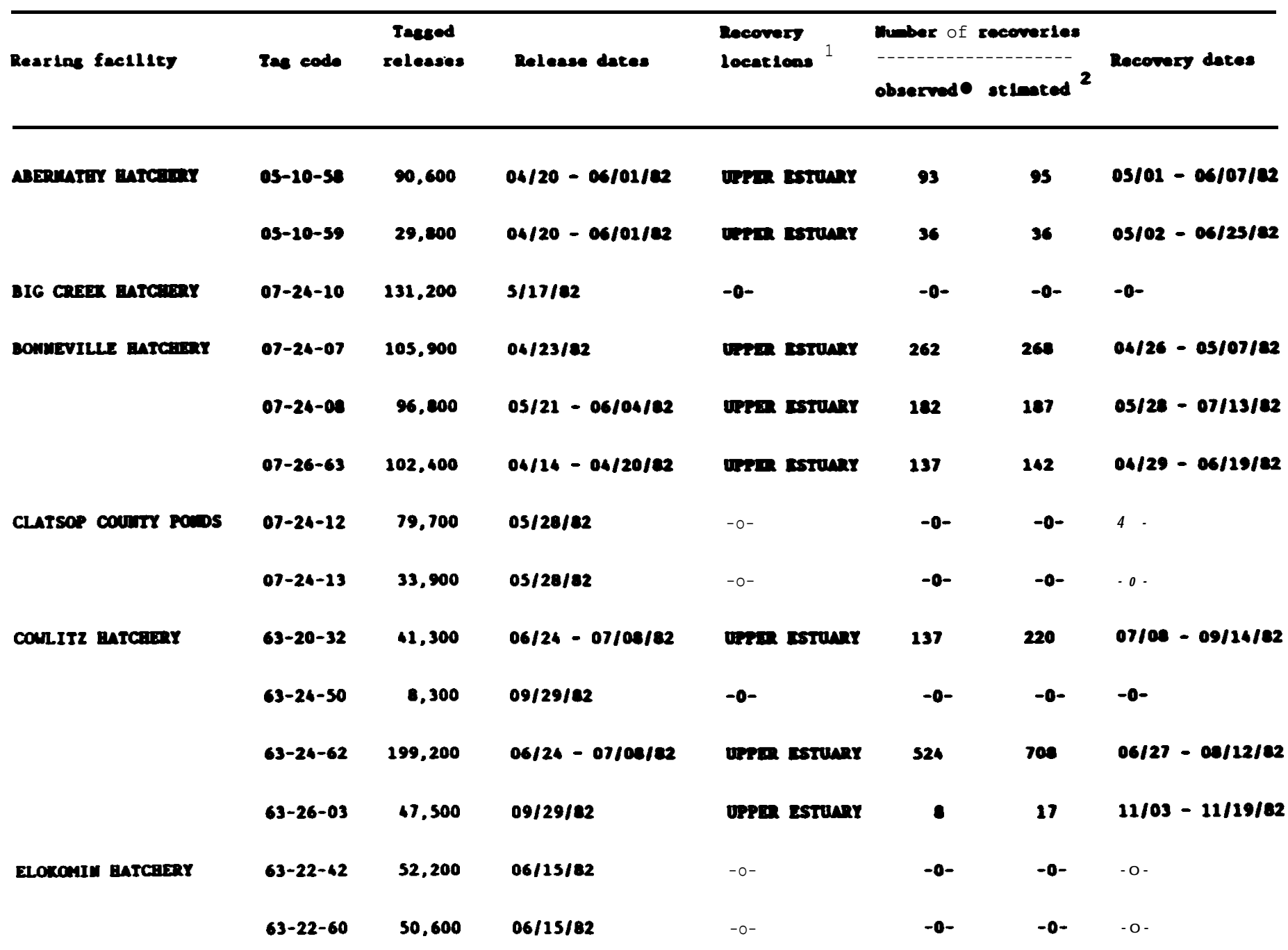


Iable 8. - (Cont Imuad)

\begin{tabular}{|c|c|c|c|c|c|c|c|}
\hline Rearing fecllity & Ias code & $\begin{array}{l}\text { Tacesed } \\
\text { releases }\end{array}$ & Releage dates & $\begin{array}{l}\text { Recovery } \\
\text { lecations }\end{array}$ & \multicolumn{2}{|c|}{ Muber of recoveries } & 2 Recovery dates \\
\hline GRAYs RIVIR Barcion & $63-26-50$ & 27.500 & $06 / 01 / 82$ & $-0-$ & $-0-$ & $-0-$ & $-0-$ \\
\hline ThLam Palls antcianY & $63-20-60$ & 177.100 & $06 / 10-07 / 02 / 82$ & ORPER Estuany & 185 & 266 & $06 / 14-08 / 30 / 82$ \\
\hline ILASTAUINE BATCMERY & $07-24-09$ & 100,300 & $06 / 07 / 82$ & $-0-$ & $-0-$ & $-0-$ & $-0-$ \\
\hline NLICUItat Batcasix & $63-21-57$ & 206.100 & $06 / 04 / 82$ & UPRER Estuary & 214 & 228 & $06 / 03-07 / 17 / 82$ \\
\hline LITTLE Martr aArciser & $05-04-36$ & 98,500 & $06 / 02-06 / 03 / 22$ & URPE Estuna & 146 & 147 & $06 / 06-06 / 15 / 82$ \\
\hline LOWER ralayn EATCaeny & $63-24-63$ & 139,400 & $06 / 13-06 / 25 / 22$ & UPPE ESTUNRY & 193 & 231 & $06 / 14-08 / 27 / 82$ \\
\hline \multirow[t]{2}{*}{ axBow Butcaen } & $07-23-30$ & 52,300 & $06 / 04-06 / 25 / 82$ & UPPER ESTUARY & 45 & 51 & $06 / 21-07 / 01 / 82$ \\
\hline & $07-24-11$ & 52,500 & $06 / 04 / 8-06 / 25 / 82$ & URPE EstuanY & 47 & 53 & $06 / 07-07 / 28 / 82$ \\
\hline \multirow[t]{2}{*}{ PRIEST RAPIDS BArcatiar } & $63-22-52$ & 262.200 & $05 / 24-06 / 16 / 22$ & UPPE EstuARY & 93 & 190 & $06 / 23-07 / 26 / 82$ \\
\hline & $63-26-56$ & 48,700 & $05 / 18 / 82$ & UPR2 ESTUARY & 35 & 48 & $06 / 12-07 / 04 / 82$ \\
\hline
\end{tabular}


Table 8.--(Coatinued)

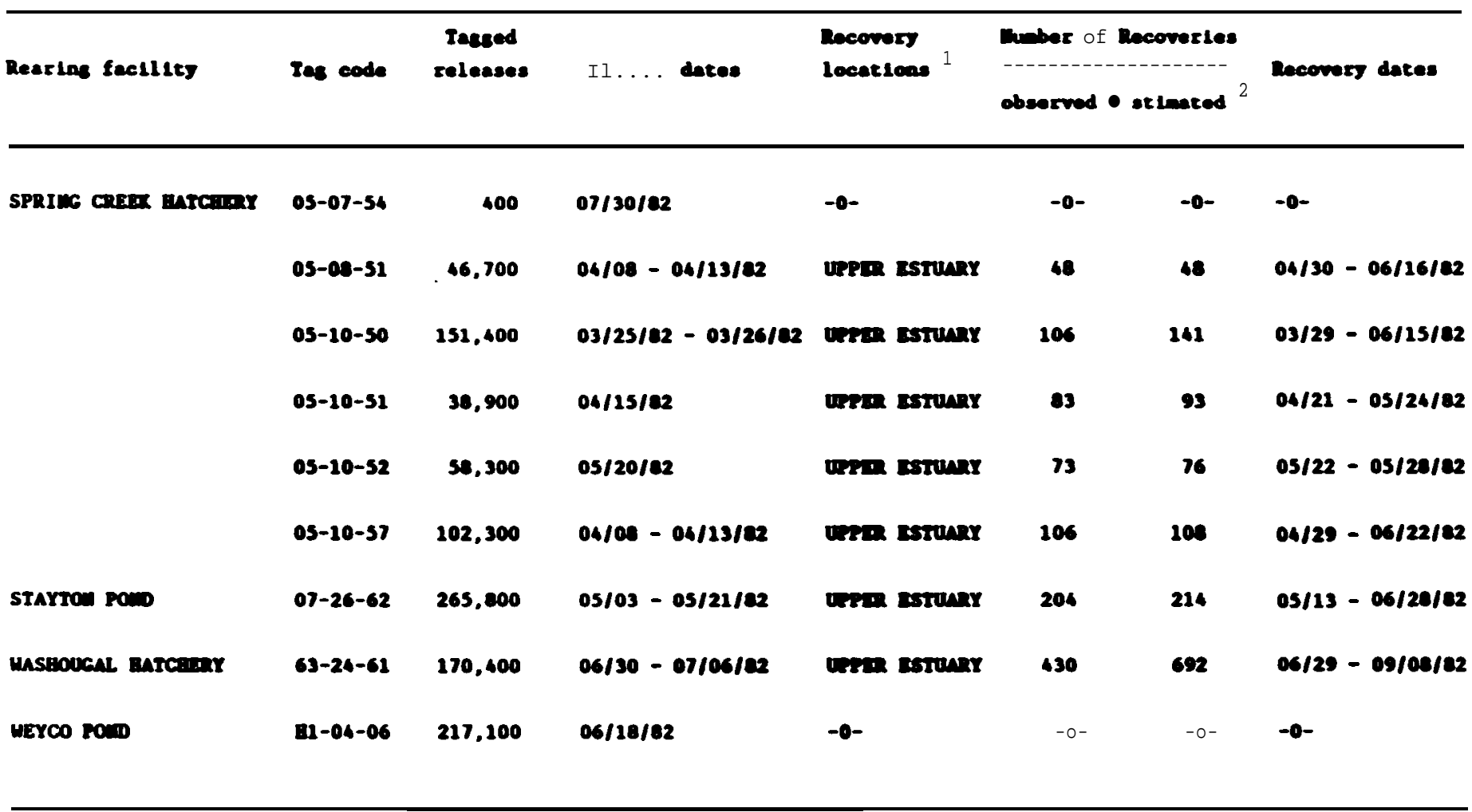

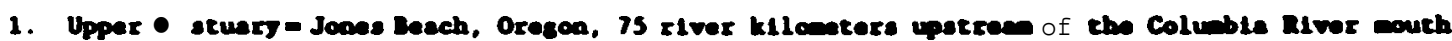
Lover estuary = 14 to 43 kllometers upstreen of the Columble River nouth

Ocene = earine areas edjecent to the Colubble River mouth

2. Standardized for fishlns effort at Jones Banch 
Figure 7.-- Proportion of fall chinook salmon from Columbia River rearing facilities caught in Pacific coast fisheries by area of catch and brood year.
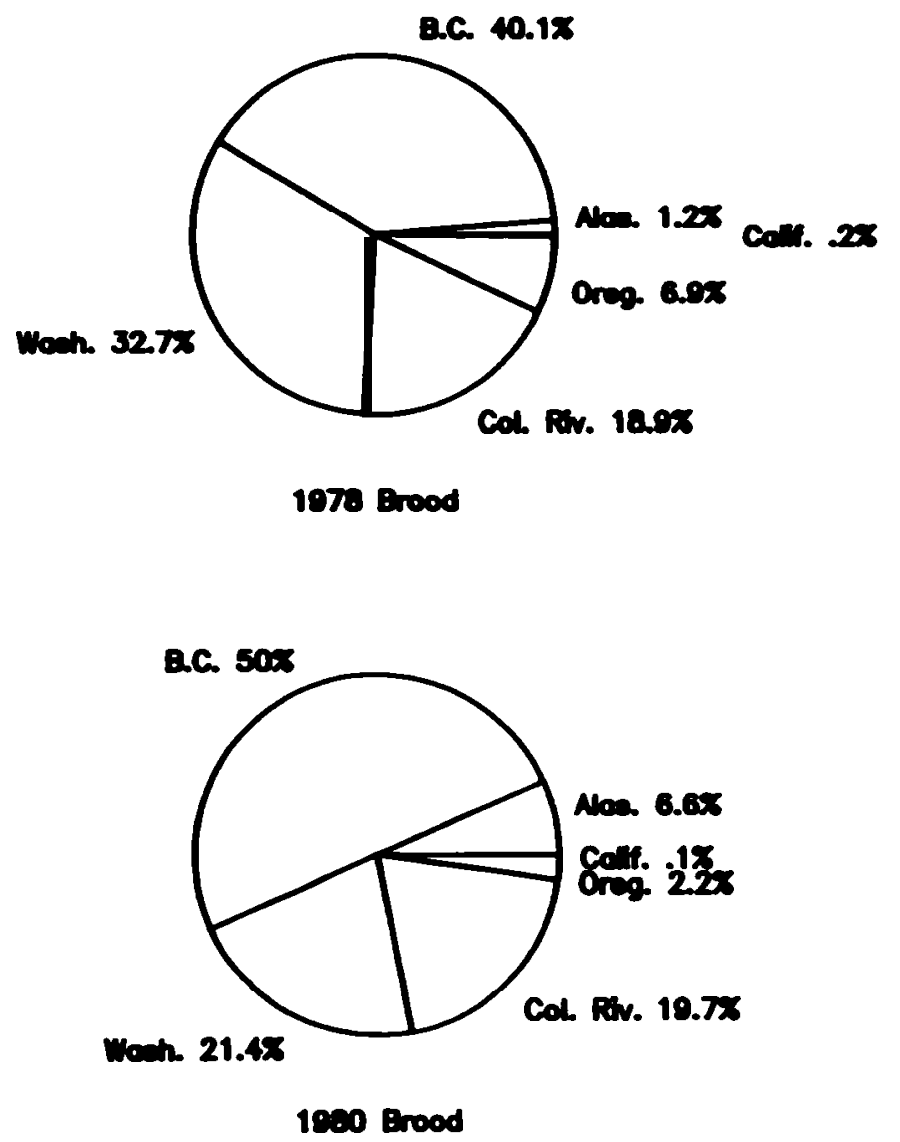

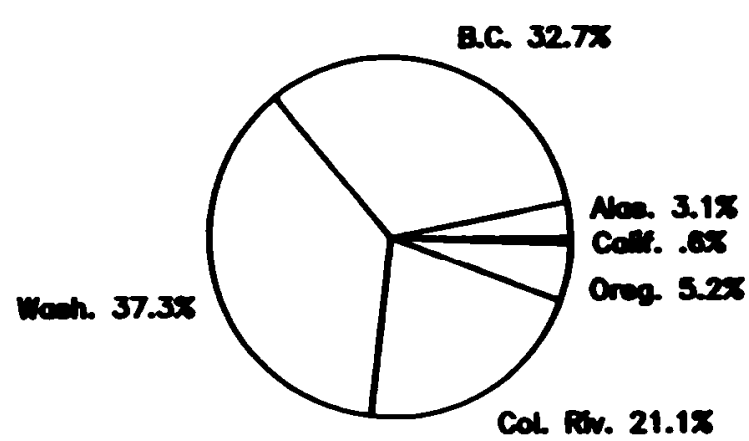

1979 Brood

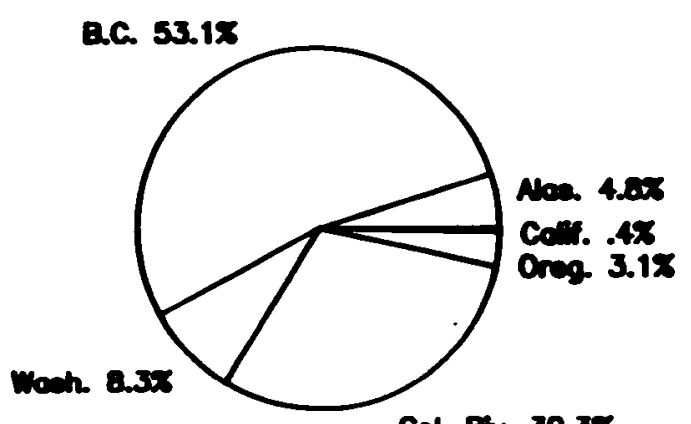

Col. Por. 30.35

1901 Brood 
Alask Elaher1es proportion range froma low of $\mathbf{1 . 2 8}$ for the 1978 brood to a high of $\mathbf{6 . 6 8}$ for the $1980 \mathrm{brood}$. The proportion8 in Brit1sh Columbla range from a low of 32.7 for the 1979 brood to a high of $53.1 *$ forthe 1981 brood. The range for Washington fleherles was 8.3: for the $1981 \mathrm{brood}$ to 37.3 for the $1979 \mathrm{brood}$. The range for the Oregon f1sherles was 2.28 for the 1980 brood to 6.9 for the $1978 \mathrm{brood}$. In the California flsheries the proportions rang8 from 0.1 for the 1980 brood to $\mathbf{0 . 6 2}$ for the $1979 \mathrm{brood}$. Proportion ranged for the Columbla RIver fisheries - 818.9 for the 1978 brood to 30.3 for the 1981 brood. There - ppears to be a trend toward higher contribution 8 to the nore northerly flsherles for the last two brood years of fall chinook salmon tagsed, 1980 and 1981. The proportion 8 of the contribution by brood and hetchery $\bullet$ ra presented in Append1x Tables $1-4$.

The proportion8 Of contribution to the flohery arees by rearing fac1l1ty follow trends similar to the brood year averages. However, trende for individual hatcheries may be influenced by low numbers of recoveries. The Canadian recovery proportion decreased from the 1978 brood to the 1979 brood and then Increased again for the 1980 and 1981 broods.

klickltat and Priest Rapide hatchery do not follow this general $\bullet$ rend in that the Canadian proportion of the recoverles increased for the 1980 brood and then decreased for the $\mathbf{1 9 8 1}$ brood. The Canadian proportion of the recover10e Increase stead1ly from the 1978 through the 1981 brood8 : for tagged flsh frombeshougal and Sea Resources hetchery. The low number of recovertes (25) for the 1978-brood fish fron see Resources Hatchery again may have Influenced this difference in trand. Bxcopt for the 1979-brood fall chinook salmon fron Sea Resources Hatehery and 1980. brood flsh fromAbermathy Hatchory, only Washington Deparenent of Fisheries hatcherles contribute to the Alaske flsheries. The Alaskan flshery recovery proportion was greatest for Priest Raplda Hatchery, ranging from 18: to $\mathbf{4 1}$ for the four brood years.

For all rearling facilities and brood years combined, the 3-year-old fall chinook salmon contributed most heavily to the sport andeomerelal flsherles (648), followed by the 4-year-old flsh (248), the 2-year-olds (88), and the 5-year-01ds (38). Lo88 than 0.18 of the floh were recovered in the flsherles as 6 -year-olds. The rang 8 over the four brood yearsare: 6.1 to 11.98 for the 2-year-01ds, 54.7 to 69.7 for the 3-year-olds, 17.9 to 32.1 for the 4-year-olds, 1.1 to 5.5 for the 5 year-olds, and 0.1 to 0.2 for the 6 -year-olds (FIgure 8). There 0 ppear to be differences anong groupe Of facllities when grouped by operating agency (F1gure 9). For USFWS and ODFW facllitles, the 3-year-old fall chinook salmon contributed more heavily than the - verage,78 and 778 respectively. At USFWS facilitles, the 3-year-old contribution is followed bythe 2-yeer old8 (128), the 4-year-olds (108), and 5-yearold8 (0.28). At ODFW facilities, the 3-year-old contribution 1s followed by 4-year-olds (158), 2-year-olds (8s), and 5-year-olda $(0.68)$. At WDF faclilties, the contribution by 0 ge 1 quit 8 differentfom the other two agencies. The 4-year-old flsh contribute the most heav1ly (528), followed by the 3-year-olds (35t), 5-year-olds (9s), 2-year-olds (48), and 6-year-olds $(0.26)$. Individual factlities generally follow the same - pparent trend as the agency group8 (Appendix Tables 5-8).

The age distributions of the flshery recover1es 0 ppear to vary with the brood. For all faclilties combined, there is a predominance of 3-year- 


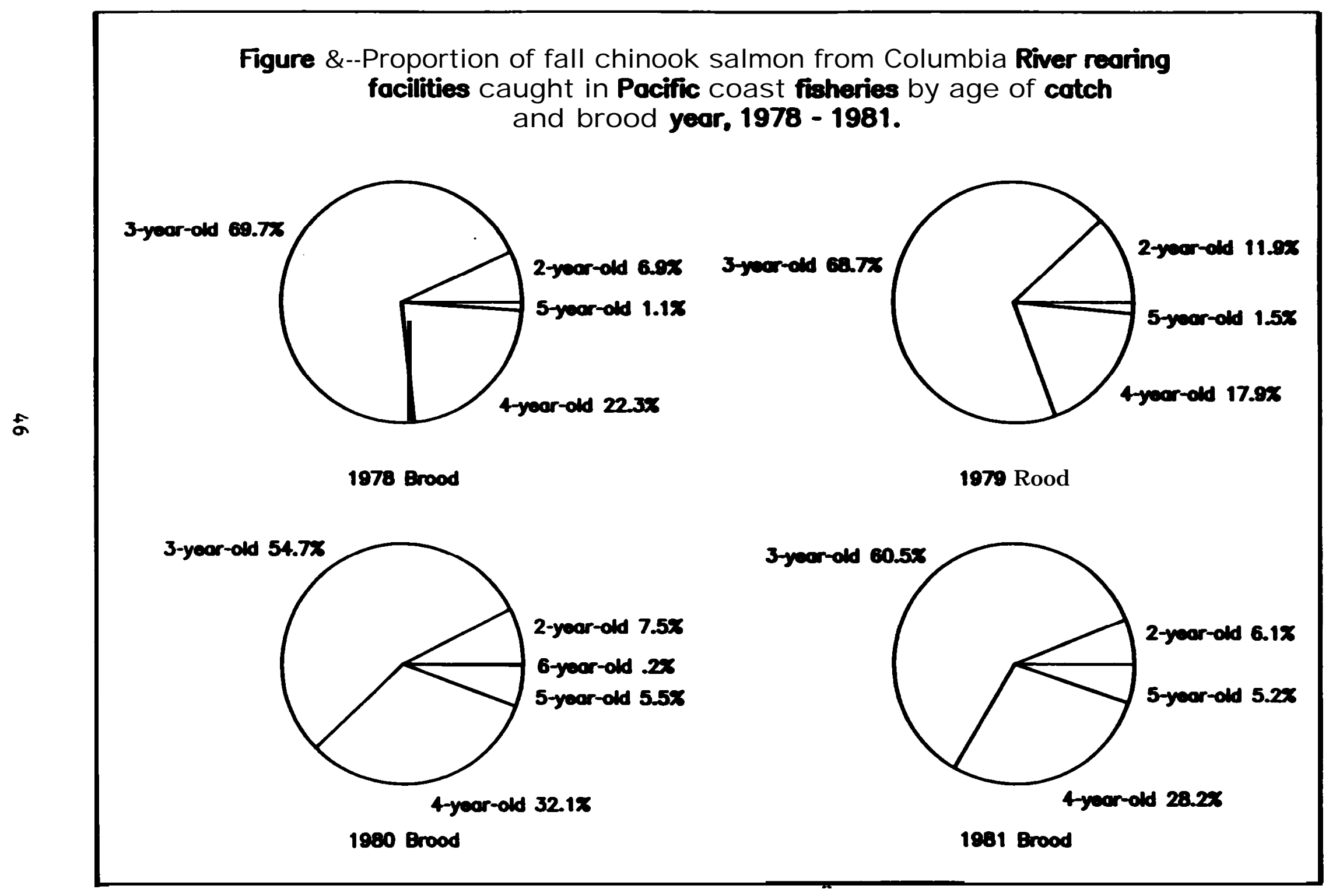


Figure 9.--Proportion of fall chinook solmon from Columbia River rearing facilities caught in Pacific coast fishereies by oge of catch for all brood years and focilities combined and by operating agency.

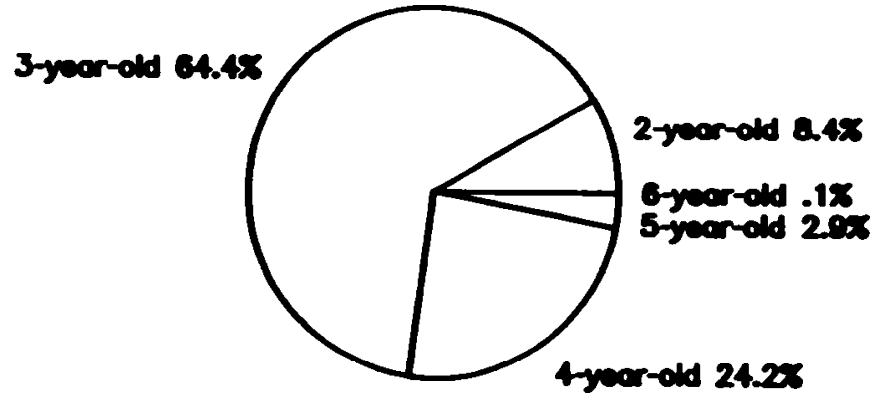

Conthed

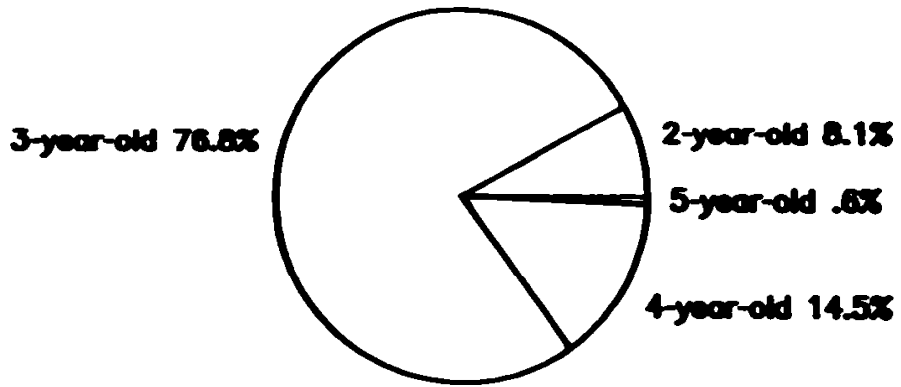

OOFI.

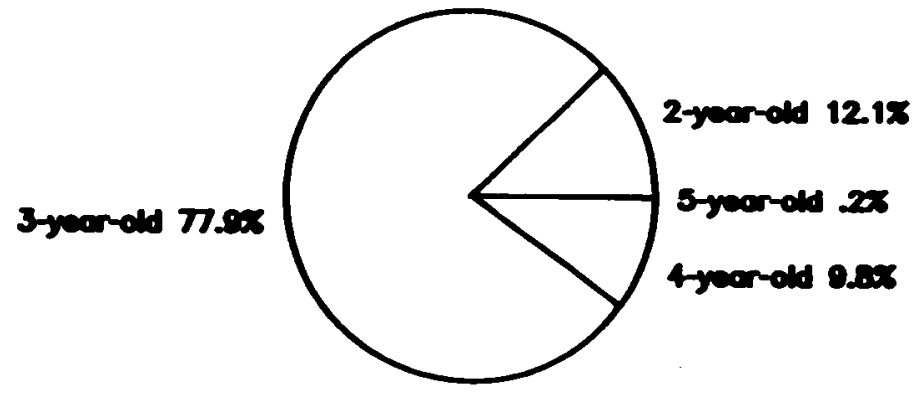

usro

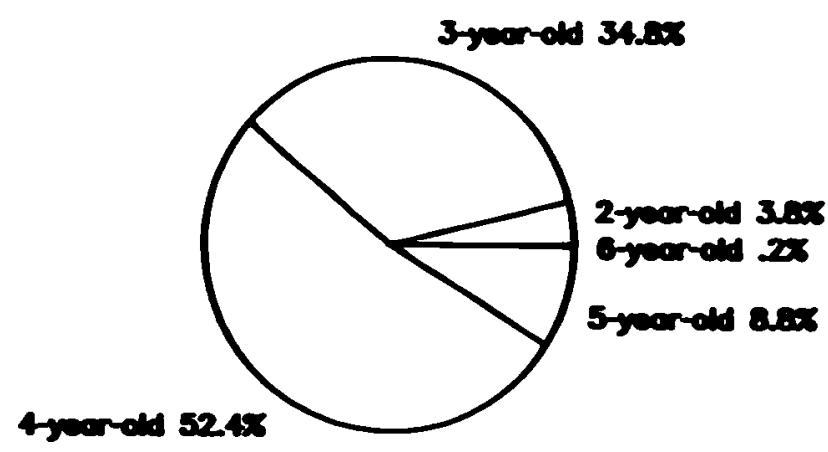

ex 
old flsh in the recoverles of the 1978 and 1979 broods (nearly 70 of the catch). The 4-year-old catch 1a about 208 for the ane two broods. For the 1980 and 1981 broods, the 3-year-old recoveries decreased to 55 and 61 reapectively, whlle the 4-year-old recoverles Increased to 32 and 28 respectively ( $F 1$ gure 8). Thlotrendis $\square \bullet \bullet$ pparent when exanining - ge proportlon forindividual facilities. For some fac1l1t1es the 1980 or 1981 brood flshery recoverien have the greatest proportions of 4- and 5-year-olds. For other faclilties this is not trua.

F1sheryContribution

The estinated recoverles of tasged fall chinook salmon for each brood year are presented in Tables 9 through 12 by rearling facility $\sigma$ an $\Omega$ rea of recovery. The contribution of each facility to the Pacific coast oport and commerclal flaher les 1s estimated fron the total eatimated fluhery recover1es of tagged floh fron each rearing facllity using the mothod described in the METHODS AND MTERIALs section (Tab188 13 - 16). It 1sestimated that $\mathbf{1 , 0 2 0 , 8 0 0 ~ f a l l ~ c h i n o o k ~ s a l n o n ~ f r o m ~ t h e ~ C o l u m b i a ~}$ rearing faclilties wore caught in the marine and freshwater sport and comercial floheries during this study. This la an average of 2.9 flsh caught for every 1000 fleh released over the four brood years.

The flahery contribution per 1000 flsh releaned ranged from a low of 1.9 for the $1980 \mathrm{brood}$ to a high of 4.7 for the $1979 \mathrm{brood}$ ( 1 1gure 10). The contribution8 - ppear to be slightly differentamong broods and quit 8 different anong fac1lieles within a brood (F1gures $\mathbf{1 1}$ through $\mathbf{1 4}$ and Appendix Tables 13 through 16). Spring Creek Hetchery had the greatest E1shery contribution for the 1978 and 1979 brood8 (8.6 and 12.7 f1sh per 1000 flsh released). For the 1980 and 1981 broods, Abernathy and Sea Resources hatcherles had the greatest contribution per 1000 f1sh released ( 7.8 and 6.0 respoctively). The contribution for Spring Creek Hetchery dropped to 1.9 and 3.1 per 1000 flsh released for the 1980 and 1981 brood8 respectively (FIgures 13 and 14). The contribution for Priest Rapid8 Hatchery Increased steadlly over the four broods, going from a low of 2.3 per 1000 f1sh releesed for the 1978 brood to a high of 4.7 for the 1981 brood. The flshery contribution8 for Cowl1tz, Elokomin, and Lower Kalama hatcheries were greatest for the 1980 and 1981 broods.

Spring Creak Hatchery contributed a large proportion (55 and 52*) of the total flshery contribution fromell chinook selmon facilities on the Columbia River for the 1978 and 1979 brood8 respectively (F1gures 15 and 16). Releases from Spring Creek Hatchery were 21 and 19 o fhe total releases for the two brood years respectively. The noxt greatest proportion8 were 128 from Bonneville Hatchery (1978 brood) and 148 from Big Creek Hatchery (1979 brood). For the 1978 brood, $\mathbf{7 . 5}$ of the fall chinook salmon releases from Columbia River facilities came from Bonnev11le Hatchery. For the 1979 brood, 83 of the release8 came from Big Creek Hatchery. The Spring Creek tagged fish make up $\mathbf{1 8 . 5}$ and 168 of the tot81 flsh released for the 1980 and 1981 broods respectively (Tables 3 and 4). The fishery contribution proportions of these same Elsh are 19 and 25: for the same broods respectively (Figures 17 and 


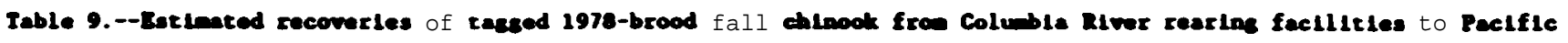
const fisheries by facllity.

\begin{tabular}{|c|c|c|c|c|c|c|c|c|c|c|}
\hline \multirow{3}{*}{ Rearing facllity } & \multicolumn{10}{|c|}{ Hubers of Recoverles } \\
\hline & \multicolumn{6}{|c|}{ Marten } & \multicolumn{2}{|c|}{ Columbla River } & \multicolumn{2}{|c|}{ Total } \\
\hline & Alacka & Canada & Washingtea & Oreagen & Callfornla & Forelen & Indian & Won-Indlan & $\mathbf{A 1 1}$ & - flah. \\
\hline Anentux ancent & 0 & 276 & 140 & 45 & $\mathbf{s}$ & 0 & 0 & $\boldsymbol{\omega}$ & & 532 \\
\hline BIG cater entcian & 0 & 364 & 182 & 27 & 0 & 0 & 0 & $\mathbf{8 6}$ & & 659 \\
\hline DIG UATE POD & 0 & 79 & 133 & 21 & 0 & 0 & 29 & 12 & & 274 \\
\hline 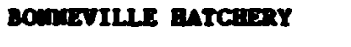 & 0 & 393 & 292 & 57 & 4 & 0 & 15 & 93 & & ess \\
\hline Coinlit2 batcianY & 11 & 105 & 115 & 46 & 0 & 0 & 0 & 32 & & 309 \\
\hline Elozoury Butchixay & 0 & 10 & 3 & 0 & 0 & 0 & 0 & 4 & & 17 \\
\hline CraYs RIVER BatchidnY & 9 & 8 & 17 & 13 & 0 & 0 & 0 & 24 & & 71 \\
\hline ruLAMA PALLS BATCBIXRY & 4 & 47 & 16 & 3 & 0 & 0 & 0 & 23 & & 91 \\
\hline 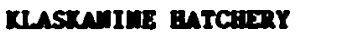 & 0 & 140 & 33 & 34 & 0 & 0 & 0 & 105 & & 312 \\
\hline ILICXITAT BATCaIRY & 2 & 106 & 72 & 7 & 0 & 0 & 31 & 20 & & 243 \\
\hline LITTLE MITE BATCaERY & 0 & 23 & 22 & 5 & 0 & 0 & 16 & 0 & & 66 \\
\hline PRIEST RAPIDS BATCuEXY & 73 & 82 & 5 & 3 & 4 & 0 & 16 & $\bullet$ & & 191 \\
\hline SEA RESOURCES BATcalary & 0 & 5 & 3 & 12 & 0 & 0 & 0 & 5 & & 25 \\
\hline
\end{tabular}


Table 9.--(Coat lound)

\begin{tabular}{|c|c|c|c|c|c|c|c|c|c|}
\hline \multirow{3}{*}{ Rearias facllity } & \multicolumn{9}{|c|}{ Mubbers of Recoverles } \\
\hline & \multicolumn{6}{|c|}{ Marlan } & \multicolumn{2}{|c|}{ Columble River } & \multirow{2}{*}{$\begin{array}{c}\text { Total. } \\
\text { Al flah. }\end{array}$} \\
\hline & Alaska & Caneds & Uashinatea & Oregen & Callfornia & Forelen & Indlen & Von-Indlian & \\
\hline SPEELYAI BATCRARY & 25 & 110 & 102 & 20 & 0 & 0 & 0 & 6 & 325 \\
\hline SPRIMG CREEX BATCEIERY & 0 & 1621 & 1868 & 340 & 0 & 2 & 914 & 299 & 5044 \\
\hline staytow Pow & 0 & 974 & 631 & 121 & 7 & 2 & 0 & 249 & 1884 \\
\hline TOUTLE BATCaERY & 5 & 76 & 12 & 15 & 0 & 0 & 0 & 29 & 135 \\
\hline WASHCUGAL BATCHERY & 8 & 111 & 55 & 18 & 0 & 0 & 0 & es & 277 \\
\hline WEYCO POWD & 2 & 37 & 10 & 0 & 0 & 0 & 0 & 26 & 65 \\
\hline Total & 139 & 4565 & 3714 & 787 & 20 & 4 & 1021 & 1124 & 11,374 \\
\hline
\end{tabular}




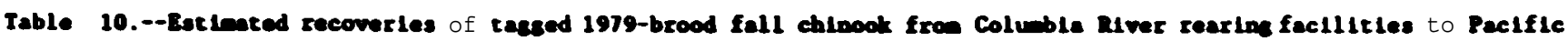
coast flaburles by facllity.

\begin{tabular}{|c|c|c|c|c|c|c|c|c|c|}
\hline \multirow{3}{*}{ Rearins fecllity } & \multicolumn{9}{|c|}{ Hubers of Recoveries } \\
\hline & \multicolumn{6}{|c|}{ Marine } & \multicolumn{2}{|c|}{ Columble River } & Total \\
\hline & Alask & Canada & Washingten & Oregen & Callfornle & Forelen & Indian & Mon-Indlan & All fish. \\
\hline 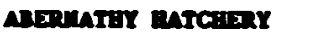 & 0 & 201 & 348 & $\boldsymbol{c s}$ & $\mathbf{a}$ & 5 & 0 & es & 723 \\
\hline big carex matciany & 0 & 595 & 384 & s9 & $\mathbf{a}$ & 4 & 0 & 160 & 1210 \\
\hline 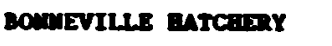 & 0 & 75 & 30 & 0 & 0 & 0 & 2 & 24 & 139 \\
\hline COALIT2 Butcisir & 28 & 161 & 317 & 20 & 0 & 0 & 0 & 73 & 607 \\
\hline DLozary Batciser & 0 & 23 & 15 & 12 & 0 & 2 & 0 & 12 & 64 \\
\hline Gays RIVE atcusay & 7 & 37 & 12 & 2 & 0 & 0 & 0 & 7 & 65 \\
\hline raLara PALLS aurcagery & 12 & 106 & 65 & 9 & 0 & 0 & 0 & 18 & 210 \\
\hline 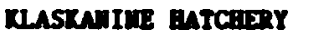 & 0 & 34 & 20 & 3 & 0 & 0 & 0 & 36 & 93 \\
\hline Xlcurat batcaixny & 10 & 97 & 83 & 14 & 17 & 0 & 19 & 19 & 249 \\
\hline LEUIS RIVER gutcasay & 21 & 168 & 108 & 37 & 0 & 0 & 0 & 121 & 455 \\
\hline LITTLE MarTE EATCBexy & 0 & 18 & 20 & 0 & 0 & 0 & 2 & 6 & 46 \\
\hline LOUER TALMA BATCHERY & 8 & 86 & 32 & - & 0 & 0 & 0 & $\mathbf{s 1}$ & 185 \\
\hline OXsow entcateny & 0 & 78 & 48 & 11 & 0 & 0 & 0 & 22 & 159 \\
\hline
\end{tabular}


Table 10.--(Coatlound)

\begin{tabular}{|c|c|c|c|c|c|c|c|c|c|}
\hline \multirow{3}{*}{ Rearlas feclltty } & \multicolumn{8}{|c|}{ Mubers of Recoverles } & \multirow[b]{2}{*}{ Total } \\
\hline & \multicolumn{4}{|c|}{ Merim } & & & \multicolumn{2}{|c|}{ Coluble Revex } & \\
\hline & Alaska & Canuda & Uashingtoo & Oresen & Califormie & Forelen & Indlea & Non-Indien & All flah. \\
\hline PRIEST RAPIDS aATCiar & 181 & 149 & 20 & 9 & 0 & 0 & 42 & 37 & 430 \\
\hline SEA RESOUCES BATCixir & 12 & 22 & 7 & 0 & 0 & 0 & 0 & 11 & 52 \\
\hline SPRINC Centir matcainy & 0 & 785 & 1431 & 179 & 23 & 2 & 723 & 503 & 3646 \\
\hline starrou POS & 0 & 641 & sos & 116 & 3 & 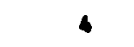 & 0 & 221 & 1890 \\
\hline unsmoven mercisery & 63 & 339 & 244 & 17 & 10 & 0 & 18 & 107 & 790 \\
\hline MEYCO POND & 1 & 20 & 45 & 2 & 0 & 0 & 0 & 26 & 96 \\
\hline Total & 343 & 3625 & 4142 & 372 & 69 & 17 & 206 & 1539 & 11,113 \\
\hline
\end{tabular}




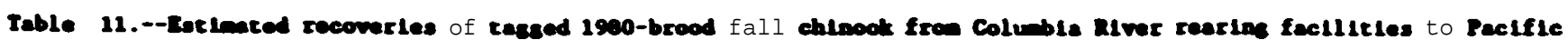
canet fleberles by facility.

\begin{tabular}{|c|c|c|c|c|c|c|c|c|c|c|}
\hline \multirow{3}{*}{ Dearlng feellity } & \multicolumn{10}{|c|}{ Mubers of Iecoveries } \\
\hline & \multicolumn{6}{|c|}{ Mrion } & \multicolumn{2}{|c|}{ Columbla River } & \multicolumn{2}{|c|}{ Total } \\
\hline & Alaske & Canoda & Weshingrea & Orepen & Colsfornle & Porelen & Indien & Woa-Indlan & $\mathbf{A 1}$ & flah. \\
\hline Anxinater entceny & 2 & 308 & 265 & $1 \mathrm{~b}$ & 0 & 0 & 4 & 48 & & 642 \\
\hline sic coner antoint & 0 & 178 & $\mathbf{e 1}$ & 9 & 0 & 0 & 0 & 36 & & 304 \\
\hline 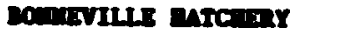 & 0 & 233 & 102 & $1 \mathrm{~b}$ & 0 & 0 & 9 & 30 & & 300 \\
\hline Cutsol court roms & 0 & 191 & $\boldsymbol{e 4}$ & 5 & 0 & 0 & 0 & 69 & & 349 \\
\hline contitz entoing & 40 & 390 & 122 & 37 & 4 & 0 & 0 & 140 & & 742 \\
\hline zocourn enta: ix & 12 & 111 & 22 & 11 & 0 & 0 & 0 & 54 & & 210 \\
\hline Gars arve arcion & 11 & 86 & 21 & 2 & 0 & 0 & 0 & 30 & & 140 \\
\hline ralay rals arcolder & 26 & 114 & 30 & 1 & 0 & 0 & 0 & 25 & & 196 \\
\hline answare ano: iny & 0 & 31 & 8 & 0 & 0 & 0 & 0 & 29 & & $\omega$ \\
\hline dratrat miciax & 2 & 20 & 1 & 1 & 0 & 0 & 11 & 0 & & 43 \\
\hline LITIS wIT marcisnx & 8 & 26 & 17 & $\boldsymbol{0}$ & 0 & 0 & 10 & 5 & & 36 \\
\hline Lover rume mitcidar & 76 & 330 & 53 & 10 & 0 & 0 & 0 & 150 & & 627 \\
\hline 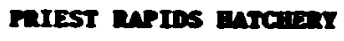 & 294 & 491 & 15 & 9 & 0 & 2 & 156 & 56 & & 1021 \\
\hline
\end{tabular}


Table 11.--(Contlomed)

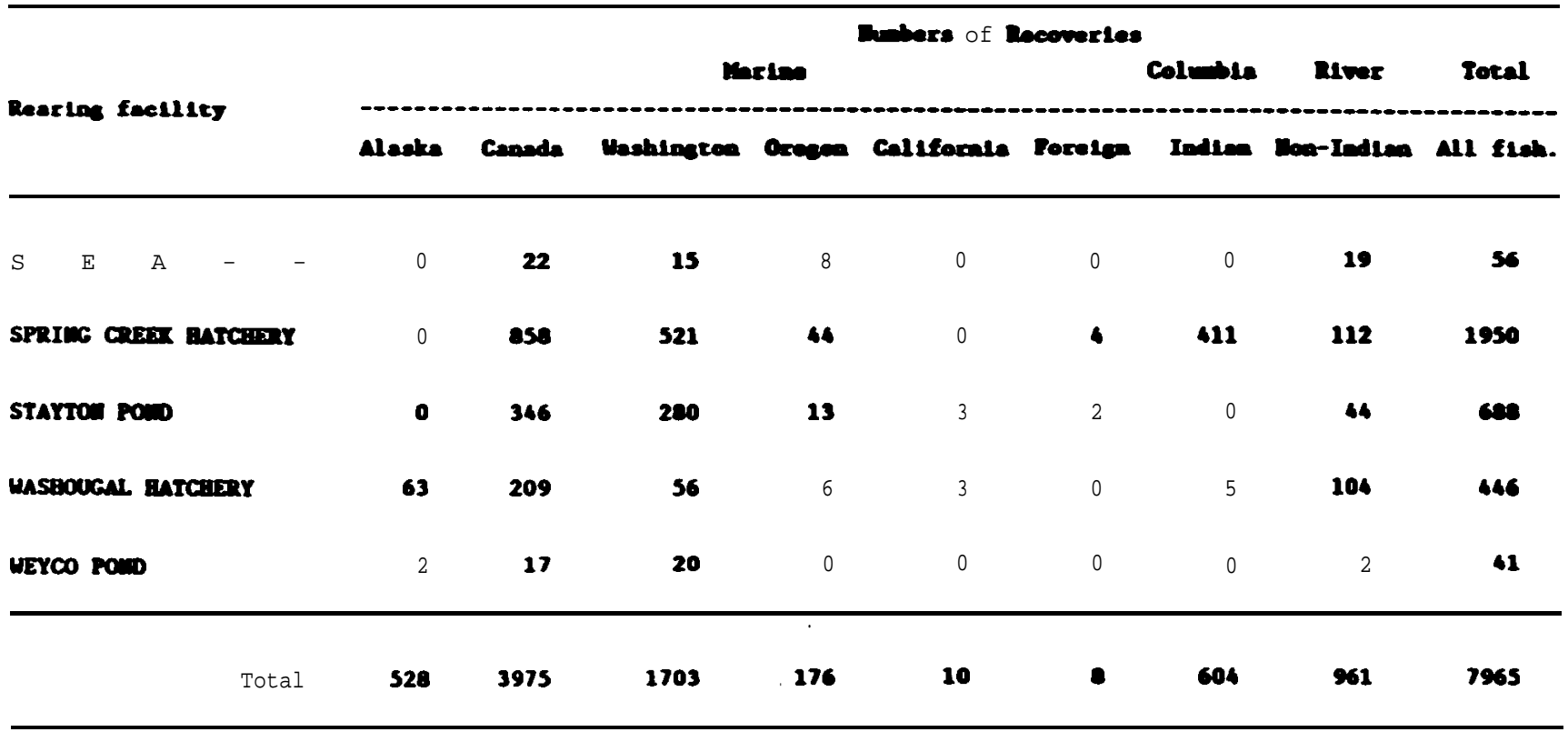


Table 12.--Retianted recoverles of taged 19a1-brood fall chinock fron Columbla River rearing facllities to Paclfle coant flsherles by factllty.

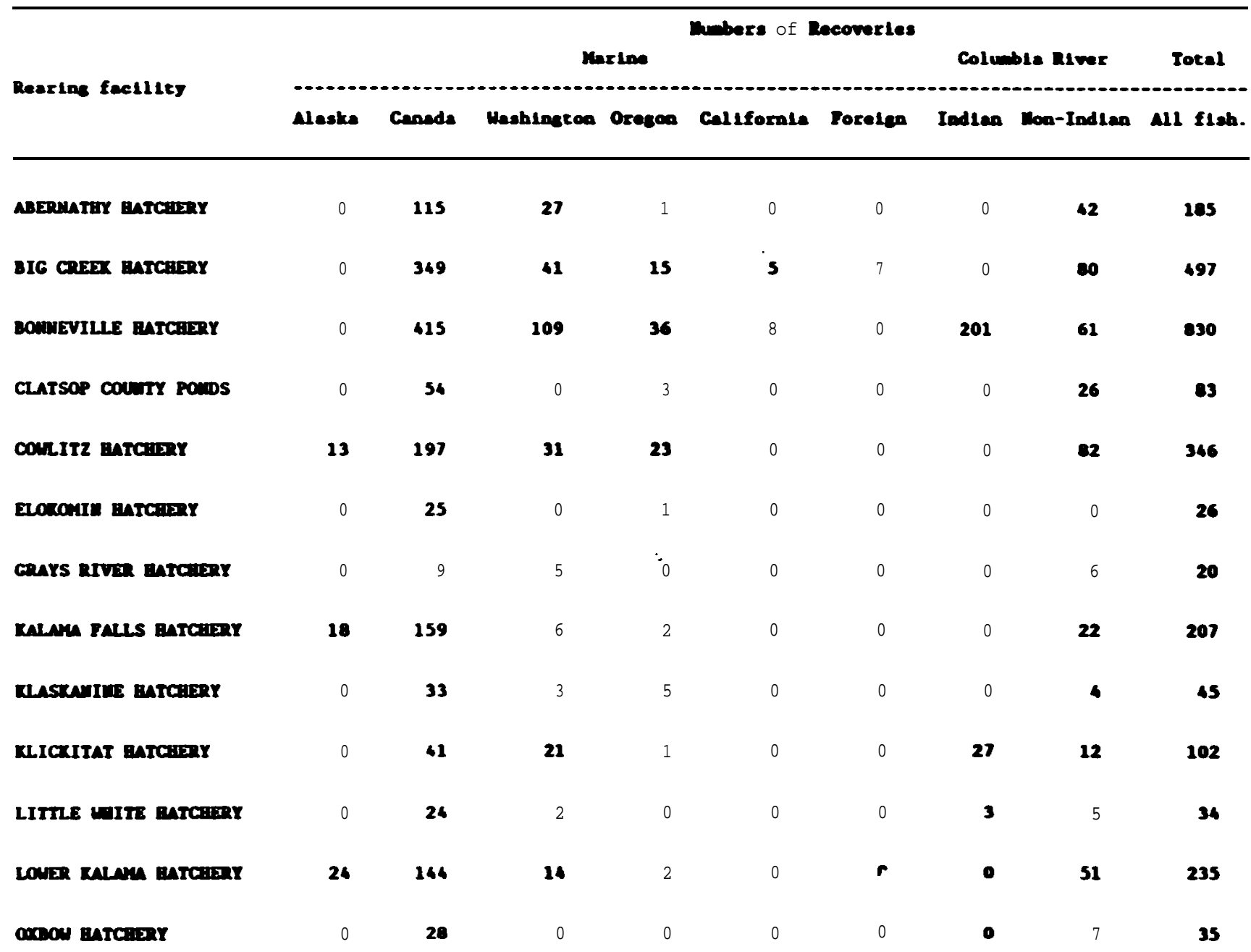


Table 12.--(Cone1mad)

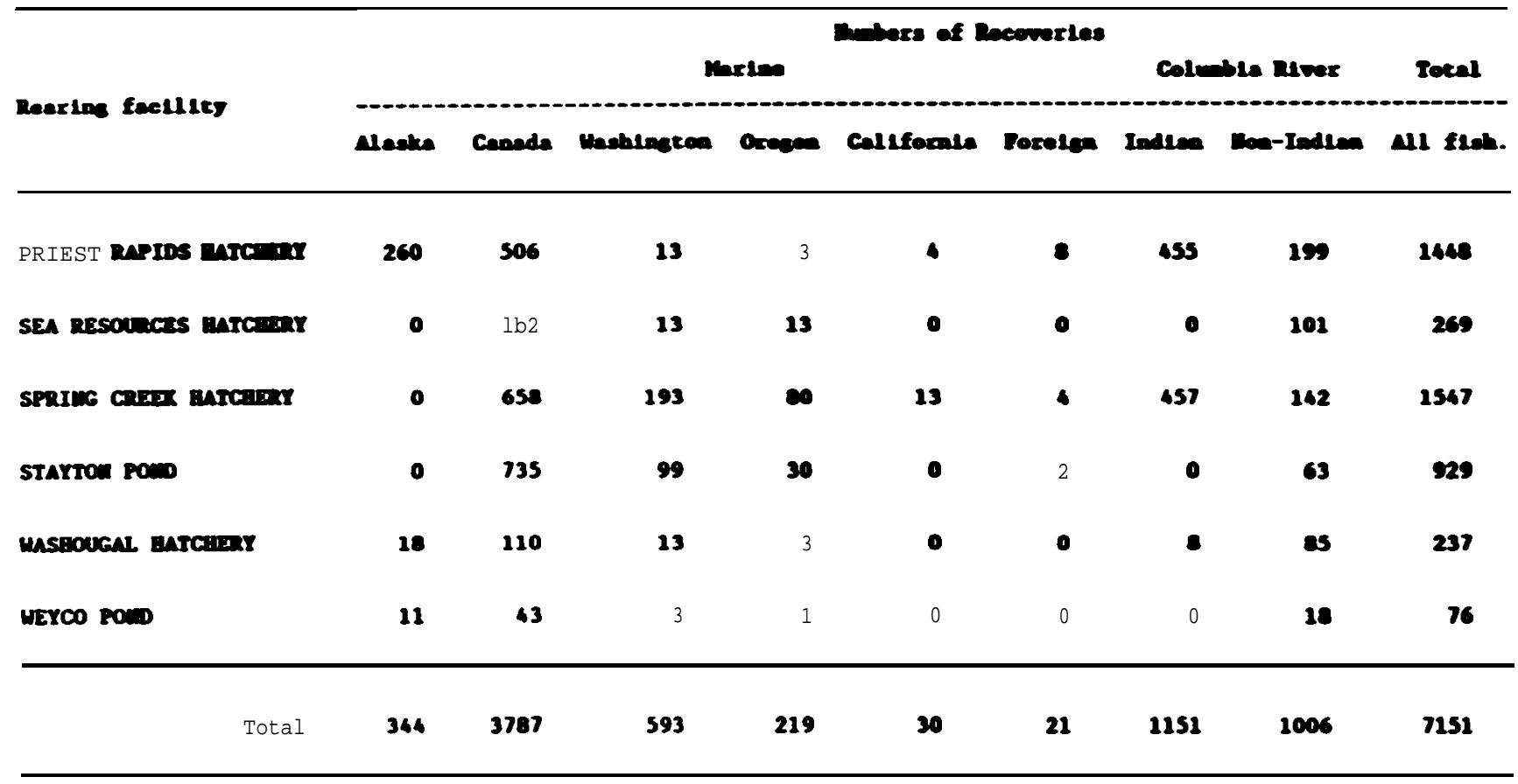




\begin{tabular}{|c|c|c|c|}
\hline Rearing facility & $\begin{array}{l}\text { Tagged } \\
\text { catch }\end{array}$ & $\begin{array}{c}\text { Total } \\
\text { contribution }\end{array}$ & $\begin{array}{l}\text { Contribution } \\
\text { per } 1000 \text { releases }\end{array}$ \\
\hline ABERNATHY HATCHERY & 532 & 7.635 & 4.74 \\
\hline BIG CREEK HATCHERY & 659 & 15,378 & 2.93 \\
\hline BIG WHITE POND & 274 & 5,872 & 1.94 \\
\hline BONNEVILLE HATCHERY & 854 & 37,386 & 2.79 \\
\hline COWLITZ HATCHERY & 309 & 8,033 & 1.73 \\
\hline ELOKOMIN HATCHERY & 17 & 273 & 0.09 \\
\hline GRAYS RIVER HATCHERY & 71 & 452 & 0.33 \\
\hline KALAMA FALIS HATCHERY & 91 & 1,782 & 0.43 \\
\hline KLASKANINE HATCHERY & 312 & 7,019 & 1.28 \\
\hline KLICKITAT HATCHERY & 243 & 3,880 & 1.08 \\
\hline LITTLE WHITE HATCHERY & 66 & 1,745 & 0.15 \\
\hline PRIEST RAPIDS HATCHERY & 191 & 2,794 & 2.33 \\
\hline SEA RESOURCES HATCHERY & 25 & 1,014 & 1.03 \\
\hline SPEELYAI HATCHERY & 325 & 464 & 1.95 \\
\hline SPRING CREEK HATCHERY & 5,044 & 167,237 & 8.55 \\
\hline STAYTON POND & 1,884 & 31,179 & 6.65 \\
\hline TOUTLE HATCHERY & 135 & 2,675 & 0.97 \\
\hline WASHOUGAL HATCHERY & 277 & 6,801 & 1.34 \\
\hline WEYCO POND & 65 & 258 & 0.70 \\
\hline All facilities & 11,374 & 301,878 & 3.29 \\
\hline
\end{tabular}


Table 14.-- Tagged catch and contribution of 1979-brood fall chinook salmon to all Pacific coast fisheries by rearing facility.

\begin{tabular}{|c|c|c|c|}
\hline Rearing facility & $\begin{array}{r}\text { Tagged } \\
\text { catch }\end{array}$ & $\begin{array}{c}\text { Total } \\
\text { contribution }\end{array}$ & $\begin{array}{l}\text { Contribution } \\
\text { per } 1000 \text { releases }\end{array}$ \\
\hline ABERNATHY HATCHERY & 713 & 9,575 & 4.84 \\
\hline BIG CREEK HATCHERY & 1,210 & 54,289 & 8.44 \\
\hline BONNEVILLE HATCHERY & 139 & 5.826 & 1.15 \\
\hline COWLITŻ HATCHERY & 607 & 15,470 & 1.90 \\
\hline ELOKOMIN HATCHERY & 64 & 1,569 & 0.65 \\
\hline GRAYS RIVER HATCHERY & 65 & 1,399 & 1.73 \\
\hline RALAMA FALIS HATCHERY & 210 & 5,031 & 2.09 \\
\hline KLASKANINE HATCHERY & 93 & 3,139 & 1.40 \\
\hline KLICKITAT HATCHERY & 249 & 5.010 & 1.60 \\
\hline LEWIS RIVER HATCHERY & 455 & 1,877 & 4.40 \\
\hline LITTLE WHITE HATCHERY & 46 & 2,485 & 0.28 \\
\hline LOWER KALAMA HATCHERY & 185 & 4.205 & 1.28 \\
\hline OXBOW HATCHERY & 159 & 3,750 & 1.57 \\
\hline PRIEST RAPIDS HATCHERY & 438 & 9,485 & $3.98^{\circ}$ \\
\hline SEA RESOURCES HATCHERY & 52 & 2,160 & 2.83 \\
\hline SPRING CREEK HATCHERY & 3,646 & 197,680 & 12.71 \\
\hline STAYTON POND & 1,890 & 42,561 & 6.70 \\
\hline WASHOUGAL HATCHERY & 798 & 15,487 & 2.54 \\
\hline WEYCO POND & 94 & 1,876 & 0.96 \\
\hline All facilities & 11,113 & 382,873 & 4.71 \\
\hline
\end{tabular}


Table 15.- - Tagged catch and contribution of 1980-brood fall chinook salmon to all Pacific coast fisheries by rearing facility.

\begin{tabular}{|c|c|c|c|}
\hline Rearing facility & $\begin{array}{r}\text { Tagged } \\
\text { catch }\end{array}$ & $\begin{array}{c}\text { Total } \\
\text { contribution }\end{array}$ & $\begin{array}{l}\text { Contribution } \\
\text { per } 1000 \text { releases }\end{array}$ \\
\hline ABERNATHY HATCHERY & 641 & 9,359 & 7.79 \\
\hline BIG CREEK HATCHERY & 304 & 11,547 & 2.06 \\
\hline BONNEVILLE HATCHERY & 388 & 15,753 & 1.89 \\
\hline CLATSOP COUNTY PONDS & 349 & 9,462 & 3.00 \\
\hline COWLITZ HATCHERY & 741 & 16,392 & 2.65 \\
\hline ELOKOMIN HATCHERY & 210 & 2,807 & 0.96 \\
\hline GRAYS RIVER HATCHERY & 140 & 2,125 & 1.74 \\
\hline KALAMA FALLS HATCHERY & 196 & 4,039 & 1.12 \\
\hline KLASKANINE HATCHERY & 68 & 2.655 & 0.67 \\
\hline KLICKITAT HATCHERY & 43 & 820 & 0.33 \\
\hline LITTLE WHITE HATCHERY & 56 & 2,072 & 0.22 \\
\hline LOWER KALAMA HATCHERY & 627 & 12,120 & 4.04 \\
\hline PRIEST RAPIDS HATCHERY & 1,021 & 20,688 & 4.52 \\
\hline SEA RESOURCES HATCHERY & 56 & 1,075 & 1.29 \\
\hline SPRING CREEK HATCHERY & 1,950 & 31,556 & 1.90 \\
\hline STAYTON POND & 688 & 16,553 & 2.80 \\
\hline WASHOUGAL HATCHERY & 446 & 8.666 & 1.44 \\
\hline WEYCO POND & 41 & 816 & 0.17 \\
\hline All facilities & 7.965 & 168,509 & 1.88 \\
\hline
\end{tabular}




\begin{tabular}{|c|c|c|c|}
\hline Rearing facility & $\begin{array}{r}\text { Tagged } \\
\text { catch }\end{array}$ & $\begin{array}{c}\text { Total } \\
\text { contribution }\end{array}$ & $\begin{array}{l}\text { Contribution } \\
\text { per } 1000 \text { releases }\end{array}$ \\
\hline ABERNATHY HATCHERY & 185 & 2,240 & 1.54 \\
\hline BIG CREEK HATCHERY & 497 & 17,184 & 3.79 \\
\hline BONNEVILIE HATCHERY & 830 & 17,818 & 2.87 \\
\hline CLATSOP COUNTY PONDS & 83 & 2,002 & 0.73 \\
\hline COWLITZ HATCHERY & 346 & 9,875 & 1.24 \\
\hline ELOKOMIN HATCHERY & 26 & 657 & 0.25 \\
\hline GRAYS RIVER HATCHERY & 20 & 227 & 0.27 \\
\hline RALAMA FALLS HATCHERY & 207 & 4,157 & 1.17 \\
\hline KLASKANINE HATCHERY & 45 & 910 & 0.45 \\
\hline KLICKITAT HATCHERY & 102 & 1,841 & 0.50 \\
\hline LITTLE WHITE HATCHERY & 34 & 1,359 & 0.17 \\
\hline LOWER KALAYA HATCHERY & 235 & 5,348 & 1.69 \\
\hline OXBOW HATCHERY & 35 & 1,431 & 0.33 \\
\hline PRIEST RAPIDS HATCHERY & 1,448 & 25,739 & 4.69 \\
\hline SEA RESOURCES HATCHERY & 269 & 4,965 & 5.98 \\
\hline SPRING CREEK HATCHERY & 1,547 & 41,741 & 3.11 \\
\hline STAYTON POND & 929 & 23,612 & 3.50 \\
\hline WASHOUGAL HATCHERY & 237 & 4,874 & 1.39 \\
\hline HEYCO POND & 76 & 1.574 & 0.35 \\
\hline All facilities & 7.151 & 167,552 & 1.96 \\
\hline
\end{tabular}




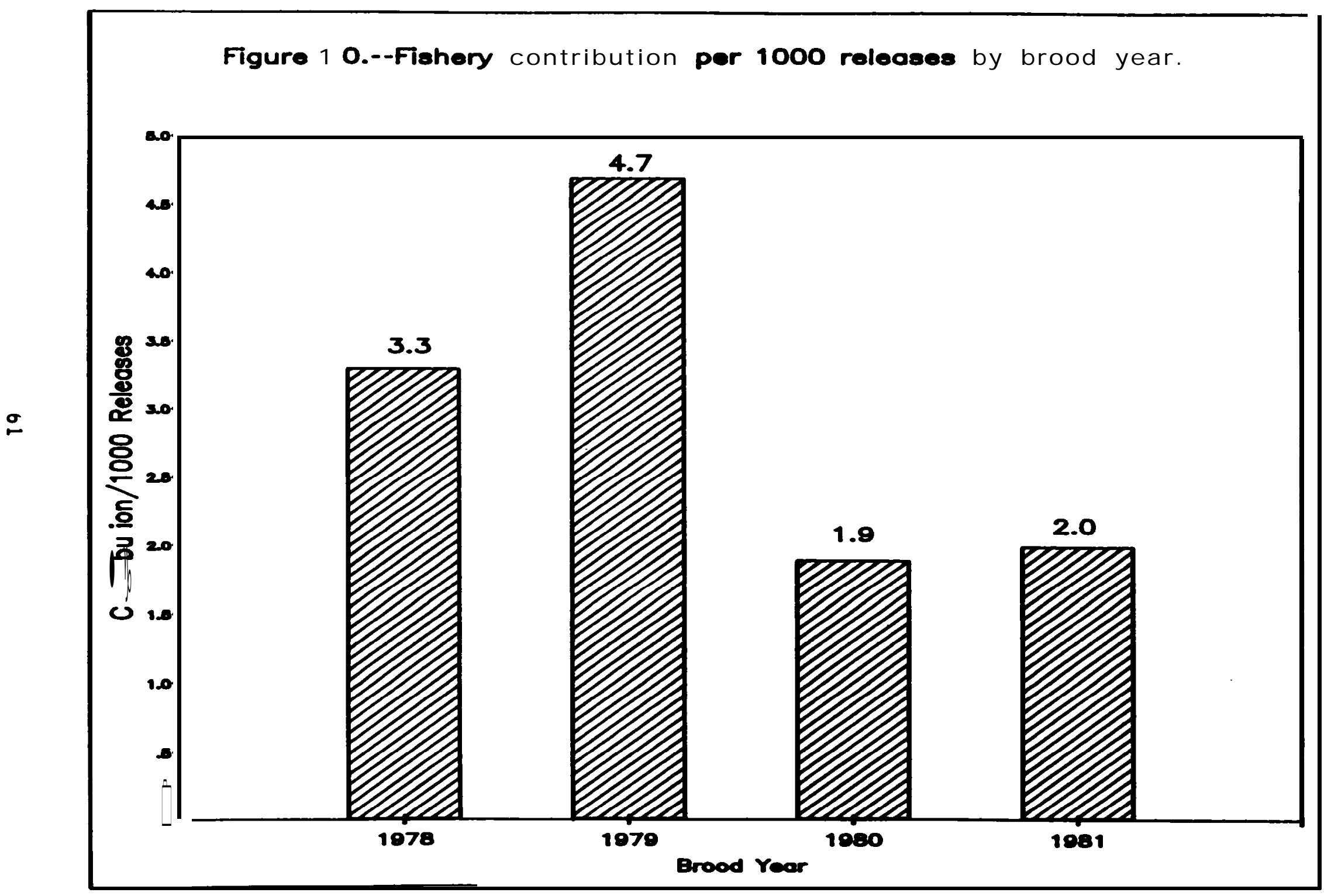


Figure 11.--Fishery contribution of the 1978-brood fall chinook aalmon from Columbia River rearing facilities by facility.

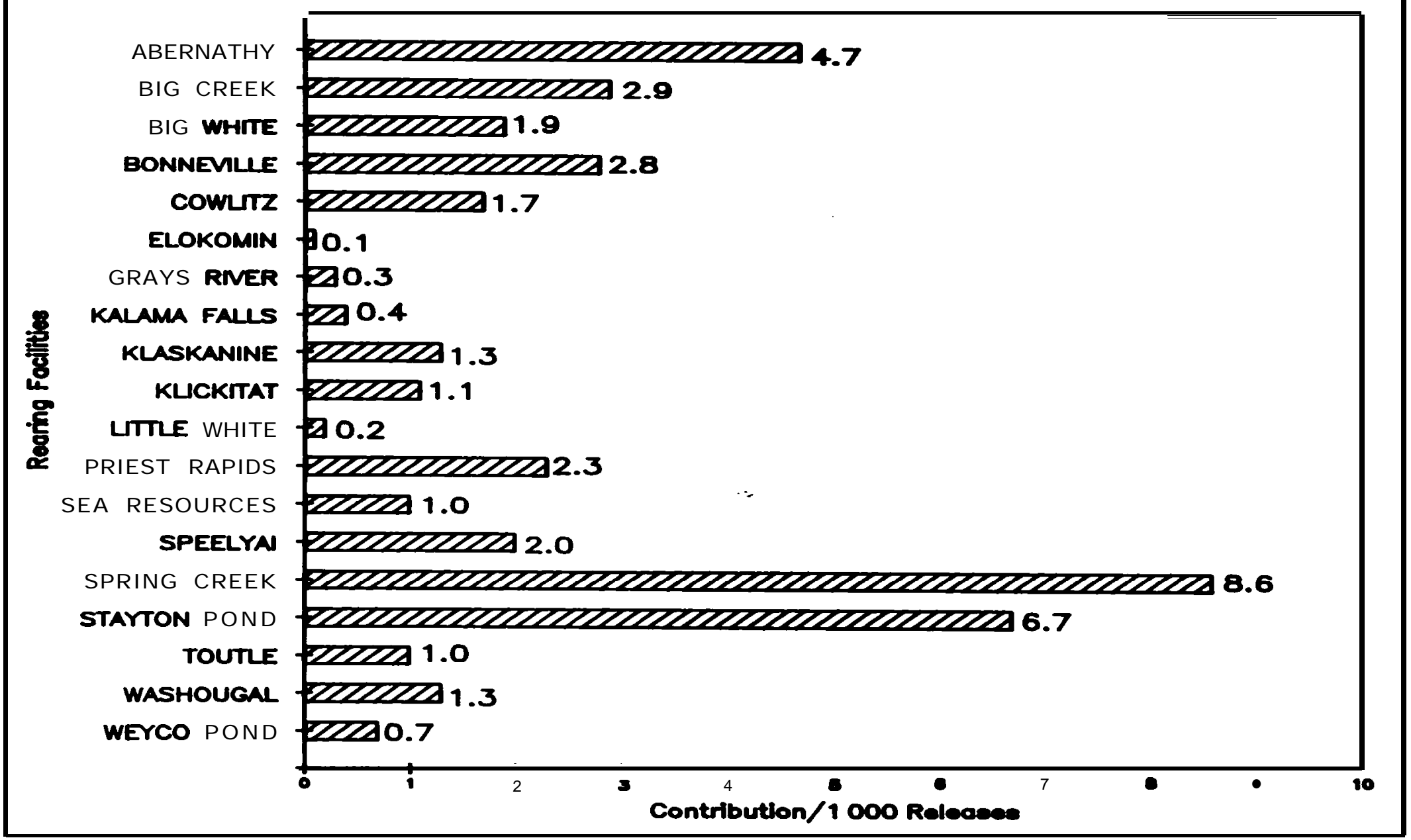


Figure 12.--Fishery contribution of the 1979-brood fall chinook almon from Columbia River rearing facilities by facility.

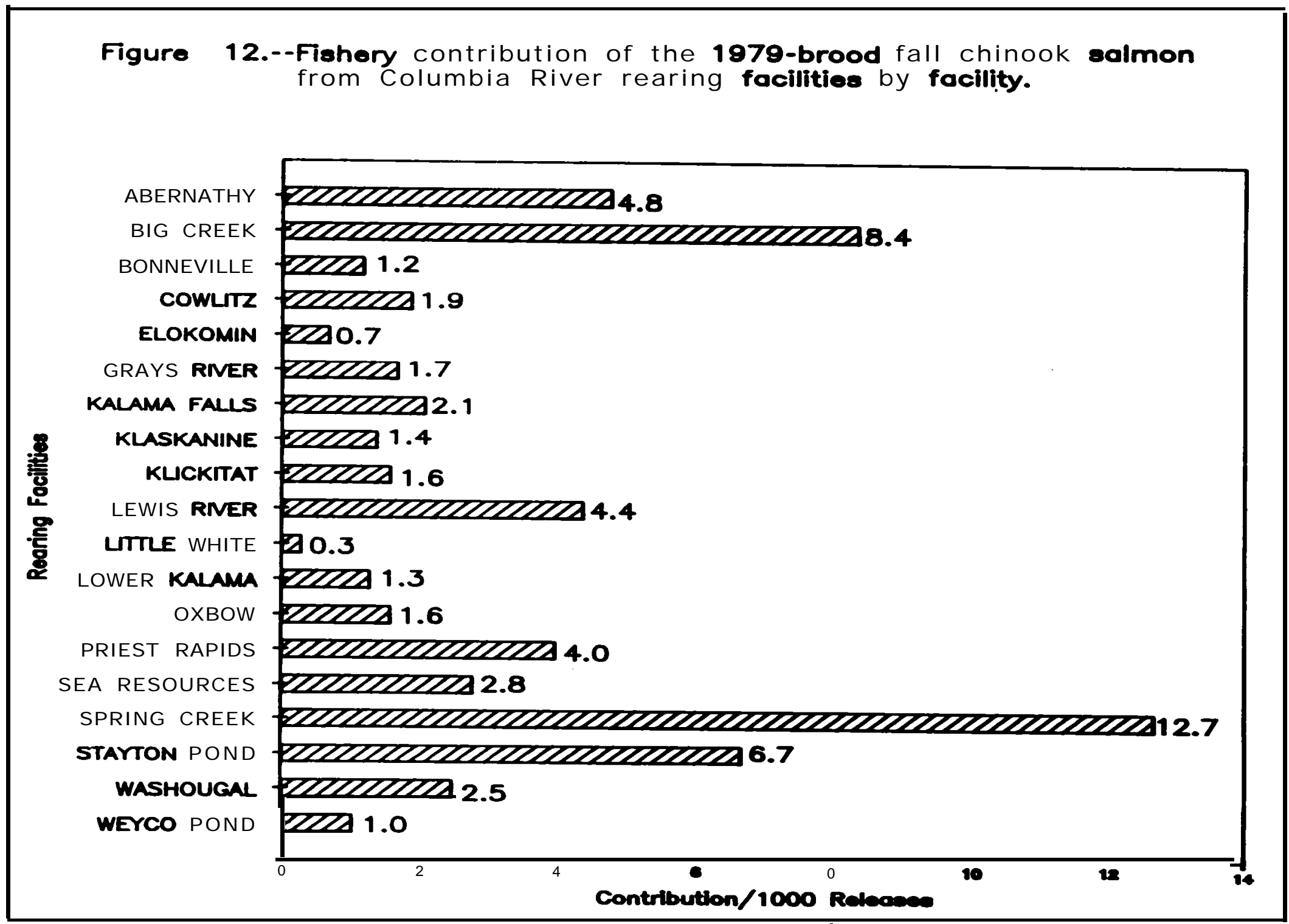


Figure 13.--Fiahery contribution of the 1980-brood fall chinook salmon from Columbia River rearing facilitios by facility.

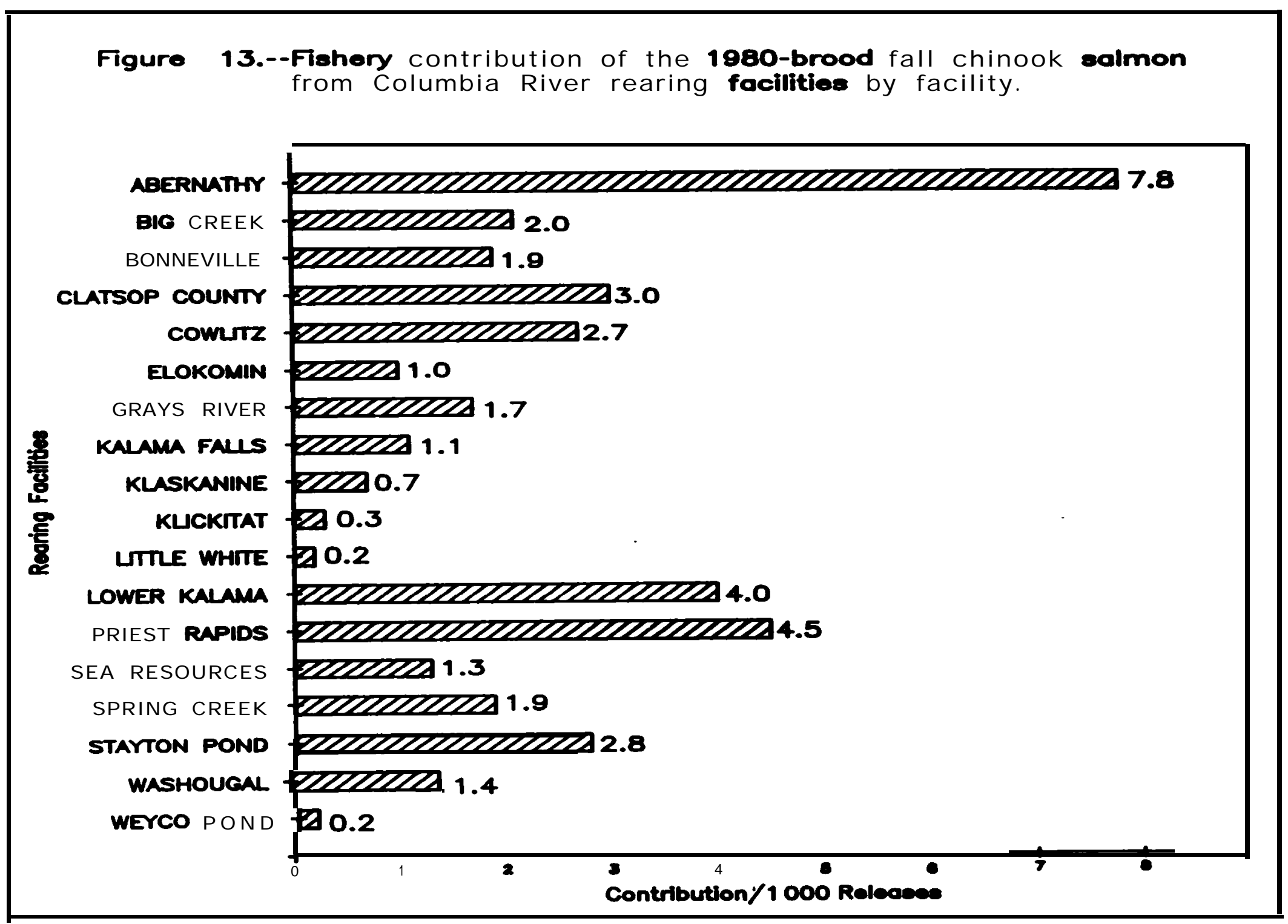


Figure 14.--Fishory contribution of the $\mathbf{1 9 8 1}$-brood fall chinook salmon from Columbia River rearing focilitios by facility.

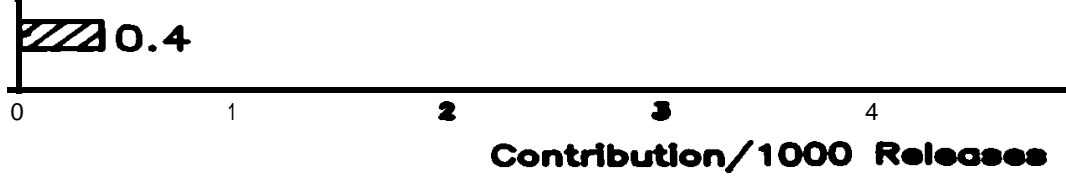


Figure 15.--Proportion of the fishery contribution of 1978-brood fall chinook salmon from Columbia River rearing facilitios attributable to each facility.

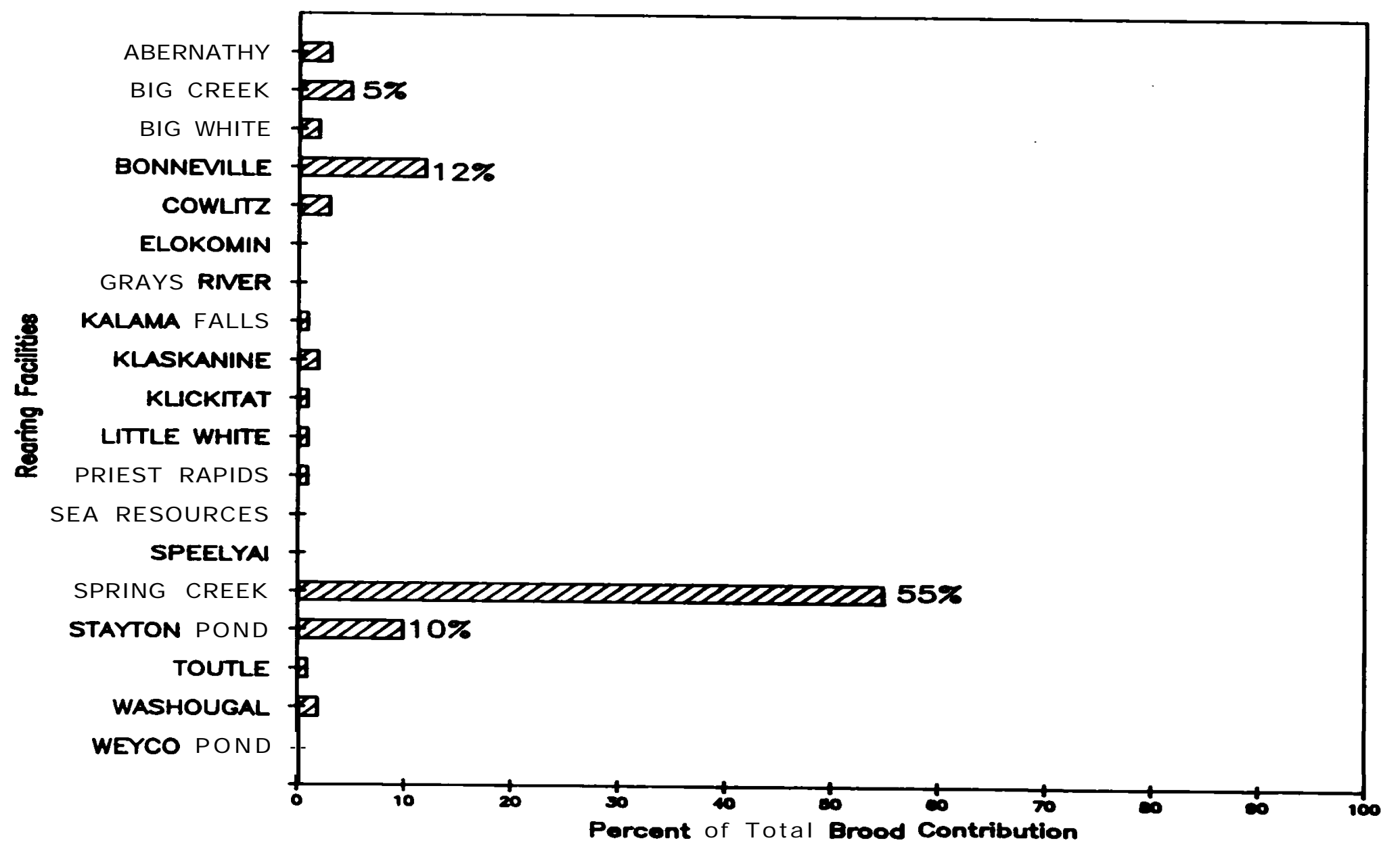




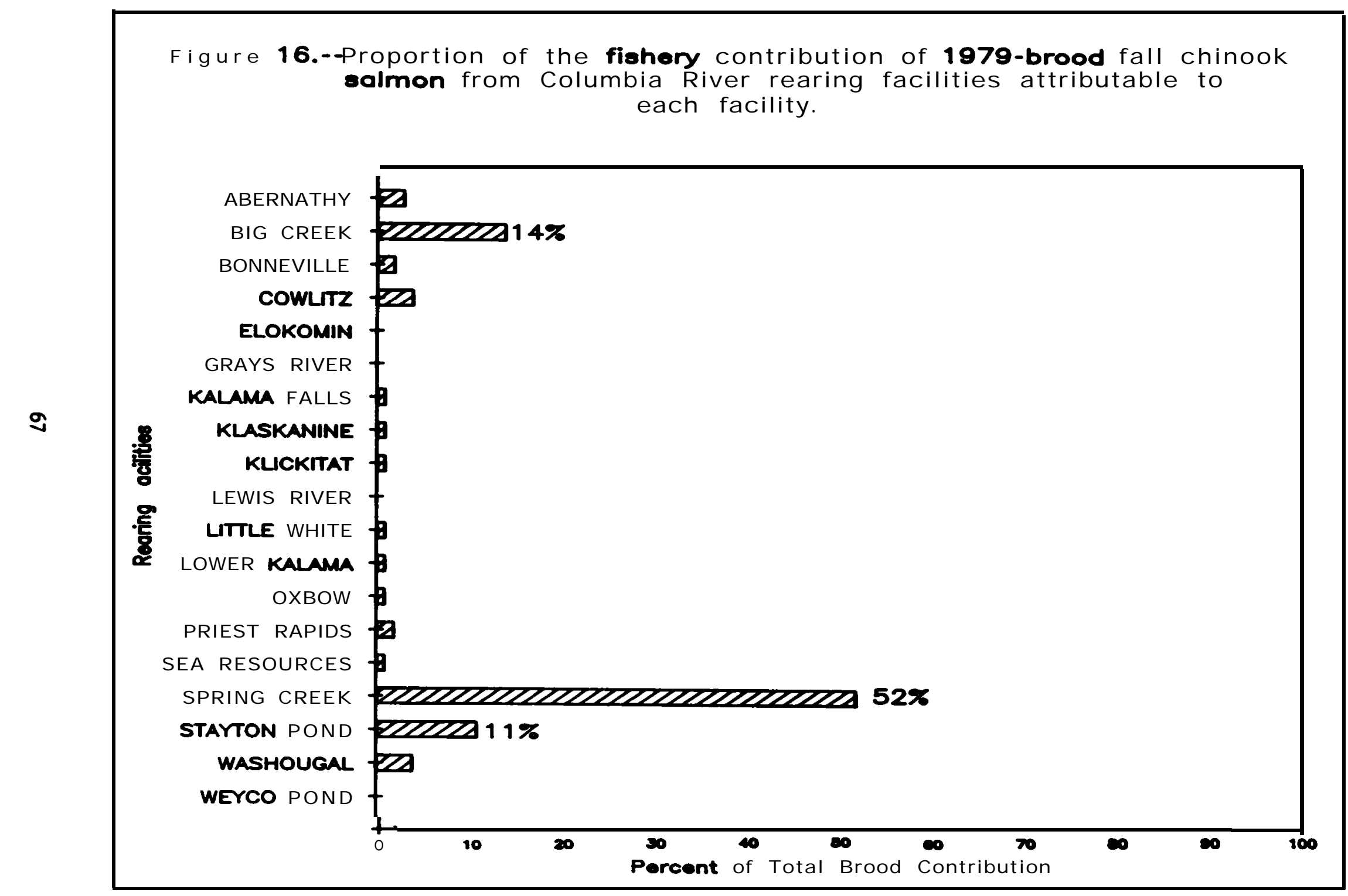


Figure 17.--Proportion of the fishery contribution of $\mathbf{1 9 8 0 - b r o o d ~ f a l l ~ c h i n o o k ~}$ salmon from Columbia River rearing facilities attributable to each facility.

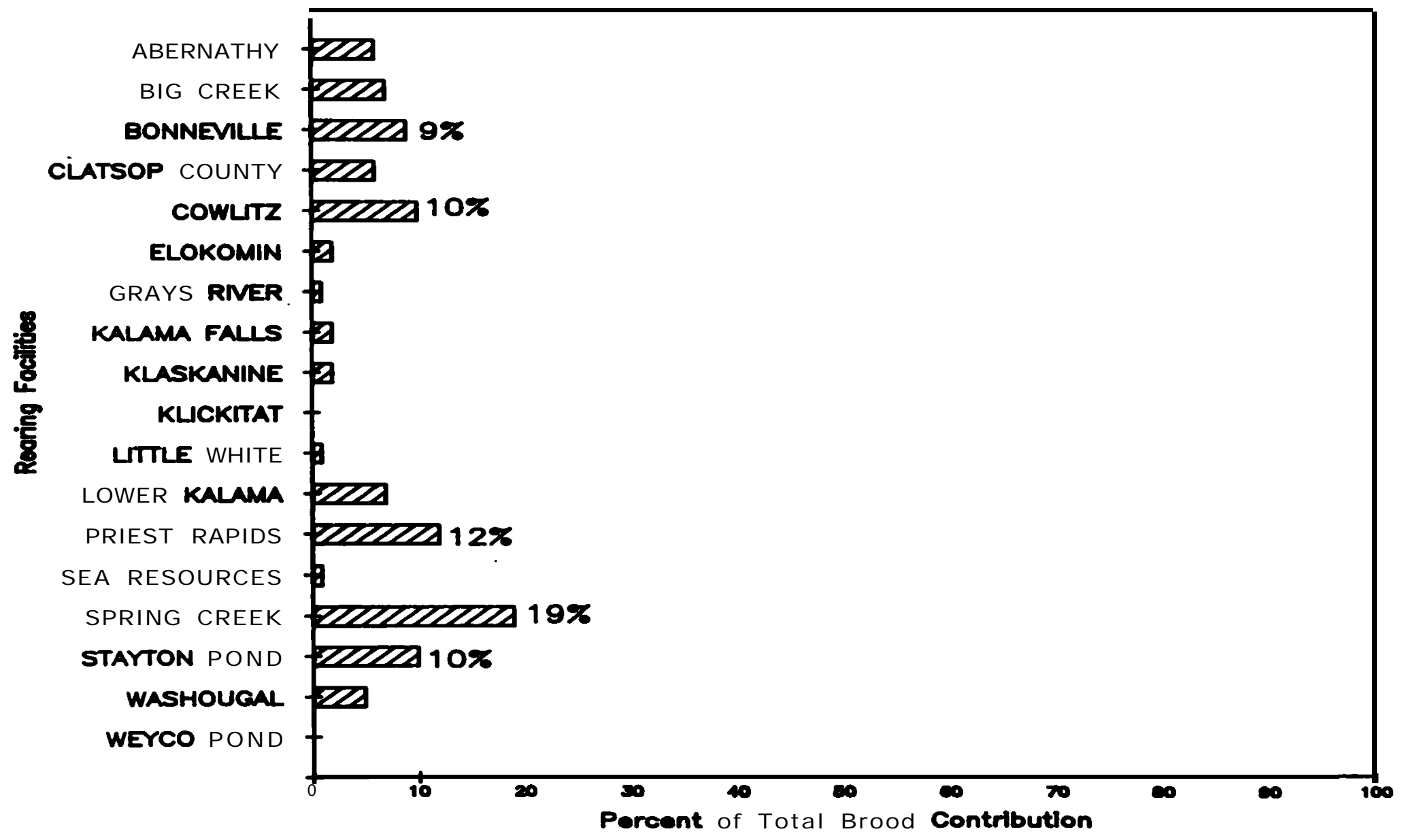


Figure 18.--Proportion of the fishery contribution of $\mathbf{1 9 8 1}$-brood fall chinook almon from Columbia River rearing focilitio attributable to each facility.

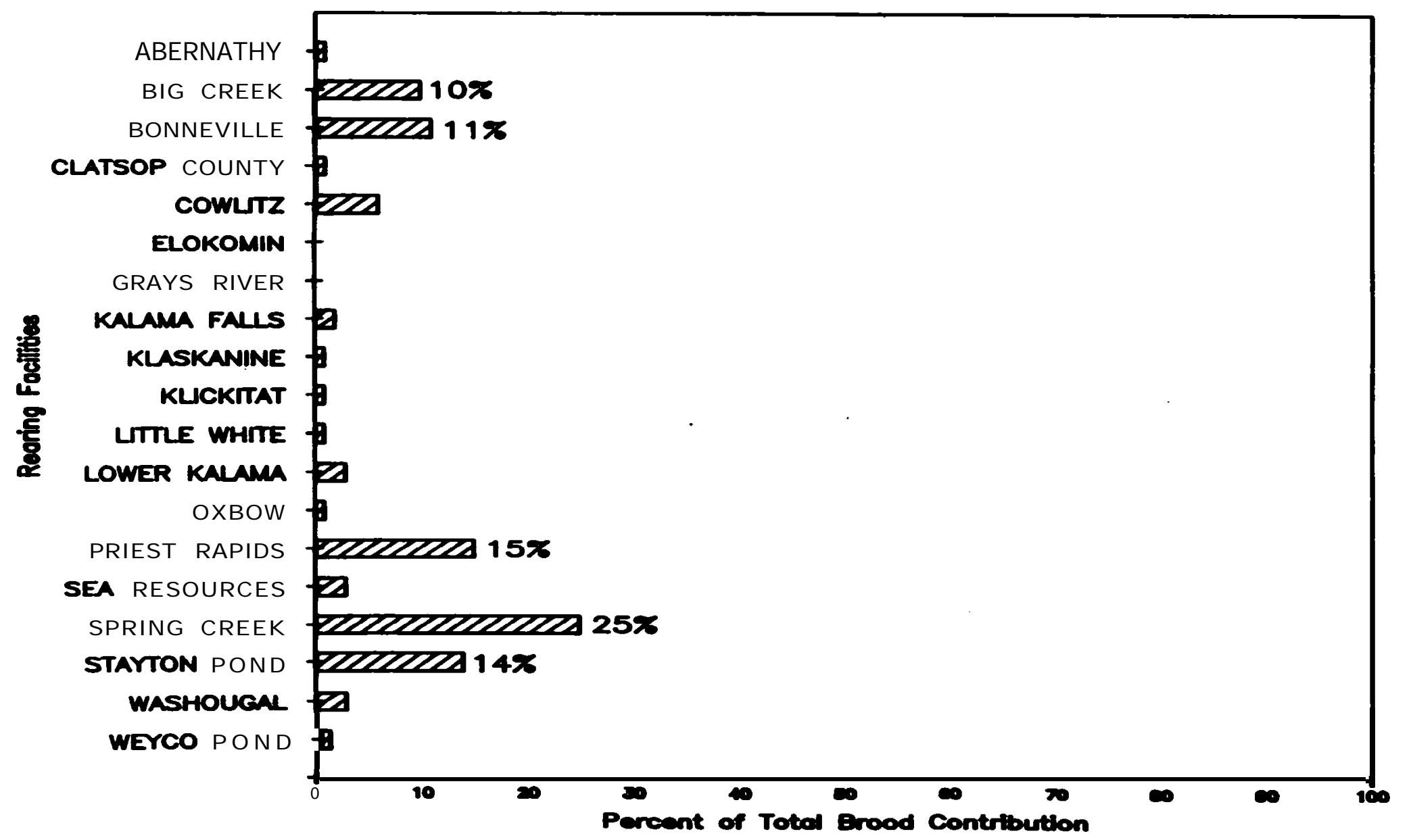


18). Thus the proportions of the flohery contributions of tagged fish from Sprins Creek Hatchery dropped from over 50s to just over 20 over the four brood years, while the proportion of fish in the releases ranged from 16 to $21 \%$.

The proportion of the fishery contribution of fall chinook salmon from Prlese Rapids Hatchery Incresed over the four brood years. The contribution proportion attributable to Priest Rapids Hatchery was is for the 1978 brood (FIgure 15) and increased to 4,12, and 15s for the 1979 through 1981 broods respectively (F1gures 16 - 18). The proportion of the total fish relased from Priest Rapids incraased from 1s (Table 1) to 4, 5, and 5.54 (Tables 2 - 4) for the 1978 through 1981 broods respectively.

All WDF rearing facllities combined show a trend similar to that for Prlest Rapids Hatchery. The 1978 brood tagged fish from WDI facllities represented 428 of the total releases (Table 1). The proportion of total releases from WDF faclilties increased to 54\% for the 1979 brood (Table 2) and then dropped slightly to 52 and S0s for the 1980 and 1981 broods respectively (Tables 3 and 4 ). The proportion of the flshery contribution of eagged fish from WDF facllitles was only 15s for the 1978 brood (Table 9) and Increased to 29, 44, and 41 percent for the 1979 through 1981 broods respectively (Tables 10 - 12).

Rearing Facility and adjacent Stream Reeurns

The age distribution of the tagsed fall chinook salmon at return to the rearing faclilties and adjacent streams appeared to be somowhat different than the age distribution of the fishery contribution. For all brood years combined, the proportions of fish returning by age are: 7v 2-year olds, 46*3-year olds, 41, 4-year olds, and 6s 5-year olds. Only ten tagged 6-year-01d fall chinook salmon were recovered in return sampling. The range of return proportions by brood and age are presented in Fi gure 19. For the 1978 and 1979 broods, the 3-year-old returne comprlsed 50s of the return. For the $1980 \mathrm{brood}$, the 3-year-old and 4-year-old fleheach compr1sed about 408 of the returns. For the 1981 brood, the 4-year-old fish comprised 50s of the return,

When the age of returns are grouped by agency, the resulte also appear to differ from those soon in the fisheries. At USFWS and ODFW facilitien the proportion of 3-year-olds in the return is smaller than the proportion in the f1sher1es. AtWDF facllities the proportion of 4year-old returns slightly $\bullet$ xceeds the proportion in the fisher1es. The proportion of 3 -year-01d returns to USFWS facllities was 65\%, followed by 4-year olds (194), 2-yoar olds (164), and 6-yearolds (0.5s) (Figure 20). AtODW facilities, the proportion of 3-year-old returns was 57\%, followed by 4-year-01ds (398), 2-year-olds (38), and 6-year-olds (18). At WDF facilities, the 4-year-old fish comprised 58. of the return, followed by the 3-year-olds (268), 5-year-olds (128), and 2-year-olds (4). In general, allWDE facilities followed this age at return pattern for all broodyears. The only exceptions, where total returns for a brood were 20 or greater, were Sea Resources Hatchery and Weyco Pond for the 1979 brood and Sea Resources Hatchery for the 1981 brood 
Figure 19.--Proportion of fall chinook salmon from Columbia River rearing facilities escaping the fisheries by age of return and brood year, 1978 - 1981.

3-yer-ald saces

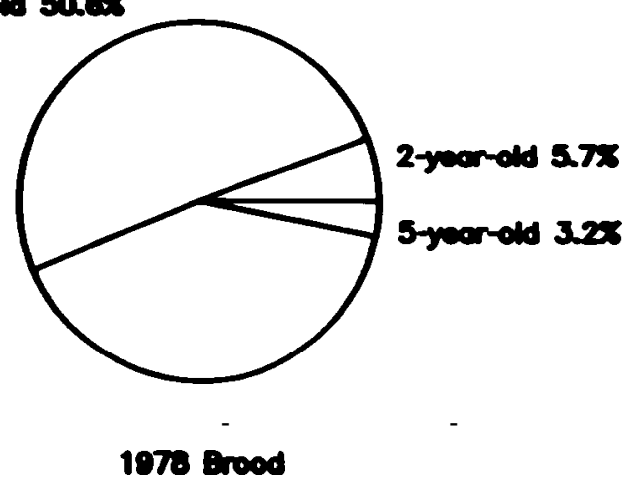

3-yourald 40.45

Hyorold $41 \mathrm{sex}$

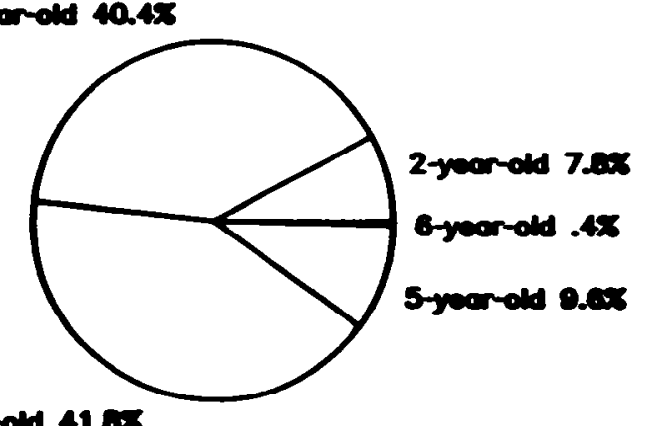

1800 Brood

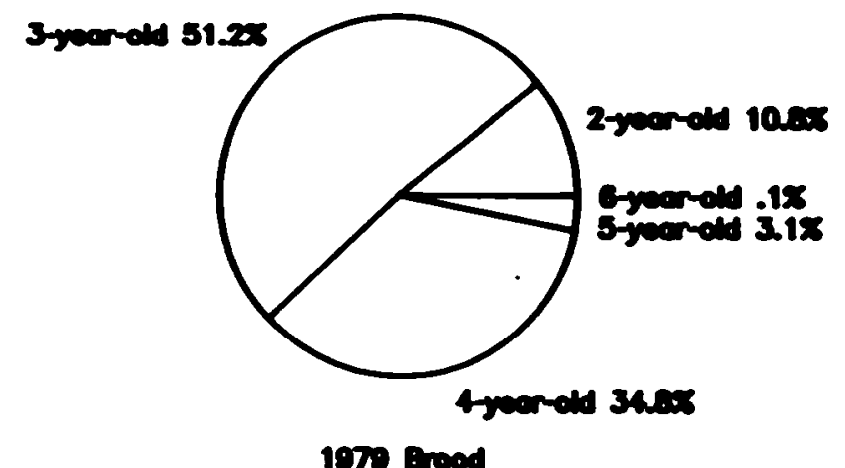

1070 Brood

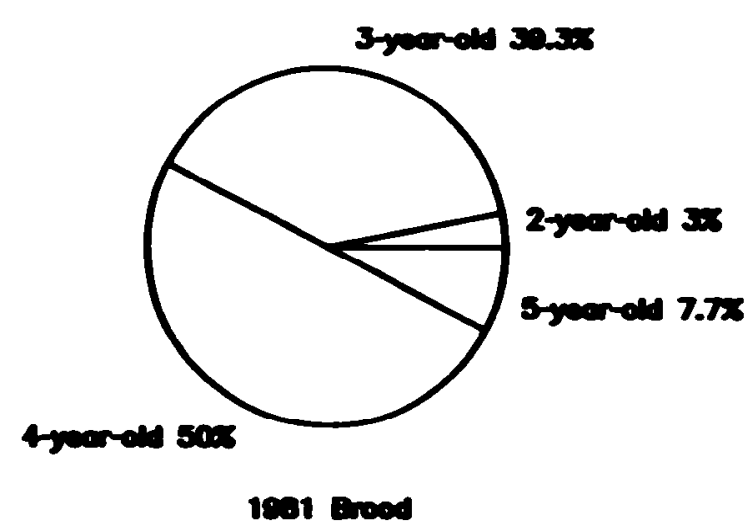


Figure 20.--Proportion of fall chinook salmon from Columbia River rearing focilities escaping the fisheries by age of retum for all brood year-s and focilities combined and by operating agency.

3-yeor-ald 45000

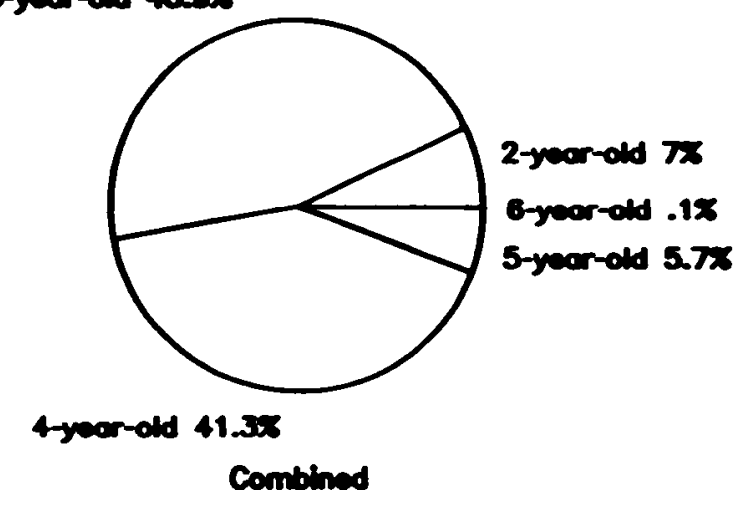

3-yeror-old 56.778

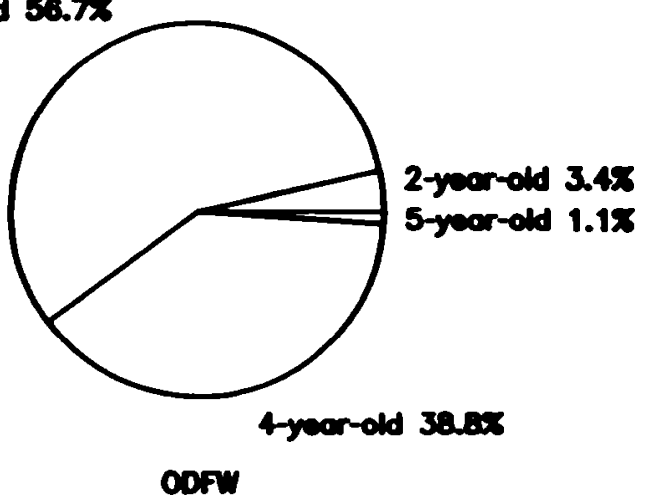

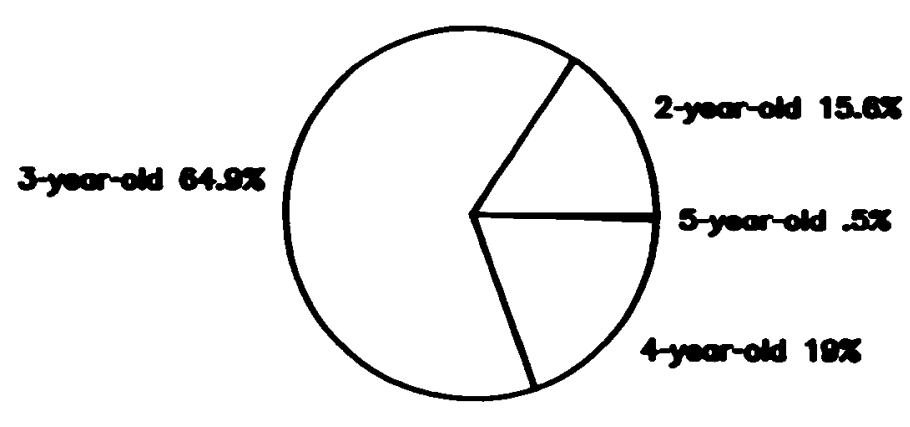

usris

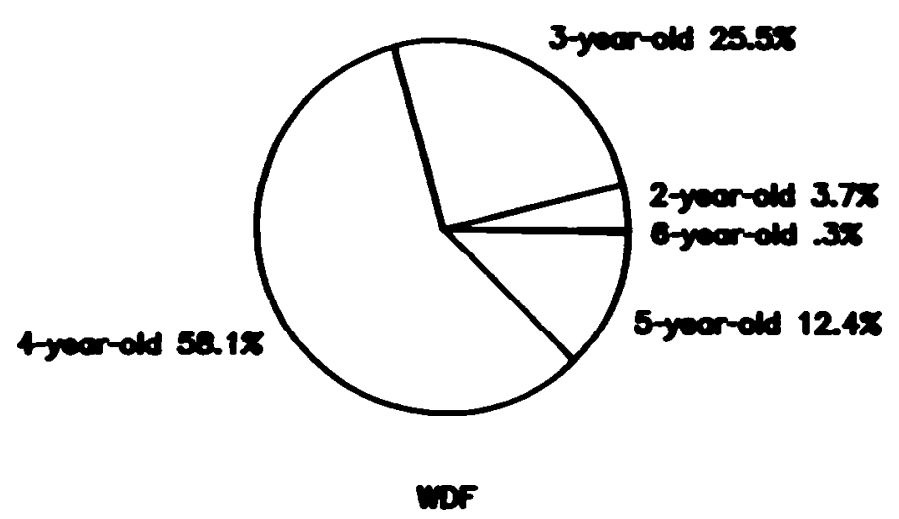


The only i-year-old fish returning were from groups tagged at WDF facilities.

A comparison of the age proportions in the fisheries and at return by rearing facility does not yield a consistent pattern (Appendix Tables 40 - 43). In many cases, there is a greater proportion of older fish (4-. 5-, and 6-year-olds) in the rearing facility and adjacent stream returns. In a few cases, there is a greater proportion of 2- and 3year-old fish in the return. There are very few cases where the fishery recovery proportion and return proportion are relatively close for all age groups.

The extent of straying of returning tagged fish is clearly evident from examination of Appendix Tables 17 through 35. Tagged fall chinook salmon generally strayed to facilities and streams near the release facility. Straying appears to have occurred about equally in all four brood years. Straying appears to be greatest for those facilities on streams emptying into or near the Columbia River estuary. Over 35\% of the fish tagged at Big Creek, Grays River, and Elokonin hatcheries returned to areas other than the release facilities. For Weyco Pond, Klaskanine Hatchery, and Clatsop County ponds, the straying rate was 75 , 85, and 1008 respectively. There is one notable exception to this trend. None of the tagged releases from Sea Resources Hatchery were recovered at sites other than Sea Resources. The straying rates were generally less than 20 and in several cases less than 10 for

facilities upstream of the Columbia River estuary. The one exception to this was Lewis River and Speelyai hatcheries. Straying of fish tagged and released from these facilities to sites outside the Lewis River was 368 for the two facilities combined.

Straying was generally clustered among facilities and streams in the general area of release; estuary influence area (facilities: Abernathy, Big Creek, Clatsop County, Elokomin, Grays River, and Klaskanine), Longview to Portland area (facilities: Cowlitz, Kalama Falls, Lewis River, Lower Kalama, and Toutle), Bonneville Dam area (facilities: Big White, Bonneville, Little White, Spring Creek, and Washougal), and MidColumbia area (Priest Rapids Hatchery). Two exceptions to this trend are tagged releases from Abernathy and Washougal hatcheries. Abernathy Hatchery releases fish into Abernathy Creek below Longview, WA but above the estuary influence. The greatest number of strays from Abernathy were to the Kalama River system in the Longview to Portland area, but Abernathy strays also returned to estuary and Bonneville Dam area facilities. Washougal Hatchery releases fish into the Washougal River, which enters the Columbia River 25 miles below Bonneville Dam. The greatest number of strays from Washougal Hatchery also returned to the Kalana River system. A number of tagged fish from Priest Rapids were recovered at Bonneville Dam, but these fish were intercepted during their upstream migration by a trapping operation in the north-shore fish ladder.

Two returns of tagged fall chinook from this study occurred outside the Columbia River system. A 1979 brood fish from Kalama Falls Hatchery returned to the Deschutes Hatchery on the Deschutes River near Olympia, Washington in 1983 (Appendix Table 23). The Deschutes River empties into the southern end of Puget Sound. A tagged 1980-brood fish from 
Priest Rapids Hatchery returned to Nenah Hatchery on the Nenah River in 1985 (Appendix Table 29). The Nemah River empties into the southern end of Willapa Bay on the Washington coast north of the Columbia River mouth.

In general, the greater the fishery contribution of tagged fall chinook salmon fron a rearing facility, the greater escapement of that group of fish. The fishery contribution to escapement ratio for all broods and all facilities combined is nearly 5 to 1 ; five fish contributed to the fisheries for each fish escaping to a rearing facility or adjacent stream (Table 17). There is a considerable difference among rearing facilities; with the contribution to escapement ranging from 2.3 to 1 for Kalama Falls Hatchery to 19.2 to 1 for Klaskanine Hatchery when all broods are combined. The ranges of contribution to escapement by brood are relatively consistent: 5.3, 5.3, 4.3, and 4.3 to 1 for the 1978 through 1981 broods respectively (Tables 17 - 21). The ranges among rearing facilities, examining brood years separately are 2.0 to 1 for Cowlitz and Kalama Falls hatcheries, 1980 brood (Table 20) and 1981 brood (Table 21) respectively; to fishery contributions but no returns to Klickitat and Sea Resources hatcheries (1978 brood, Table 18).

Total Survival

Total survival in this report is defined as the sum of fishery contribution and returns to rearing facilities and adjacent streams. The average total survival for all brood years and rearing facilities combined is 0.33 (Table 17). The lowest survival was for the 1981 brood (0.258) (Table 21) and the greatest for the 1979 brood (0.468) (Table 19). It is interesting to note that the lowest fishery contribution was for the 1980 brood. Thus the 1980 brood had a lower fishery contribution but appears to have a slightly higher survival than the 1981 brood. The range among rearing facilities, all brood years combined was 0.028 (Little White Hatchery) to 0.78 \% (Spring Creek Hatchery) (Table 17). The range in survivals across brood years and rearing facilities was a low of 0.018 (Elokomin Hatchery, 1978 brood)

(Table 18) to a high of 1.478 (Spring Creek Hatchery, 1979 brood) (Table 19). In general, the greater the fishery contribution for a facility, the greater the total survival.

\section{Benefit/Cost}

The total costs of rearing the 1978 through 1981 broods of fall chinook salmon is estimated at $\$ 6,334,000$. The total benefits derived from the fishery recovers of these fish are estimated at $\$ 36,242,200$ (Tables 22-25). This is an average benefit to cost ratio of 5.7 to 1 . costs increased over the four brood years from $\$ 1.4$ million for the 1978 brood. (Table 22) to $\$ 1.7$ million for the the 1981 brood (Table 25). Benefits ranged from a high of $\$ 14.9$ million for the 1979 brood (Table 23) to a low of $\$ 4.4$ million for the 1981 brood (Table 25). Benefit to cost ratios ranged from a high of 9.8 to 1 for the 1979 brood to a low of 2.5 to 1 for the 1981 brood. 
Table 17.-- Catch, escapement, and survival data for all broods of tagged fall chinook salmon combined $(1978-1981)$ by rearing facility.

\begin{tabular}{|c|c|c|c|c|}
\hline Rearing facility & Catch & Escapement & $\begin{array}{l}\text { Catch/ } \\
\text { escapement }\end{array}$ & $\begin{array}{l}\text { Percent } \\
\text { survival }\end{array}$ \\
\hline ABERNATHY HATCHERY & 2.071 & 487 & $4.3 / 1$ & 0.55 \\
\hline BIG CREEK HATCHERY & 2,670 & 767 & $3.5 / 1$ & 0.53 \\
\hline BIG WHITE POND & 274 & 58 & $4.7 / 1$ & 0.23 \\
\hline BONNEVILLE HATCHERY & 2,211 & 634 & $3.5 / 1$ & 0.30 \\
\hline CLATSOP COUNTY PONDS & 432 & 26 & $16.6 / 1$ & 0.19 \\
\hline COWLITZ HATCHERY & 2,003 & 822 & $2.4 / 1$ & 0.27 \\
\hline ELOKOMIN HATCHERY & 317 & 59 & $5.4 / 1$ & 0.07 \\
\hline GRAYS RIVER HATCHERY & 296 & 65 & $4.6 / 1$ & 0.11 \\
\hline KALAMA FALLS HATCHERY & 704 & 311 & $2.3 / 1$ & 0.15 \\
\hline KLASKANINE HATCHERY & 518 & 27 & $19.2 / 1$ & 0.11 \\
\hline KLICKITAT HATCHERY & 637 & 15 & $42.5 / 1$ & 0.09 \\
\hline LEWIS RIVER-SPEELYAI HATCHERIE & ES 780 & 89 & $8.8 / 1$ & 0.33 \\
\hline LITTLE WHITE HATCHERY & 202 & 38 & $5.3 / 1$ & 0.02 \\
\hline LOWER KALAMA HATCHERY & 1,047 & 317 & $3.3 / 1$ & 0.31 \\
\hline OXBOW HATCHERY & 194 & 31 & $6.3 / 1$ & 0.11 \\
\hline PRIEST RAPIDS HATCHERY & 3,098 & 812 & $3.8 / 1$ & 0.46 \\
\hline SEARESOURCES HATCHERY & 402 & 71 & $5.7 / 1$ & 0.36 \\
\hline SPRING. CREEK HATCHERY & 12,187 & 1,603 & $7.6 / 1$ & 0.78 \\
\hline STAYTON POND & 5,391 & 805 & $6.7 / 1$ & 0.58 \\
\hline TOUTLE HATCHERY & 135 & 41 & $3.3 / 1$ & 0.12 \\
\hline WASHOUGAL HATCHERY & 1.758 & 597 & $2.9 / 1$ & 0.23 \\
\hline WEYCO POND & 276 & 67 & $4.1 / 1$ & 0.05 \\
\hline Total & 37,603 & 7,742 & $4.9 / 1$ & 0.33 \\
\hline
\end{tabular}


Table 18.-- Catch, escapement, and survival data for tagged 1978-brood fall chinook salmon from Columbia River rearing facilities.

\begin{tabular}{|c|c|c|c|c|}
\hline Rearing facility & Catch & Escapement & $\begin{array}{l}\text { Catch/ } \\
\text { escapement }\end{array}$ & $\begin{array}{l}\text { Percent } \\
\text { survival }\end{array}$ \\
\hline ABERNATHY HATCHERY & 532 & 92 & $5.8 / 1$ & 0.56 \\
\hline BIG CREEK HATCHERY & 659 & 186 & $3.5 / 1$ & 0.38 \\
\hline BIG WHITE POND & 274 & 58 & $4.7 / 1$ & 0.23 \\
\hline BONNEVILLE HATCHERY & 854 & 311 & $2.7 / 1$ & 0.38 \\
\hline COWLITZ HATCHERY & 309 & 120 & $2.6 / 1$ & 0.28 \\
\hline ELOKOMIN HATCHERY & 17 & 2 & $8.5 / 1$ & 0.01 \\
\hline GRAYS RIVER HATCHERY & 71 & 20 & $3.6 / 1$ & 0.06 \\
\hline KALAYA FALLS HATCHERY & 91 & 35 & $2.6 / 1$ & 0.06 \\
\hline KLASKANINE HATCHERY & 312 & 15 & $20.8 / 1$ & 0.13 \\
\hline KLICKITAT HATCHERY & 243 & 0 & 0 & 0.11 \\
\hline LITTLE WHITE HATCHERY & 66 & 20 & $3.3 / 1$ & 0.02 \\
\hline PRIEST RAPIDS HATCHERY & 191 & 67 & $2.9 / 1$ & 0.17 \\
\hline SEA RESOURCES HATCHERY & 25 & 0 & 0 & 0.10 \\
\hline SPEELYAI HATCHERY & 325 & 48 & $6.8 / 1$ & 0.24 \\
\hline SPRING CREEK HATCHERY & 5,044 & 795 & $6.3 / 1$ & 1.01 \\
\hline STAYTON POND & 1,884 & 243 & $7.8 / 1$ & 0.75 \\
\hline TOUTLE HATCHERY & 135 & 41 & $3.3 / 1$ & 0.12 \\
\hline WASHOUGAL HATCHERY & 277 & 63 & $4.4 / 1$ & 0.13 \\
\hline WEYCO POND & 65 & 9 & $7.2 / 1$ & 0.08 \\
\hline Total & 11,374 & 2,125 & $5.3 / 1$ & 0.33 \\
\hline
\end{tabular}


Table 19.-- Catch, escapement, and survival data for tagged 1979-brood fall chinook salmon from Columbia River rearing facilities.

\begin{tabular}{|c|c|c|c|c|}
\hline Rearing facility & Catch & Escapement & $\begin{array}{l}\text { Catch/ } \\
\text { escapement }\end{array}$ & $\begin{array}{l}\text { Percent } \\
\text { survival }\end{array}$ \\
\hline ABERNATHY HATCHERY & 713 & 146 & $4.9 / 1$ & 0.58 \\
\hline BIG CREEK HATCHERY & 1,210 & 279 & $4.3 / 1$ & 1.04 \\
\hline BONNEVILLE HATCHERY & 139 & 32 & $4.3 / 1$ & 0.14 \\
\hline COWLITZ HATCHERY & 607 & 166 & $3.7 / 1$ & 0.23 \\
\hline ELOKOMIN HATCHERY & 64 & 13 & $4.9 / 1$ & 0.08 \\
\hline GRAYS RIVER HATCHERY & 65 & 10 & $6.5 / 1$ & 0.20 \\
\hline KALAMA FALL8 HATCHERY & 210 & 86 & $2.4 / 1$ & 0.29 \\
\hline KLASKANINE HATCHERY & 93 & 2 & $46.5 / 1$ & 0.14 \\
\hline KLICKITAT HATCHERY & 249 & 10 & $24.9 / 1$ & 0.16 \\
\hline LEWIS RIVER HATCHERY & 455 & 41 & $11.1 / 1$ & 0.48 \\
\hline LITTLE WHITE HATCHERY & 46 & $\cdot 2$ & $23.0 / 1$ & 0.03 \\
\hline LOWER KALAMA MATCHERY & 185 & 48 & $3.9 / 1$ & 0.16 \\
\hline OXBOW HATCHERY & 159 & 21 & $7.6 / 1$ & 0.18 \\
\hline PRIEST RAPIDS HATCHERY & 438 & 181 & $2.4 / 1$ & 0.42 \\
\hline SEA RESOURCES HATCHERY & 52 & 22 & $2.4 / 1$ & 0.40 \\
\hline SPRING CREEK HATCHERY & 3,646 & 536 & $6.8 / 1$ & 1.47 \\
\hline STAYTON POND & 1,890 & 196 & $9.6 / 1$ & 0.74 \\
\hline WASHOUGAL HATCHERY & 798 & 255 & $3.1 / 1$ & 0.33 \\
\hline WEYCO POND & 94 & 32 & $2.9 / 1$ & 0.13 \\
\hline Total & 11,113 & 2,078 & $5.3 / 1$ & 0.46 \\
\hline
\end{tabular}


Table 20.-. Catch, escapement, and survival data for tagged 1980-brood fall chinook salmon from Columbia River rearing facilities.

\begin{tabular}{|c|c|c|c|c|}
\hline Rearing facility & Catch & Escapement & $\begin{array}{l}\text { Catch/ } \\
\text { escapement }\end{array}$ & $\begin{array}{l}\text { Percent } \\
\text { survival }\end{array}$ \\
\hline ABERNATHY HATCHERY & 641 & 188 & $3.4 / 1$ & 1.00 \\
\hline BIG CREEK HATCHERY & 304 & 95 & $3.2 / 1$ & 0.27 \\
\hline BONNEVILLE HATCHERY & 388 & 160 & $2.4 / 1$ & 0.27 \\
\hline CLATSOP COUNTY PONDS & 349 & 17 & $20 . \mathrm{s} / 1$ & 0.30 \\
\hline COWLITZ HATCHERY & 741 & 369 & $2.0 / 1$ & 0.40 \\
\hline ELOKOKIN HATCHERY & 210 & 36 & $5.8 / 1$ & 0.15 \\
\hline GRAYS RIVER HATCHERY & 140 & 28 & $5.0 / 1$ & 0.23 \\
\hline KALAMA FALLS HATCHERY & 196 & 86 & $2.3 / 1$ & 0.16 \\
\hline KLASKANINE HATCHERY & 68 & 7 & $9.7 / 1$ & 0.07 \\
\hline KLICKITAT HATCHERY & 43 & 4 & $10.8 / 1$ & 0.04 \\
\hline LITTLE WHITE HATCHERY & 56 & 12 & $4.7 / 1$ & 0.03 \\
\hline LOWER KALAMA HATCHERY & 627 & 191 & $3.3 / 1$ & 0.53 \\
\hline PRIEST RAPIDS HATCHERY & 1,021 & 249 & $4.1 / 1$ & 0.54 \\
\hline SEA RESOURCES HATCHERY & 56 & 5 & $11.2 / 1$ & 0.14 \\
\hline SPRING CREEK HATCHERY & 1,950 & 114 & $17.1 / 1$ & 0.40 \\
\hline STAYTON POND & 688 & 88 & $7.8 / 1$ & 0.32 \\
\hline WASHOUGAL HATCHERY & 446 & 196 & $2.3 / 1$ & 0.21 \\
\hline WEYCO POND & 41 & 16 & $2.6 / 1$ & 0.02 \\
\hline Total & 7.965 & 1,861 & $4.3 / 1$ & 0.28 \\
\hline
\end{tabular}


Table 21.-- Catch, escapement, and survival data for tagged 1981-brood fall chinook salmon from Columbia River rearing facilities.

\begin{tabular}{|c|c|c|c|c|}
\hline Rearing facility & Catch & Escapement & $\begin{array}{l}\text { Catch/ } \\
\text { escapement }\end{array}$ & $\begin{array}{l}\text { Percent } \\
\text { survival }\end{array}$ \\
\hline ABERNATHY HATCHERY & 185 & 61 & $3.0 / 1$ & 0.20 \\
\hline BIG CREEK HATCHERY & 497 & 207 & $2.4 / 1$ & 0.54 \\
\hline BONNEVILLE HATCHERY & 830 & 131 & $6.3 / 1$ & 0.31 \\
\hline CLATSOP COUNTY PONDS & 83 & 9 & $9.2 / 1$ & 0.08 \\
\hline COWLITZ HATCHERY & 346 & 167 & $2.1 / 1$ & 0.17 \\
\hline ELOKOMIN HATCHERY & 26 & 8 & $3.3 / 1$ & 0.03 \\
\hline GRAYS RIVER HATCHERY & 20 & 7 & $2.9 / 1$ & 0.04 \\
\hline KALAMA FALLS HATCHERY & 207 & 104 & $2.0 / 1$ & 0.18 \\
\hline KLASKANINE HATCHERY & 45 & 3 & $15 / 1$ & 0.05 \\
\hline KLICKITAT HATCHERY & 102 & 1 & $102 / 1$ & 0.10 \\
\hline LITTLE WHITE HATCHERY & 34 & 4 & $8.5 / 1$ & 0.02 \\
\hline LOWER KALAMA HATCHERY & 235 & 78 & $3.0 / 1$ & 0.22 \\
\hline OXBOW HATCHERY & 35 & 10 & $3.5 / 1$ & 0.04 \\
\hline PRIEST RAPIDS HATCHERY & 1,448 & 315 & $4.6 / 1$ & 0.57 \\
\hline SEA RESOURCES HATCHERY & 269 & 44 & $6.1 / 1$ & 0.70 \\
\hline SPRING CREEK HATCHERY & 1,547 & 158 & $9.8 / 1$ & 0.43 \\
\hline STAYTON POND & 929 & 278 & $3.3 / 1$ & 0.45 \\
\hline WASHOUGAL HATCHERY & 237 & 83 & $2.9 / 1$ & 0.19 \\
\hline WEYCO POND & 76 & 10 & $7.6 / 1$ & 0.04 \\
\hline Total & 7,151 & 1.678 & $4.3 / 1$ & 0.25 \\
\hline
\end{tabular}


Table 22.- Cost of rearing and benefits for the 1978-brood fall chinook salmon at Columbia River rearing facilities.

\begin{tabular}{|c|c|c|c|}
\hline Rearing facility & Rearing costs & 'Benefits & $\begin{array}{c}\text { Benefit/Cost } \\
\text { ratio }\end{array}$ \\
\hline ABERNATHY HATCHERY & 99,915 & 262,800 & $2.6 / 1$ \\
\hline BIG CREEKHATCHERY & 58,424 & 611,100 & $10.4 / 1$ \\
\hline BIG WHITE POND & 28,851 & 228,500 & $7.9 / 1$ \\
\hline BONNEVILLE HATCHERY & 195,468 & $1,459,900$ & $7.5 / 1$ \\
\hline COWLITZ HATCHERY & $65,339^{2}$ & 356,700 & $5.5 / 1$ \\
\hline ELOKOMIN HATCHERY & 26,008 & 9,600 & $0.4 / 1$ \\
\hline GRAYS RIVER HATCHERY & 33,048 & 22,900 & $0.7 / 1$ \\
\hline KAIAMA FALIS HATCHERY & 74,834 & 66,600 & $0.9 / 1$ \\
\hline KLASKANINE HATCHERY & 55,292 & 178,900 & $3.2 / 1$ \\
\hline KIICKITAT HATCHERY & 50,010 & 134.800 & $2.7 / 1$ \\
\hline LITTLE WHITE HATCHERY & 217,740 & 43,300 & $0.2 / 1$ \\
\hline PRIEST RAPIDS HATCHERY & 67,443 & 77.900 & $1.2 / 1$ \\
\hline SEA RESOURCES HATCHERY & $15.165^{3}$ & 21.300 & $1.4 / 1$ \\
\hline SPRING CREEK HATCHERY & 252,614 & $6,179,400$ & $24.5 / 1$ \\
\hline STAYTON POND & 78,076 & $1,171,100$ & $15.0 / 1$ \\
\hline TOUTLE HATCHERY & 22,689 & 66,800 & $2.9 / 1$ \\
\hline
\end{tabular}


Table 22... (Continued)

\begin{tabular}{crrr}
\hline Rearing facility & Rearing costs & Benefits & $\begin{array}{c}\text { Benefit/Cost } \\
\text { ratio }\end{array}$ \\
\hline WASHOUGAL HATCHERY & 74,202 & 220,600 & $3.0 / 1$ \\
WEYCO POND & 17,016 & 7,500 & $0.4 / 1$ \\
\hline Total & $\$ 1,432,134$ & $\$ 11,187,600$ & $7.8 / 1$ \\
\hline
\end{tabular}

1 Includes: food, chemicals and drugs, labor, overhead, transportation, supplies, equipment. power, maintenance.

2 Power cost estimated as an average of other WDF facilities. chinook salmon at Columbia River rearing facilities.

3 Cost estimated as an average cost for fish released at WDF facilities.

4 Labor cost estimated as a ratio of the average food cost to labor cost at other WDF facilities. 


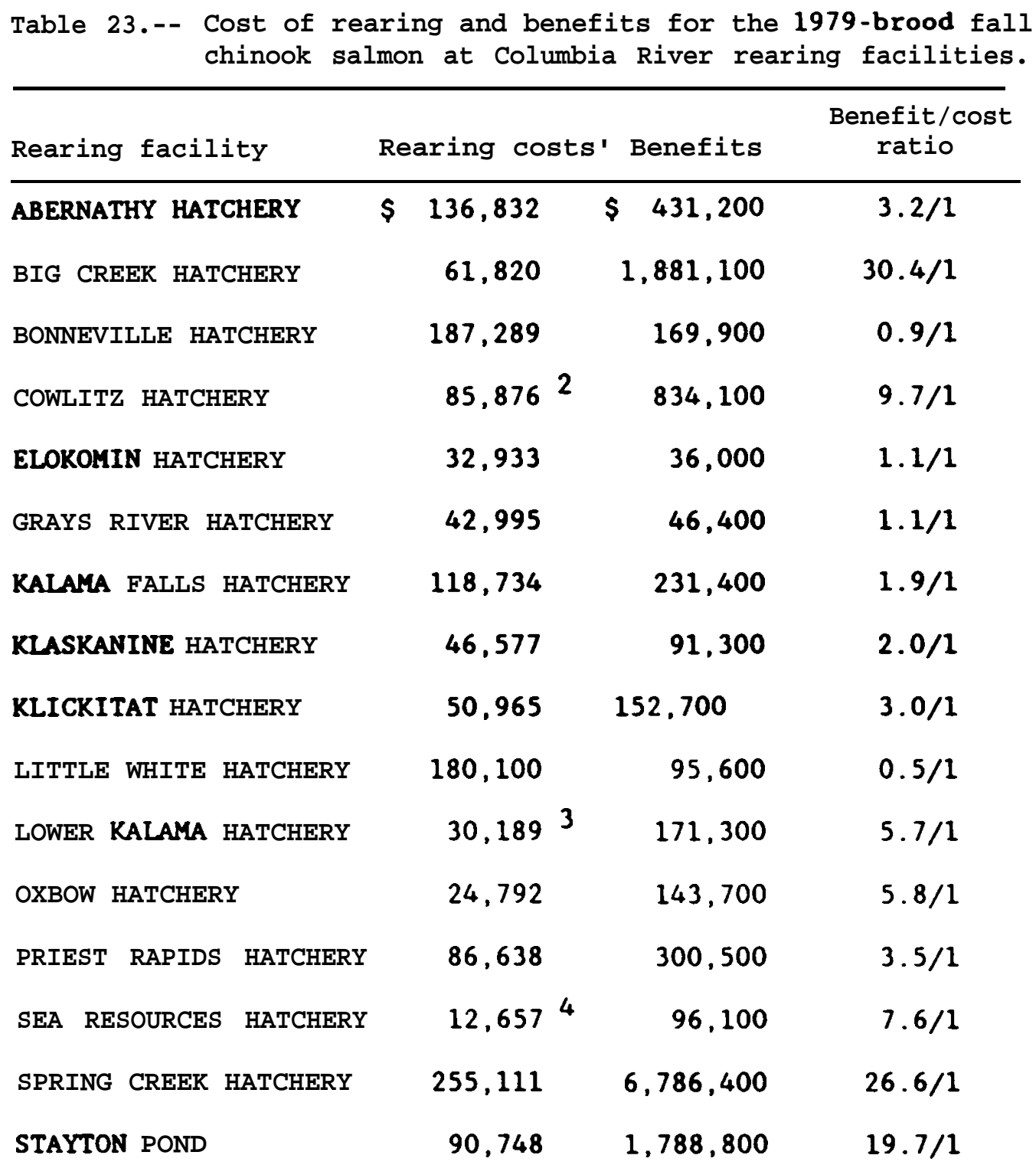


Table 23. - (Continued)

\begin{tabular}{crrc}
\hline Rearing facility & Rearing $\operatorname{costs}^{1}$ Benefits & $\begin{array}{c}\text { Benefit/cost } \\
\text { ratio }\end{array}$ \\
\hline WASHOUGAL HATCHERY & 56,814 & 613,700 & $10.8 / 1$ \\
WEYCO POND & 22,725 & 66,500 & $2.9 / 1$ \\
\hline Total & $\$ 1,523,795$ & $\$ 14,874,700$ & $9.8 / 1$ \\
\hline
\end{tabular}

1 Includes: food, chemicals and drugs, labor, overhead, transportation, supplies, equipment, power, maintenance.

2 Power cost estimated as an average of other WDF facilities.

3 Rearing cost estimated from the ratio of food cost at Lower Kalama Hatchery and total food cost at all other WDF hatcheries to rearing cost at Lower Kalama and total rearing cost at all other WDF hatcheries.

4 Cost estimated as an average cost for fish released at WDF facilities.

5 Labor cost estimated as a ratio of the average food cost to labor cost at other WDF facilities. 


\begin{tabular}{|c|c|c|c|}
\hline Rearing facility & Rearing costs' & ' Benefits & $\begin{array}{c}\text { Benefit/cost } \\
\text { ratio }\end{array}$ \\
\hline ABERNATHY HATCHERY & 111,196 & 426,400 & $3.8 / 1$ \\
\hline BIG CREEK HATCHERY & 67,471 & 379,700 & $5.6 / 1$ \\
\hline BONNEVILLE HATCHERY & 168,098 & 569,500 & $3.4 / 1$ \\
\hline CLATSOP COUNTY PONDS & 31,316 & 300,200 & $9.6 / 1$ \\
\hline COWLITZ HATCHERY & $91,480^{2}$ & 607,700 & $6.6 / 1$ \\
\hline ELOKOMIN HATCHERY & 38,580 & 77,600 & $2.0 / 1$ \\
\hline GRAYS RIVER HATCHERY & 38,187 & 62,400 & $1.6 / 1$ \\
\hline KALAMA FALLS HATCHERY & 141,898 & 154,900 & $1.1 / 1$ \\
\hline KLASKANINE HATCHERY & 51,818 & 44,000 & $0.8 / 1$ \\
\hline KLICKITAT HATCHERY & 59.703 & 20.500 & $0.3 / 1$ \\
\hline LITTLE WHITE HATCHERY & 185,900 & 74,500 & $0.4 / 1$ \\
\hline LOWER KALAMA HATCHERY & $17.847^{3}$ & 470,600 & $26.4 / 1$ \\
\hline PRIEST RAPIDS HATCHERY & 121,093 & 558,800 & $4.6 / 1$ \\
\hline SEA RESOURCES HATCHERY & $15.832^{4}$ & 27.700 & $1.8 / 1$ \\
\hline SPRING CREEK HATCHERY & 258,360 & 978,200 & $3.8 / 1$ \\
\hline STAYTON POND & 72,731 & 668,400 & $9.2 / 1$ \\
\hline
\end{tabular}


Table 24. - (Continued)

\begin{tabular}{crrc}
\hline \hline Rearing facility & Rearing costs' & Benefits & $\begin{array}{c}\text { Benefit/cost } \\
\text { ratio }\end{array}$ \\
\hline WASHOUGAL HATCHERY & 106,096 & 333,600 & $3.1 / 1$ \\
WEYCO POND & 52,299 & 43,500 & $0.8 / 1$ \\
\hline Total & $\$ 1,629,905$ & $\$ 5,805,100$ & $3.6 / 1$ \\
\hline
\end{tabular}

1 Includes: food, chemicals and drugs, labor, overhead, transportation, supplies, equipment, power, maintenance.

2 Power cost estimated as an average of other WDF facilities.

3 Rearing cost estimated from the ratio of food cost at Lower Kalana Hatchery and total food cost at all other WDF hatcherles to-rearing cost at Lower Kalama and total rearing coat at all other UDF hatcheries.

4 Cost estimated as an average cost for fish released at WDF facilities.

5 Labor cost estimated as a ratio of the average food cost to labor cost at other WDF facilities. 
Table 25.- Cost of rearing and benefits for the 1981-brood fall chinook salmon at Columbia River rearing facilities.

\begin{tabular}{|c|c|c|c|}
\hline Rearing facility & Rearing & Benefits & $\begin{array}{c}\text { Benefit/costs } \\
\text { ratio }\end{array}$ \\
\hline ABERNATHY HATCHERY & 117,602 & 75,000 & $0.6 / 1$ \\
\hline BIG CREEKHATCHERY & 70,104 & 531,300 & $7.6 / 1$ \\
\hline BONNEVILLE HATCHERY & 220,997 & 491,100 & $2.2 / 1$ \\
\hline CLATSOP COUNTY PONDS & 25,194 & 39,100 & $1.6 / 1$ \\
\hline COWLITZ HATCHERY & $118,998^{2}$ & 318,100 & $2.7 / 1$ \\
\hline ELOKOMIN HATCHERY & 39,153 & 18,400 & $0.5 / 1$ \\
\hline GRAYS RIVER HATCHERY & 22,324 & 7,200 & $0.3 / 1$ \\
\hline KAIAMA FALLS HATCHERY & 147,882 & 122,400 & $0.8 / 1$ \\
\hline KLASKANINE HATCHERY & 45,653 & 22,900 & $0.5 / 1$ \\
\hline KLICKITAT HATCHERY & 63,367 & 47,900 & $0.8 / 1$ \\
\hline LITTLE WHITE HATCHERY & 242,100 & 29,600 & $0.1 / 1$ \\
\hline LOWER KALAMA HATCHERY & $29.755^{3}$ & 143,600 & $4.8 / 1$ \\
\hline OXBOW HATCHERY & 30,973 & 48,200 & $1.6 / 1$ \\
\hline PRIEST RAPIDS HATCHERY & 119,884 & 489.600 & $4.1 / 1$ \\
\hline SEA RESOURCES HATCHERY & $15,005^{4}$ & 102,500 & $6.8 / 1$ \\
\hline SPRING CREEK HATCHERY & 267,625 & $1,084,000$ & $4.1 / 1$ \\
\hline STAYTON POND & 75,819 & 749,000 & $9.9 / 1$ \\
\hline
\end{tabular}


Table 25.-- (Continued)

\begin{tabular}{crrc}
\hline Rearing facility & Rearing costs' & Benefits & $\begin{array}{c}\text { Benefit/costs } \\
\text { ratio }\end{array}$ \\
\hline WASHOUGAL HATCHERY & 57,017 & 109,400 & $1.9 / 1$ \\
WEYCO POND & 38,750 & 40,300 & $1.0 / 1$ \\
\hline Total & $\$ 1,748,202$ & $\$ 4,374,800$ & $2.5 / 1$ \\
\hline
\end{tabular}

1 Includes: food, chemicals and drugs, labor, overhead, transportation, supplies, equipment, power, maintenance.

2 Power cost estimated as an average of other WDF facilities.

3 Rearing cost estimated from the ratio of food cost at Lower Kalama Hatchery and total food cost at all other WDF hatcharies to rearing cost at Lower Kalama and total rearing cost at all other WDF hatcheries.

4 Cost estimated as an average cost for fish released at WDF facilities.

5 Labor cost estimated as a ratio of the average food cost to labor cost at other WDF facilities. 
By rearing facility across all broods, the benefits ranged from a a high of $\$ 6.8$ million for the 1979-brood fall chinook salmon from Spring Creek Hatchery to a low of $\$ 7,200$ for the 1981-brood fish from Grays River Hatchery. In general, benefits increased as fishery contribution increased.

By rearing facility over the four brood years, benefit to cost ratios ranged from a high of 26.6 to 1 for the 1979-brood fish from spring Creek Hatchery to a low of 0.1 to 1 for the 1981-brood from Little White Hatchery. Benefit to cost ratios were less than one for at least one year at 25 (18 of 73) of the rearing facilities over the four brood years. Little White Hatchery was the only facility with a less the 1 to 1 ratio for all four brood years. Elokonin, Grays River, Kalana Falls, Klaskanine, and Klickitat hatcheries and Weyco Pond had a negative ratio for two brood years.

\section{DISCUSSION}

Downstresm Migrant Recoveries

One of the objectives of the sampling of the downstresm migrants in the Columbia River estuary was to provide capture proportions which could be related to adult survival (Dawley et al. 1986). Estuarine recoveries do not directly represent numbers of fish in the river, but rather vary due to factors affecting catch efficiency: fish size, release location and date, river flow, and survival to the point of recovery. In previous studies, comparisons of estuarine recovery percentages among marked groups have been used successfully to identify survival changes during riverine migration resulting from treatment differences among similar groups (i.e. size at release for yearling fish and stock differences. The survival differences to the estuary in these cases correlated with adult survival data (Dawley et al. 1986). However, for these subyearling fall chinook salmon, estuarine recoveries were generally not an indicator of adult survival. There are several reasons for this. First, there were too few replicate releases to adequately compare juvenile versus adult recoveries. Second, there are no treatment comparisons which are appropriate for juvenile versus adult comparisons because of differences in fish size at release, and release date and location. Third, the duration of the study was insufficient to develop a model for expected survivals to the estuary which could be compared to new recovery proportions for identifying abnormal survival to the estuary in relation to adult recovery differences. Finally, adult recovaries can be strongly influenced by unknown conditions during ocean residence.

Recoveries of tagged fall chinook salmon from Priest Rapids Hatchery at McNary and John Day dams are only indicative that the tag groups were migrating downstream after release. Comparisons of recovery numbers at different locations are not valid because of differences in fish guidance efficiency at the dams and sampling effort at the different recovery locations. 
Fishery and Age Distribution of Catch

During the study with 1961- through 1964-brood fall chinook salmon from Columbia River hatcheries, the average distribution of fishery recoveries was similar to the distribution of the 1979-brood fish. The greatest proportion of the recoveries for the 1961 - 1964 broods was to the Washington fisheries (38.1\%). followed by British Columbia (33.7\%), and Columbia River (23.1\%). The British Columbia recovery proportion ranged from 27 to 39\% for all 1961- through 1964- brood fish. The Washington recovery proportion ranged from 33 to $39 \%$ (Wahle and Vreeland 1978). In the present study, the British Columbia recovery proportion ranges from 33 to $53 \%$ while the Washington recovery proportion ranges from 8 to $37 \%$. The British Columbia recovery proportions equal or exceed $50 \%$ for the 1980 and 1981 broods. The Washington recovery proportions decreased dramatically from 37\% for the 1979 brood to 21 and $8 \%$ for the 1980 and 1981 broods respectively.

Fall chinook salmon from Spring Creek Hatchery have typically contributed most heavily to the Washington fisheries (Wahle and Vreeland 1978). This was also the case for the 1978 and 1979 broods $(37$ and 39\% respectively). For the 1980 and 1981 broods, the fall chinook from Spring Creek contributed most heavily to the British Columbia fisheries (44 and 43\% respectively) (Appendix Tables 1 - 4). Thus there appears to be a northerly shift in fishery recoveries during this study.

A factor in the apparent more northerly distribution may be the 1982 1983 El Nino (unusually warm sea surface temperatures in the northeast Pacific Ocean) (Hayes and Henry 1985). The El Nino would have effected the 1980-brood fall chinook in their second and third years of life and the 1981 brood in their first and second years. The warmer water moving up from South America may have pushed some of the fish farther to the north.

Another factor in the apparent northerly shift in recovery distribution is the changes that were occurring in the fishing seasons during the later years of this study (1984 - 1986). As a result of the Pacific Salmon Treaty between Canada and the United States, time and area closures in fisheries were instituted to restrict recoveries of certain stock of chinook salmon. Quotas for marine catches of chinook salmon were established by fishery managers in Alaska, British Columbia, Washington, and Oregon in 1984 (Anon. 1984). The quota established for 1984 in the west coast Vancouver Island troll fishery was equal to that for 1983. The length of the Georgia Strait troll fishery was reduced from April 15 - September 30 in 1983 to July 1 - August 31 in 1984. The west coast Vancouver Island and Georgia Strait fisheries are the primary locations of recovery in British Columbia of fall chinook salmon from the Columbia River. Despite the quotas and changes in fishing time, the British Columbia catch of chinook salmon in 1984 exceeded the 1983 catch and the 1984 quotas. Neither the catch or the effort in the British Columbia fisheries was reduced by the regulation changes (Anon. 1984). The quotas for ocean chinook fisheries in 1984 severely restricted catches off Washington and Oregon. This was done to protect depressed fall chinook salmon stocks in the Columbia River and coho salmon stocks 
in Washington coastal tributaries. The 1984 troll catch of chinook salmon in Washington was $40 \%$ of the 1983 catch (Anon. 1984).

The fishery changes in 1984 effected the 4-year-old fall chinook salmon from the 1980 brood and the 3-year-old fish from the 1981 brood. The 3and 4-year-olds comprise 85 - 90\% of the catches of fall chinook salmon from Columbia River rearing facilities. Thus the reduction in catch in Washington and Oregon fisheries and the increase in catch in the British Columbia fisheries is a likely factor in the apparent northern shift of catch distribution during this study.

The proportion of the recovery in the Alaska fisheries also increased from the 1978 to the 1980 and 1981 broods. The Alaska proportions of the recovery were $1.2,3.1,6.6$, and 4.8 for the 1978 through 1981 broods respectively (Figure 7). Potential reasons for the increase in Alaska recovery for the later brood years are the $\mathrm{El}$ Nino and changes in the Alaska marine fisheries in 1984 and 1985. The length of the comercial troll fishing season in Southeast Alaska has been reduced from 149 days in 1980 to 41 days in 1986 (Davis and Seibel 1989). The reduced fishing seasons were achieved by concentrating the fishing in the sumeez months (June, July, and August) rather than spreading the season out from April to September. The over $70 \%$ reduction in fishing . time resulted in only a 30\% reduction in catch (Davis and Seibel 1989). The reduced fishing time may have increased the fishing effort on fall chinook salmon from Columbia River rearing facilities.

The Alaska recoveries of fall chinook salmon from Columbia River facilities came almost exclusively from WDF facilities. Only two nonUDF facilities (Sea Resources Hatchery, 1979 brood and Abernathy Hatchery, 1980 brood) contributed fish to the Alaska fisheries (Appendix Tables 2 and 3 ). Both of these facilities are located on Washington tributaries to the Columbia River. It is difficult to compare this trend with recoveries in the 1961- through 1964-brood study. Fishery sampling in the Alaska fisheries did not take place during all of the seven recovery years for the earlier study, and all hatcheries were not uniquely identified with a mark each year of the study. However, the only hatcheries known to contribute fish to the Alaska fisheries during the 1961 - 1964 study were Kalama Falls and Lower Ralama (WDF facilities) (Wshle and Vreeland 1978).

The primary reason for the change in age distribution among the broods is the contribution of fall chinook salmon from Spring Creek Hatchery. The predominance of 3-year-old fish from Spring Creek Hatchery, nearly 80\% (Appendix Tables 5-8) recovered in the fisheries for the 1978 and 1979 broods greatly influenced the age distributions for these two broods. When the contribution from Spring Creek Hatchery dropped dramatically for the 1980-brood fish, the contribution proportion for WDF facilities increased (Figure 17). The WDF facilities appear to produce fish that contribute more heavily as 4-year-olds (Figure 9). The Spring Creek Hatchery and ODFU facilities contribution proportions increased slightly for the 1981 brood, and except for Priest Rapids Hatchery, contribution'proportions from WDF facilities decreased slightly (Figure 18). Like Spring Creek, fall chinook salmon from ODFW facilities appear to contribute to the fisheries more heavily as 3-yearold fish (Figure 9). 
It is not clear why fall chinook salmon from WDF facilities appear to contribute to the fisheries more heavily as 4-year-olds and those from USFWS and ODFW facilities contribute more heavily as 3-year-olds. It is possible that there is a genetic reason for the apparent later maturity of the fish from WDF facilities. However, with the lower Columbia River stock of fall chinook salmon (Tules) there has been considerable transfer of fish from ODFW facilities (Big Creek and Bonneville) and USPWS facilities (Spring Creek and Abernathy) to all WDF facilities except Priest Rapids Hatchery. This in combination with straying of fish among facilities would seem to eliminate any genetic differences among the fall chinook salmon reared at the facilities. Fish from Priest Rapids Hatchery are the upriver bright stock, which is genetically different than the Tule stock (Milner et al. 1985). Thus, there does appear to be a genetic basis for the different fishery and age distribution of fall chinook salmon from Priest Rapids Hatchery when compared to fall chinook salmon from other rearing facilities on the Columbia River.

A possible reason for the apparent later maturity for the Tule stock of fall chinook salmon from WDF facilities is that the fish from WDF facilities tend to be released later and at a smaller size than Tule stock from ODFW and USFWS facilities (see Tables 1 - 4). This smaller size and later release may result in less ocean residence time during the first year of ocean life and possibly lead to later maturity.

Comparison of the age distribution of the 1978 through 1981 broods of fall chinook salmon with those for the 1961 though 1964 broods May be confounded by the difference in marking methods and the size of the fish released. Fin and maxillary bone removal was used to mark fall chinook salmon during the 1961- through 1964-brood study. There was same indication that the marking may have delayed maturity causing the fish to stay in the ocean a year longer than the unmarked fish. The fish in the earlier study were also released at a smaller size, 220 to 661 fish/kg (100 to $300 \mathrm{fish} /$ pound) than those in the 1978 - 1981 study (Wahle and Vreeland 1978). Release of smaller fish may tend to \&lay maturity and cause the fish to stay in the ocean a year longer. Despite these potential problems, the trend for the two studies was similar with about $60 \%$ of the fall chinook salmon from the 1961- through 1964-brood study being recovered as 3 -year-olds and about $30 \%$ being recovered as 4- year-olds.

Comparisona among hatcheries between the 1961 - 1964 study and the 1978 - 1981 study are not possible because the marking limitation (number of distinct marks available which were thought to have limited effect on survival of the fish) prevented individually identifying the production from each hatchery in the earlier study. However, two hatcheries were special marked (Spring Creek and Kalama River hatcheries) during all four brood years of the 1961 - 1964 study. For the 1961 - 1964 study, Spring Creek Hatchery fish contributed most heavily as 3-year-olds (62 to $70 \%$ ). In the 1978 - 1981 study, 3-year-old recoveries of Spring Creek Hatchery fish ranged from 77 to $82 \%$ (Appendix Tables S-8). The lower proportion of 3-year-olds in the earlier study may have been due to the smaller release size and the delayed maturity impact of the mark; removal of the adipose and left ventral fins and a portion of the maxillary bone (Wahle and Vreeland 1978). 
For the Kalama River Hatcheries (Kalama Falls and Lower Kalama combined), in the earlier study, the 3-year-old recovery proportion ranged from 20 to $52 \%$ and the 4-year-old recovery proportion ranged from 34 to 63\%. Fishery recoveries of 3-year-olds predominated for two broods (1962 and 1963) and recoveries of 4-year-olds predominated for the other two broods (1961 and 1964) (Wahle and Vreeland 1978). For the 1970 - 1981 study, the 4-year-old recovery proportion predominated for all four broods from Kalama Falls Hatchery and two out of three broods from Lower Kalama Hatchery (Appendix Tables S-8). If the marking method and smaller release size of the 1961- 1964 study delayed maturity by a year, one would expect the 4-year-olds to be predominant for all brood years in the earlier study. Thus the delayed maturity due to release. size and marking method in the earlier study is not well supported by the comparison of the two studies.

\section{Fishery Contribution Estimation}

The contribution estimates presented in Tables 13 though 16 are considered minimum values for several reasons. First, most, but not all releases from the facilities were represented by tagged fish. Second, . marking and tagging took place at some facilities for purposes other than the contribution study, and these other marked or tagged groups have not been included. Finally, fishery sampling took place in the major marine and freshwater fisheries, but some fisheries were not sampled (Alaska sport fishery) or observed recoveries could not be expanded (British Columbia sport fishery). There is a potential for recoveries of tagged fall chinook salmon from this study in these fisheries.

The reasons for the apparent differences in fishery contribution and survival among broods are not abundantly clear. The most likely reason would seem to be conditions in the ocean. The influence of ocean conditions on survival of salmonids has been postulated by many fishery scientists (Pearcy 1984). Factors such as ocean upwelling (Gunsolus 1978), predation (Varoujean and Matthews 1983). and sea surface temperature (Mathews 1984) May all play a part in survival of salmonids. The Conditions the four broods of fall chinook salmon faced after release were very different. The 1978 brood, released in 1979, entered what might be termed normal conditions. The 1979-brood fall chinook salmon migrated downstream in the Columbia River before, during, and after the eruption of Mt. St. Helens on May 18. 1980 . The 1980 and 1981 broods were in the ocean during the El Nino of 1982 and 1983 (Buyer and Smith 1985). The El Nino influenced marine conditions as far north as the Queen Charlotte Islands in British Columbia (Tabata 1985). If one were only looking at these factors, it would appear the the eruption of ht. St. Helens had a positive effect on survival and the El Nino had a negative effect. However, individual rearing facility survivals indicate this is a too simplistic view since fall chinook salmon from all facilities did not react to these events in the same manner.

Stayton Pond contributions and survivals remained stable for the first two brood years (6.7 fish caught per 1000 releases and 0.6 and $0.8 \%$ total survival for the 1978 and 1979 broods respectively) and then 
dropped for the last two broods $(2.8$ and 3.5 fish per 1000 releases and 0.3 and $0.5 \%$ survival for the 1980 and 1981 broods respectively) (Tables 13 - 21). Nothing in the rearing and release data provide a clue as to the reason for the differences in contribution and survival. Stayton Pond is located on the Willamette River system. Fish are not released directly from the pond. They are collected and transported by truck to various Willamatte River tributaries. The releases took from two to six weeks with avarage release sizes in the 70 to 90 fish per pound range for the four broods. From 4.7 to 6.7 million fish were released each year and no diseases were noted. Measurements of the status of smoltification were not mada at Stayton Pond. Recoveries of migrating tagged fish were nade at Jones Baach for all four broods. Numbers of recoveries at Jones Reach do not imply the differences in survival are due to freshwater conditions. The protracted release periods eliminate the possibility of examining migration rate to Jones Beach as an indication of smoltification status. It appears the El Nino event had a negative impact on the survival of fall chinook salmon from Stayton Pond.

Spring Creek Hatchery showed a trend similar to that for Stayton Pond. The one difference is that 1979-brood fish from Spring Creek Hatchery had the greatest survival of all broods and rearing facilities Releases from Spring Creek Hatchery were made at four different times and sizes for all four brood years: March, April, May, and August. Release times and sizes were about the same each year There is some indication that suggests development of - mollification may play a part in survival. The quantity of an enzyms (ATPase) in the gills of the fish increases as salmonids near the time of migration and entry into sea water (Zaugg and McClain 1970). Measuremants were mada of the quantity of ATPase in the gills of fall chinook salmon during the rearing period throughout this study. ATPase levels had begun to increase prior to any of the releases of 1978- and 1979-brood fish (Prentice et al. 1980 and 1981). The enzyme level did not incraase prior to release of any of the 1980-brood fish and did not increase in the 1981 brood until just before the May release (Zaugg [In press]). The 1981-brood fish from the May release had the greatest fishery contribution rate and survival of any fall chinook salmon released from rearing facilities -for the 1980 and 1981 broods.

Levels of ATPase were not collected at all facilities for each brood year. Thus it is impossible to know if development of ATPase played a part in Abernathy, Sea Resources, Priest Rapids, Cowlitz, Elokomin and Lower Kalama hatcheries having their greatest contributions for the 1980 and 1981 broods, when the average contributions for these broods were at the lowest values. There are no hatchery data that provide a clue as to why some facilities had their best contributions to the fisheries in years when other facilities had their lowest contributions. There are two potential reasons for the greater survival of 1980- and 1981-brood fall chinook salmon from Priest Rapids Hatchery. The first reason is that the fish from Priest Rapids Hatchery are a different stock of fall chinook salmon, upriver brights. The Priest Rapids stock tend to have a more northerly distribution than the lower river stock as shown by the greater proportional contribution of Priest Rapids fish to the Alaska fisheries. It is possible the more northerly distribution 
provided a survival advantage for fish from Priest Rapids Hatchery during the El Nino years.

Another potential reason for the greater survival of the later broods from Priest Rapids Hatchery is the transportation program at McNary Dam. McNary Dam is the first dam below Priest Rapids Hatchery. The experimental phase of transportation of salmonids from McNary Dam to below Bonneville Dam began in 1978. Downstream migrating salmonid smolts were diverted from the turbine intakes at McNary Dam by submerged traveling screena. These diverted fish were collected in raceways at the dam and then placed in tank trucks or barges for transport downstream and release below Bonneville Dam. In 1979 and 1980 when the 1978 and 1979 broods of fall chinook salmon from Priest Rapids Hatchery were migrating downstream, about 100,000 fall chinook each year were transported from McNary Dam (Park 1985). In 1981, transportation at McNary Dam became an operational program(Athearn 1985), when research results indicated a positive survival benefit for transported salmonids, particularly fall chinook (Park 1985). In 1981 and 1982 (during the migration of the 1980 and 1981 broods) 2.1 and 1.7 million fall chinook salmon smolts respectively were transported from McNary Dam (Athearn 1985). One could assume that this transportation had some positive benefit on the contribution and survival of the 1980 and 1981 broods $0 f$ fish from Priest Rapids Hatchery. The extent of the transportation benfit is unclear because the number of Priest Rapids Hatchery fish tranaported is unknown. The number is a factor of the fish guiding efficiency of the turbine screen at McNary Dam, the size of fish releaed and the time of release from Priest Rapids Hatchery, and spill at McNary Dam.

In general for the 1978 and 1979 broods, the hatcheries which released fish prior to June at a size larger than $220 \mathrm{fish} / \mathrm{kg}$ (100 fish/pound) had the greatest survivals. This trend is not clear for the 1980- or 1981-brood fish. For the 1980 brood there appeared to be a possible trend of the larger the fish at release the better the survival. The size/survival relationship was not clear for the 1981 brood.

The ability to determine the reasons for the apparent differences in fishery contributions and survivals among broods, rearing facilities within broods, or releases within rearing facilities is limited in this study The study was not designed to determine the reasons for differences, but to indicate if differences did in fact exist and suggest how great the differences might be. Survival influencing factors likely include rearing environment, stock of fish, time of release, size of release, health of fish, status of smoltification at release, release location, and ocean conditions. Analysis of the factors which may affect survival is complicated by the limited data available. Examination of some factors is limited to noting an occurrence. For example, there are no quantitative measures for disease history. It is not possible to make a comparison between groups of fish where group $A$ is known to be $X$ percent healthier than group $B$. The most that can be done is to note group $A$ had certain diseases and group $B$ had none. Thus the disease history differences would be a potential reason for survival differences. Because of the confounding nature of the factors which may influence survival, isolating one or more of them as 
key factors is impossible given the rearing, release and recovery data available.

It seems likely the 1982 - 1983 El Nino would have a profound influence on the survival of salmonids in the northeast Pacific. The apparent reductions in overall contributions and survivals of the 1980 and 1981 broods of fall chinook when compared to the 1978 and 1979 broods are likely an indication of the influence of the $\mathrm{El}$ Nino. However, rearing facilities and release within the 1980 and 1981 broods were not equally effected. This indicates factors affecting the fish prior to ocean entry may also play an important part in the subsequent contribution and total Survival.

The average fishery recovery to release ratio for the 1961 - 1964 broods of fall chinook salmon from Columbia River hatcheries was $6.7 \mathrm{fish}$ recovered per 1,000 releaes. The ranges by brood were 3.1 to 10.0 (Wahle and Vreeland 1978). The average for the 1978 - 1981 broods is over three times less than for the past study (1.9 fishery recoveries per 1,000$)$. The greatest fishery recovery ratio for a brood (4.7 fishery recoveries per 1,000 releases for the 1979 brood) is under half the greatest fishery recovery rate for the put study. Thus, it appears the 1978 - 1981 broods of fall chinook salmon from Columbia River hatcheries did not contribute as well to the fisheries a in the past Study The greatest fishery recovery ratio for the put study for any one hatchery was for the 1964-brood fish from Spring Creek Hatchery: 26.5 fishery recoveries per 1,000 releases (Wahle and Vreeland 1978). For this study, the greatest ratio also came from Spring Creek Hatchery, but was only 12.7 fishery recoveries per 1,000 releases.

A partial reason for the decline in fishery recoveries of fall chinook salmon is the changes in the fishery regulations designed to reduce catches of these fish in the marine fisheries. These changes were taking place in the ocean fisheries off the coasts of all the Pacific coast states and British Columbia in the early to mid 1980's (Anon. 1984, Davis and Seibel 1988). The other reason for the decline is a lower survival of fall chinook salmon from Columbia River hatcheries. This will be discussed in more \&tail later under 'Total Suntival".

Rearing Facility and Adjacent Stream Returns

Fishery contribution to escapement and survival estimates presented in Tables 17 through 21 should be used with caution. Escapement values are minimums for two reason Sow adult traps at return facilities are not effective at recovering returning 2-year-old fall chinook salmon (Kalama Falls and Lower Kalama hatcheries). At other hatcheries some fish may be passed upstream to spawn or be caught in special fisheries (Kalama Falls and Washougal hatcheries). Stream surveys could not observe or recover all fish in the stream. Expansion factors for unsampled fish were not applied to the stream survey data by all recovery agencies. To maintain consistency, the stream survey data included in the escapement in this report are for observed recoveries of tagged fish only.

The reasons for the apparent differences in age proportions in the 
returns and in the fisheries are not completely clear. There appear to be several compounding factors. One might expect to see a higher proportion of 2-year-old fall chinook salmon in the returns than in the fishery recoveries because of catch size limitations in the ocean fiaheries and mesh size limitationa in the Columbia River commercial fisheries. This is the case for some facilities but not for others. The inefficiency of some facilities in trapping 2-year-old fish at return confounds the expectation. The difference in sampling scheme in the fisheries and at return likely plays a part in the difference in proportiona. The fisheries were randomly sampled at a $15 \%$ to $20 \%$ rate. In moat cases all returns to rearing facility are sampled for tagged fish. With small numbers of 5- and 6-year-old fish, the sampling scheme at the rearing facilities would be more likely to detect the older returns. The ability of adult fish to reach the rearing facilities because of stream flow may be a confounding factor. Straying of adults to adjacent streams, where only observed data were used likely also contributes to the differences between the age proportions in the fisheries and at return.

There is also a possibility that fishery selectivity may play a part in the apparent differences in ages in the fishery contribution and at return. There are four likely ages for returning fall chinook salmon, . 2-, 3-, 4-, and 5-year-olda. It is not known how the fall chinook salmon are mixed in the fisheries. One might suspect that fish from a rearing facility remain a a loose group in the ocean until part of the group reaches a certain stage of maturity. At that point, the more mature fish would leave the population and return to the natal stream. In this case unequal fishing pressure could occur on the separated groups during any one year. For example, 3-year-old fall chinook salmon from a Columbia River rearing facility could be recovered in september in the Columbia River fisheries, during the spawning migration, and in Washington troll fisheries during the same time period. The two groups of fish could be subject to different fishing pressures due to different locationa, types of gear, and fishing regulations. The same unequal fishing pressure may occur in the ocean depending on the amalgamation of fish, location of the groups, and when the maturing fish leave the rest of the population. Unequal fishing pressure on separated groups of fall chinook salmon from the same rearing facility might result in a different age structure in the fishery contribution than at return to the rearing facility.

The reason for the extent of straying of tagged fall chinook salmon from this study are not well understood. In some cases, homing to the parent stream has been found to be quite exact (Rounaefell end Kelez 1938; Taft and Shapovalov 1938; Donaldaon and Allen 1957; Ellis 1968; Jensen and Duncan 1971; Mahnken and Joyner 1973; Vreelend, Wehle, and Arp 1975, Scholz et al. 1976; Vreeland and Wahle 1983). Olfaction is reported to be important in the homing of adult salmon (Hasler and Wisby 1951; Wisby and Hasler 1954; Groves, Collins, and Trefethen 1968). It has been theorized that the homing imprint is acquired rapidly before and/or during downstream migration (Hasler 1966; Carlin 1968; Wagner 1969; Hasler, Scholz, and Horrall 1978).

There are reports in the literature of sizable numbers of straying salmon (Shapovalov and Taft 1954) and straying in non-natal river for a 
considerable distance (McLeod and O'Neil 1983). Quinn and Fresh (1984) reported a $1.4 \%$ straying rate for spring chinook salmon from the Cowlitz River Hatchery.

There are several factors which may play a part in the extent of straying in this study. Many of the past studies on homing have been conducted with coho salmon (Oncorhynchus kisutch) and steelhead trout (Oncorhyzxhus mykiss). These species are normally released as yearlings and at a larger size than the sub-yearling fall chinook salmon (Wahle and Smith 1979). The imprinting of fall chinook salmon may occur at a different time and/or over a longer period of time. Also, past work with salmon homing did not have the advantage of the coded wire tag, which allows exact identification of the returning fish. This marking method may reveal straying that past methods (primarily fin removal) may not have shown.

It is also possible that the release environment may play a part in the extent of straying. It appeared the greatest amount of straying occurred among facilities that released into tributaries emptying into the Columbia River estuary. The tidal influence in the estuary may move the fish around the estuary as the imprinting process is taking place. There is some evidence that some fall chinook salmon moved upstream upon entering the estuary. There were some recoveries at Jones Reach of fish. releued into tributaries entering the estuary below the JOMS Reach sampling site (Tables 5 and 6 ). A number of stray fall chinook salmon entered Bonneville Hatchery imediately below Bonneville Dam. The delay of adult salmon caused by Bonneville Dam may contribute to the number of strays at Bonneville Hatchery. Another factor which may contribute to straying is the low stream flows in the fall when fall chinook salmon return to spawn. Low-flows at certain times in the parent stream may cause the salmon to seek other spawning sites. Also, once a stray fish enters a hatchery or trap, it \&es not have the option to leave to seek the parent spawning site.

Olfactory nerve \&generation has been noted in chum (Oncorhpchus keta) and chinook salmon caused by misplacement of the coded wire tag (Morrison and Zajac 1987). It is possible this type of damage alters the homing behavior of salmon. Misplacement of coded wire tags would be more likely in fish tagged at a small size, as is the case with fall chinook salmon.

It is clear some fish turn off into tributaries long before reaching the parent stream (Washougal Hatchery $f i s h$ in the Kalema River), and other fish bypass the release facility (Priest Rapids Hatchery fish at Wells Dam). It is also clear that some fish make large homing errors, as evidenced by the two returns of Columbia River fall chinook salmon outside the Columbia River system. This number is extremely small considering the number of hatcheries involved in this study and the numbers of tagged fish released. In a study at Cowlitz Hatchery with spring chinook salmon, by comparison, 10 fish strayed to areas outside the Columbia River over four brood years (Quinn and Fresh 1984). It is interesting to note that the fall chinook salmon straying outside the Columbia River returned to hatcheries located on tributaries at the southern end of the Puget Sound and Willapa Bay. 
Total Survival

Total survival estimates in this report are minimums because the fishery contribution estimations are minimums for the reasons described under 'Fishery Contribution" and the escapement numbers include only observed recoveries from stream surveys.

It is not clear why the 1980 brood fish had lower fishery recovery ratio but higher survival when compared to the 1981 brood. The El Nino could have had a greater impact on the 1981-brood fish than the 1980 brood because it occurred during the first year of ocean life for the the 1981 brood. This accounts for the lower survival of the 1981 brood. But changes in the fishery regulations, beginning in 1984 to reduce the catches of fall chinook stocks, did not have an impact in the British Columbia fisheries until 1985. The regulations reduced the recoveries off the Washington coast in 1984, but the ocean recoveries in British Columbia were greater in 1984 than 1983. This was particularly true for the Vancouver Island fisheries where the the recovery in 1984 was over 120,000 fish greater than in 1983 (Pacific Salmon Comission 1986). The Vancouver Island fisheries recover the greatest proportion of the Columbia River chinook salmon in British Columbia. Regulation changes . did not begin to impact the British Columbia fisheries until 1985 (Pacific Salmon Comiaaion 1986). The British Columbia fisheries in 1983 and 1984 would have impacted the 3 and 4-year-old fish of the 1980 brood, which account for $90 \%$ of the fishery recoveries, and the 2- and 3-year-old fish of the 1981 brood. The fishery regulations and recoveries would seem to imply that the fishery contribution to release ratio would be higher for the 1980 brood than the 1981 brood rather than the opposite.

The survival estimates for fall chinook salmon from Columbia River rearing facilities are lower for this study than for the 1961- through 1964-brood study. The average survival for the 1961 - 1964 study was 0.007 , with a range among broods from 0.003 to 0.011 (Wahle and Vreeland 1978). Thus, there appears to have been a reduction in survival of fall chinook salmon from Columbia River rearing facilities between the 1961 1964 brood study and this one.

The reduction in survival from the 1960's study to the present study was not expected. Fall chinook salmon were released at a larger size during the 1978 - 1981 brood study. The rearing conditions at the facilities were believed to be improved from the earlier study. These factors have been shown to improve the survival of salmon (Fowler and Banks 1980; Fowler et al. 1980; Fowler et al 1980). However, the reduced survival of chinook salmon stocks since the late 1960's and early 1970's is not unique to Columbia River rearing facilities. Fresh. Schroder, and Shepard (1987) determined the fall chinook salmon survival in the Columbia River reached a peak in 1967 and began a steady decline in the late 1960's or early 1970's. Wild and hatchery fish were both subject to this decline in survival. Survival of chinook stocks in Oregon coastal rivers as well'as the Columbia River were reduced by the El Nino in 1983 (Johnson 1984). Sandercock (Pers. com.) ${ }^{1}$ indicated hatchery stocks in British Columbia had undergone a decline during the same period as the Columbia River fall chinook salmon. The El Nino also had 
a negativp impact on survival of hatchery stocks in southern British Columbia.

Benefit/Coat Estimation

Capital costs were not included in the benefit/coat analyaia because in moat cues they were expendtd over 20 years ago and have already been amortized. The second reason for not including capital costs is that capitol coats cannot be recovered as long as the facilities cannot be used for rearing some other species of higher benefit. The facilities were Constructed as mitigation for Specific Species of Salmonids. Elimination of one species would in most cases not be politically or socially acceptable, even if that species had a poor benefit to cost ratio in relation to another species.

The benefits are believed to be minimums because the true total catch of salmon cannot be assessed due to the difficulty and expense of sampling all fisheries and landing sites for salmon on the Pacific coast of North America.

Benefit to coat ratios were not calculated for Lawis River and Speelyai. hatcheries because the fall chinook salmon rearing program at these facilities were experimental. Wild chinook salmon fry and fingerlings were captured in the Lewis River with a seine and reared at the hatcheries (McIaaac 1980). Thus costs are not equivalent to operations at other rearing facilities. Coats of rearing fall chinook salmon at Ringold Pond were combined with those for Priest Rapids Hatchery since the same tag code was used for both facilities, preventing allocation of the fishery recoveries by facility.

Comparisons of benefits among rearing facilities within and between brood years may be somewhat misleading. In some cases, a facility may have a greater contribution to the fisheries than another facility, but a smaller benefit (e.g. Washougal Hatchery vs. Big White Pond, 1978 brood). In other cases, the contribution may increase from one brood to the following brood from a single facility, but the benefit \&creases (e.g. Cowlitz Hatchery, 1979 to 1980 broods). These anomalies are a result of the diatribution of the catch for the facilities. With the contribution valuation method used in this report, the value of a sport caught fish is four to five times greater than the value of a conaercially caught fish. In the case of the Washougal vs. Big White Pond comparison, the sport fisheries accounted for $21 \%$ of the Big White contribution and $15 \%$ of the Washougal contribution. Thus the weighted average value per fish was $\$ 38.92$ for Big White and $\$ 32.44$ for Washougal. This resulted in a $\$ 8,100$ greater benefit for Big white than Washougal with 929 less fish in the estimated contribution to the fisheries. Therefore, the reader must be wary of making a judgment about facilities based solely on the estimated benefits for those facilities.

i Sandercock, K. A. Department of Fisheries and Oceans, Vancouver, B. C. Personal communication, Sept. 28, 1989. 
Comparison of benefit/coat ratios in this study with those in the 1960's study is made difficult by the inclusion of capital costs in the previous study, a differences in fish values, and methods of determining these values. The average benefit/cost ratio for the 1960's study was 4.2 to 1 . The ratios ranged between 2.0 to 1 to 7.2 to 1 among the four broods. By hatchery, the ratio ranged from 0.3 to 1 for Elokomin Hatchery (1961 brood) to 17.1 to 1 for Spring Creek Hatchery (1963 brood) (Wahle and Vreeland 1978). Thus, despite the lower catches and survivals for the fall chinook salmon from this study, compared to the previous study the benefit/coat ratios were similar.

\section{SUMMARY AND CONCLUSIONS}

In 1979 a coded wire tagging study was initiated at fall chinook salmon rearing facilities on the Columbia River system in Oregon and Washington. The purpose of the study was to examine the diatribution, contribution to the fisheries. survival, and value of fall chinook salmon from these facilities. Tagging of fingerling fall chinook salmon took place at 18 to 20 facilities each year from 1979 to 1982. Nearly 14 million tagged fish were released for the four brood years.

Approximately $4 \%$ of the production releases of fall chinook salmon were tagged. Tagged fish were recovered in Columbia River estuary sampling from 1979 through 1982. Fishery recoveries and spawning returns occurred from 1980 through 1986.

Tagged fish from moat groups of fall chinook salmon released at locations above the estuary sampling sites were recovered in the estuary. Recoveries ranged from none for some groups to over 2200 for one tagged group. Estuarine recoveries did not directly represent numbers of fish in the river due to factors affecting catch efficiency. These factors include size of fish at release, release location and time, migration behavior differences among different release groups, river flow, and survival of marked groups. Because of these factors, estuarine recoveries were generally not an indicator of adult survival of subyearling fall chinook salmon tagged for this study.

The fishery diatribution followed the same pattern as in past studies of fall chinook salmon from Columbia River rearing facilities in that the fish contributed primarily to fisheries north of the Columbia River mouth and to commrcial gillnet fisheries in-the Columbia River. The average fishery contribution over the four brood years was $3.6 \%$ to Alaska. 42.4\% to British Columbia, $27.0 \%$ to Washington, $4.7 \%$ to Oregon, $0.3 \%$ to California, 21.9\% to Columbia River, and $0.1 \%$ incidental recovery in foreign fisheries in the Pacific $\mathbf{O}$ cean off the $\mathbf{N}$ orth American coast. The contribution appeared to be more northerly for the 1980 and 1981 broods. This was apparently caused by institution of quotas and reductions in fishing time in the Washington ocean fisheries and warmer ocean water (El Nino) moving the fish farther to the north in 1982 and 1983.

The fishery contribution by age of fish is similar to that in the 1961through 1964-brood study. The contribution of 3-year-old fish predominated $(64 \%)$, followed by 4 -year-olds $(24 \%), 2$-year-olds $(8 \%)$. and 
5-year-olds (3\%) for the 1978 through 1981 broods combined. When rearing facilities are grouped by release agency, the contribution by age appears to be different for USFWS and ODFW facilities and WDF facilities. For USFWS and ODFW facilities, the 3-year-old contribution predominates, 78 and $77 \%$ respectively. For WDF facilities, the 4-yearold contribution predominates, 52\%. The later release and smaller size of fish released from WDFacilities, compared to those from USFWS and ODFW facilities, appears to reduce the length of ocean reaidence during the first year. This shorter growth time during the first year in the ocean may result in a smaller average size for the fish from WDF facilities at the beginning of the second year in the ocean. The smaller size may influence the fishery contribution by age, the ocean distribution, and age of maturity of fish from WDF facilities.

There also appears to be a difference in contribution by age among brood years. The contribution of 3-year-old fish predominates in all four broods, but the predominance declines from nearly 70\% for the 1978 and 1979 broods to 55 and $61 \%$ for the 1980 and 1981 broods The contribution of 4-year-old fish increases from about $20 \%$ for the 1978 and 1979 broods to about 30\% for the 1980 and 1981 broods. The Apparent reason for the change in contribution by age across broods is the reduced contribution of Spring Creek Hatchery for the 1980 and 1981 broods. Spring Creek Hatchery accounted for over $50 \%$ of the total contribution from all Columbia River facilities for the 1978 and 1979 broods. This contribution dropped to about 20\% for the 1980 and 1981 broods. Nearly $80 \%$ of the fish from Spring Creek Hatchery contribute to the fisheries as 3-year-olds.

The total contribution to the fisheries from facilities involved in this study is estimated to be $1,020,800 \mathrm{fish}$. This is an average of $2.9 \mathrm{fish}$ contributed to the fisheries for every $1000 \mathrm{fish}$ released. The contributions per 1000 releases by brood are 3.3, 4.7, 1.9, and 2.0 for the 1978 through 1981 broods respectively. These fishery contribution rates are lower than those reported in a study of the 1961 through 1964 broods of fall chinook salmon from Columbia River rearing facilities. The reasons for the lower contribution for fish from this study are not well understood. Changes in the fishery regulations reducing the recovery of chinook salmon in some marine fisheries and a general coastwide trend of declining survival of chinook salmon since the early

1970 's both may play a part. The El Nino of 1982 and 1983 appears to have created ocean conditions which limited the $\bullet$ urvival of the 1980 and 1981 broods of fall chinook salmon from Columbia River facilities.

Fishery contributions per 1000 fish released varied widely among facilities. The greatest contribution for any facility was 12.7 fish per 1000 release, 1979 brood from Spring Creek Hatchery. The lowest contribution was 0.1 fish per 1000 releases, 1978 brood from Elokomin Hatchery. The range of contribution rates by brood are: 1978 brood 0.1. Elokomin Hatchery to 8.6, Spring Creek Hatchery: 1979 brood - 0.3, Little White Hatchery to 12.7, Spring Creek Hatchery: 1980 brood - 0.2, Little White Hatchery to 7.8, Abernathy Hatchery; and 1981 brood - 0.2, Little White Hatchery to 6.0, Sea Resources Hatchery.

For the 1978 and 1979 broods, the fishery contribution from Spring Creek Hatchery was 55 and $52 \%$ respectively of the total contribution of fall 
chinook salmon from Columbia River rearing facilities. Releases of fall chinook salmon from Spring Creek Hatchery represented 21 and $19 \%$ of the total releases for the 1978 and 1979 broods respectively. For the 1980 and 1981 broods, the contribution proportion from Spring Creek Hatchery dropped to 19 and 25\% respectively of the total recovery. The release proportiona were 18.5 and $16 \%$ of the total releases for the 1978 and 1979 broods respectively.

Individual rearing facilities did not neceaarily have contribution patterns similar to the brood year averages. Six facilities (Abernathy, Sea Resources, Priest Rapids, Cowlitz, Elokomin, and Lower Kalama) had their greatest contribution to the fisheries and survivals for the 1980 or 1981 broods. It is not clear why this occurred. Apparently fish seemingly subject to the same marine conditions can have different survivals. It is not known how factors such as size of fish at the time of entering the ocean, conditions in the ocean upon entry, smolt status, fish health, or differences in diatribution after the fish enter the ocean may influence the ultimate survival of fish.

The average age at return to the rearing facilities followed a pattern that appeared to be slightly different than the fishery contribution. The 3-year-old returns predominated $(46 \%)$, but not by as great a margin; The proportion of 2-year-old returns is $41 \%$ followed by 2-year-olds at . 7\%, and 5-year-olds at 6\%. By brood year, the 3-year-old returns predominate for the 1978 and 1979 broods, the 3- and 4-year-old return proportiona are about equal for the 1980 brood, and the 4-year-old returns predominate for the 1981 brood. A factor in the difference between age proportions in the fishery contributions and at return is likely a difference in sampling schemes. The fisheries were sampled randomly at a $15 \%$ to $20 \%$ rate. In moat cues, all the returns to a rearing facility were sampled for tagged fish. Sampling of returns to adjacent streams consisted of examining all fish that could be found. Other factors which may have contributed to the apparent differences in ages of contribution and return are: 1) inability of fish to return to some facilities at times of low stream flow, 2) inefficiency of some adult trapping facilities in capturing the smaller returning 2- and 3year-old fish, 3) straying of returns to adjacent streams where only observed recoveries were used as return numbers, and 4) the potential for fishery selectivity.

There is in some cuessubstantial straying of fall chinook salmon among the facilities involved in this study and to adjacent streams. In several cues the rate of straying was greater than $208 \%$ There are a number of potential reasons for the apparent high rates of straying. Fall chinook salmon in the Columbia River return to spawn in August and September when tributary streams are at their lowest flows. Low flows in streams where rearing facilities are located may cause returning fish to seek other spawning sites. The release environment may influence the homing imprint process. The greatest amount of straying appeared to occur among rearing facilities releasing into or just above the Columbia River estuary. The influence of the tidal fluctuations in the estuary may muddle the homing imprint process. Delay caused by Bonneville Dam is a probable reason for some straying. The ability to specifically identify fall chinook salmon for individual rearing facilities with a coded wire tag may have revealed straying rates which could not be 
identified in put studies using fin marks Poor placement of taga in the smallest fish might have caused olfactory nerve damage in some fish. This could have altered the homing behavior of these fish.

Total survival (fishery contributions and escapement to rearing facilities and adjacent streams) for all four broods combined is $0.33 \%$. By brood the survivals are 0.33\%, 0.46\%. 0.28\% and 0.25\% for the 1978 through 1981 broods respectively. Survivals among facilities across all broods ranged from a low of $0.01 \%$ for Elokomin Hatchery, 1978 brood, to a high of $1.47 \%$ for Spring Creek Hatchery, 1979 brood. It is not clear why the fishery contribution is higher for the 1981 brood than for the

1980 brood considering the survival for the 1981 brood is lower than that for the 1980 brood.

As with the fishery contributiona, the survivals of fall chinook salmon from this study were lower than those estimated for the 1961- through 1964-brood study. This was unexpected because the fish from this study were released at a larger size and thought to have been reared under better rearing conditiona than those from the earlier study. However, survival reductiona of fall chinook salmon have been noted by others on the Pacific coast over the past 15 to 20 years.

The total coat of rearing the 1978 through 1981 broods of fall chinook salmon for Columbia River rearing facilities is $\$ 6,334000$. The total benefit of the fishery recoveries of these fish is estimated at $\$ 36,242,200$. Thus the average benefit to coat ratio for all rearing facilities and all brood years combined is 5.7 to 1 . The range among broods is $2.5 / 1$ to $9.8 / 1$. The range among rearing facilities across all broods is $0.1 / 1$ to $26.6 / 1$. The benefits for the rearing facilities involved in this study are believed to be minimums for three reason Not every release group from every facility was repreaented by tagged fish. Some tagged groups were released from some facilities for purposes other than the contribution study. These groups were not included in the benefit analysis. The fishery contribution estimates are believed to be minimums because the Alaska marine sport fishery was not sampled for tagged fish and the tag recoveries in the British Columbia sport fishery could not be expanded to the total catch. There is a potential for recoveries of fall chinook salmon from Columbia River rearing facilities in both these fisheries.

This study was not designed to identify the factors which may lead to various rates of fishery contribution and survival. The purpose of the study was to determine what the fishery contribution rates and survival were for various brood years and rearing facilities and indicate if these rates differ among brood years and facilities. The data from this study indicate that fishery contribution and survival differ among brood years. Conditions in the ocean environment may be a major reason for these differences. The data also indicate that the conditions affecting fishery contribution and survival in a release year do not affect fall chinook salmon from all rearing facilities on the Columbia River in the same manner. For example, groups of fish with the same health, physical, and physiological status reaching the estuary, Columbia River Plume and near shore ocean at different times, might be subject to different environmental conditions. These different conditions may 
cause differences in survival. Groups of fish with different health, physical, and physiological - tatus may survive at different rates even though the environmental conditions are the same in the estuary, plume, and near shore ocean.

There is much analysis that could yet be done with the data collected for this study. Fishery contributions and survivals of individual release groups within a rearing facility are not analyzed in this report. Van Hyning (1973) and Fresh, Schroder. and Shepard (1987) ran correlations of factors which may affect survival of fall chinook salmon from Columbia River rearing facilities. This type of analysis could also be attempted with the data from this study. All of the rearing, release, and recovery data are available on computer to those who may have the inclination, time, and resources to undertake further analysis.

It is the authors opinion that a correlation of survivals with.various available rearing, release, and river, estuary, and wean conditions would be an interesting pursuit but this analysis would not yield definitive answers to the reasons for the aurvivala. There are certain pieces of data which are limited or not available. These data may play a critical roll in the survival of fall chinook salmon. For example, limited disease data are available in Appendix A. However, the data only tell when a disease was detected and what treatment was used. They do not necessarily indicate the health of the fish at release or the health in comparison to fish in which no or less diaease was detected. Despite years of research there is yet no way to quantify the health of fish released from rearing facilities. Other pieces of limited or missing data are the smolt status at release; time of Columbia River plume and/or ocean entry; food availability in the estuary, Columbia River plume, and ocean at and after the time of entry into these environments; the types and numbers of competitors and predators in the estuary, Columbia River plume, and near shore ocean; and survival rates of juvenile fish in the estuary, Columbia River plume, and during early ocean life. Without complete data for these factors, the value of a survival correlation may be limited. 


\section{ACKNOWLEDGLENTS}

Reglonal electrical rate payers, through Bonneville Power Adminiatration, provided the funds necessary to conduct this study. Many individuala are required to conduct a study of this magnitude. It is difficult to express my thanks to each individual having a part in the development, conduct, and reporting phases of this study. My apologies to anyone I may have inadvertently overlooked. My sincerest appreciation is expressed to hatchery personnel at UDF, ODFW, and USFWS facilities participating in this study. Roy Wahle and Don Worlund, formerly with NRFS, deserve credit for assistance with study design. Bill Hopley, Robert Foster, Paul Seidel, Andy Appleby, Howard Fuss, Gary Sherman, Wolf Daamers.with WDF; Harold Hansen, Dennis Isaac, Ron Willams, with ODFW; Steve Olhauaen, Craig Tuaa, with USFWS; and Jim Ceballoa, with RHFS all deserve special recognition for their parts in conducting this study. I am especially grateful to Robert 2. Smith, IWFS who constructed the tagging trailer used for much of the fish tagging in this study, designed the data base containing all the rearing, release, recovery, and return data, and provided constant aaaiatance with computer operation. The assistance of Martha Maten in data compilation and Jane Rucka, NMFS. in preparation of annual reports and draft text is also greatly appreciated. Earl Dawley, Richard Ladgerwood, and Wally Zaugg, NOM Northwest Fisheries Center; and Bill Hopley, WDF, provided helpful editorial comment. 
ALLEN R. L.

1970. Wells sumer chinook salmon spawning channel, annual report, 1969 - 1970 season. Unpubl. manuscr.., 23 p., Wash. Dept. Fish., Room 115, Gen. Admin. Bldg., Olympia, WA 98504.

\section{ANONYMOUS.}

1984. Report of the U.S./Canada technical comittee on chinook salmon. Unpubl. manuscr., 46 p., Pacific Salmon Comm., 600 1155 Robaon St, Vancouver, B.C. V6E 1 B9.

ATHEARN, J. B.

1985. A review of juvenile salmonid transportation operations from 1981 to 1984, p. 3-1 - 3-108. In U.S. Army Corps of Engineers [ed.] Comprehensive report of juvenile salmonid transportation. Unpubl. manuscr., 240 p., North Pac. Div., P.O. Box 2870, Portland, OR 97208

BRAN, 0. R., L. CARPENTER, J. PARKER, J. W. BIGGS, G. BOYINGTON, C. J. BUCK, E. W. KILLER, P. A. PARSONS, W. A. SCHOENFELD, AND V. B. STANBRRY.

1938. A study of comercial fishing operations on the Columbia River. Oreg. St. Plan. Bd., 73 p.

BERGMAN, P. K.

1968. The effects of implanted wire tags and fin excision on the growth and survival of coho salmon, Oncorhpchus kisutch (Walbaum). Ph. D. dissertation, Univ. Washington Seattle, 119 p.

BERGMAN, P. K., K. B. JEFFERTS, H. F. FISCUS. AND R. C. WAGER.

1968. A preliminary evaluation of an implanted coded wire fish tag. Wash. Dept. Fish., Fish. Res. Pap., 3(1);63-84.

CARLIN, B.

1968. The migration of salmon. p. 14-22. In Atlantic Salmon Association Centennial Award Fund, series of lectures. Salmon Assoc., Montreal, Que.

CRAIG, J. A. AND R. HACKER.

1950. The history and development of the fisheries of the Columbia River. Bull. U.S. Bur. Fish. $49(32): 133-216$.

CRATRAU, E. J.

1989. Lower Snake River compensation plan program annual report fiscal year 1988. Unpubl. manuscr., 38 p., U.S. Fish Wildl. Serv., lower Snake River Compen. Plan Office, 4696 Overland Road, Room 560, Boise. ID 83705.

COBB, J. N.

1930. Pacific salmon fisheries, p. 409-704. In C. F. Mayne [ed.] Report of the U.S. Commissioner of Fisheries for 1930. Bur. Fish. Doc. 1092, U.S. Dept. Commer. 
DAVIS, A. R. AND K. C. SEIBEL

1989. Report to the board of fisheries 1988 Southeast Alaska salmon troll fishery. In P. R. Larson [ea.] Report to the board of fisheries, Southeast Alaska and Yakutat (Region 1) 1988

finfish fisheries. Reg. Infor. Rep. 1589-02. Alaska Dept. Fish Game, Div. Comer. Fish., Juneau, AK, Jan. 1989.

DAWLEY, E. R., 8. D. LEDGERWGOD. T. H. BIAHK, C. W. SIMS, J. T. DURKIN, R. A. KIRN, A. E. RANKIS, G. E. KONAN, AND F. J. OSSIANDER.

1986. Migrational characteristics, biological observations, and relative survival of juvenile salmonids entering the Columbia River estuary, 1966-1983. Unpubl. manuscr., 256 p., Bonneville Power Admin., P.O. Box 3621. Portland, OR 97208.

DEIAM, K. R. AND R. Z. SMITH

1988. Columbia River Fisheries Development Program annual report for F.Y. 1987. U.S. Dept. Comer., Nat. Ocean. Atmos. Admin., Nat. Mar. Fish. Serv., NOM Technical Memorandum RKFS F/NWR-24, $78 \mathrm{p}$.

DONALDSON, L. R. AND G. H. ALLEN.

1957. Return of silver salmon, Oncorhynchus kfsutch (Walbaum) to point of release. Trans. Am. Fish. Soc. 87:13-22.

EAMES, M M AND M K. HINO.

1983. aN evaluation of four tags suitable for marking juvenile chinook salmon. Trans. Am. Fish. Soc. 112:464-468.

ELLIS, C. H.

1968. A return of adult coho salmon demonstrating a high degree of selectivity in homing. In Proceeding of the Northwest Fish Culture Conference, December 4-6, 1968, Boise, Idaho, P. 40-42. Unpubl. manuscr. Wash. Dept. Fish., 115 Gen. Admin. Bldg., Olympia, WA 98504.

FISH, F. F. AND M G. HANAVAN

1948. A report upon the Grand Coulee fish-maintenance project 1939-1947. U.S. Fish Wildl. Serv., Spec. Sci. Rep. 55, 63 p.

FRESH, K. L., S. L. SCHRODER, AND S. SHEPARD.

1982. Evaluating the production of fall chinook originating from the Columbia River. Unpubl. manuscr., 37 p., Wash. Dept. Fish., Room 115, Gen. Admin. Bldg., Olympia, WA 98504.

FOSTER, R. W.

1981. Incremental fish sampler. Prog. Fish Cult. 43:99-101.

POWLER. I. G. AND J. L. BANKS .

1980. Survival rates of three sizes of hatchery reared fall chinook salmon. U.S. Fish. Wild. Serv., Technology Transfer Series $80-1,12 \sim$. 
FOWLER, L. G., R. E. BURROWS, B. D. COMBS, AND A. E. THOMAS.

1980. The effect of improved rearing conditions on the adult survival of fall chinook salmon. U.S. Fish. Wild. Serv., Technology Transfer Series 80-2, 12p.

FOULER, L. G., R. E. BURROWS, B. D. COMBS, AND J. L. BANES.

1980. The effect of size and time of release of fall chinook salmon fingerlings on adult survival. U.S. Fish. Wild. Serv., Technology Transfer Series 80-3, 13 .

GROVES, A. B., G. B. COLLINS, AND P. S. TREFETHEN.

1968. Roles of olfaction and vision in choice of spawning site by homing adult chinook salmon (Oncorhynchus tshawytscha). J. Fish. Res. Board Can. 25:867-876.

GUNSOU1S, 8. T.

1978. The status of Oregon coho and recommendations for managing the production, harvest and escapement of wild and hatcheryreared stockas Unpubl. manuscr., 59 p., Oreg. Dept. Fish Wildl., 506 S.W. RI11 Street, P. 0. Box 3503, Portland, OR 97208.

HALLOCK R. J., G. H. WARNER, AND D. H. FRY, JR.

1952. California'a part in a three-state salmon fingerling marking. program. Calif. Fish Game $38(3): 301-332$.

HANSEN, H. L., R. D. EWING, AND J. T. MARTIN

1980. Federal aid progress reports fiaheriea 1979 Bonneville hatchery evaluation. Unpubl. manuacr., 22 p., Oreg. Dept. Fish Wildl., 506 S. W. Hill Street, P. 0. Box 3503, Portland, OR 97208.

HASIER A. D.

1966. Underwater guideposts:homing of salmon. Univ. Wis. Press, Madison, $155 \mathrm{p}$.

HASLER, A. D. AND W. J. WISBY.

1951. Discrimination of stream odors by fishes and its relation to parent stream behavior. Am. Nat. 85:223-238.

HASLER, A. D. A. T. SCHOLZ, AND R. h. HORRALL.

1978. Olfactory imprinting and homing in salmon. Am. Sci. 66:374355.

HAYES, H. L. AND K. A. HENRY.

1985. Salmon management in response to the 1982-83 El Nino event,

p. 226-236. In Wooster, W. S. and D. L. Fluharty [ea.] El

Nino north Nino effects in the eastern subarctic Pacific Ocean.

Univ. Wash., Sea Grant, USC-W0 85-3, 312 .

HEISING, P. L.

1972 Fish vs. dams: the economics of maintaining the Columbia

River basin fishery. Ph. D. dissertation, Wash. St. Univ., 251 P- 
HUME , R. D.

1893. Salmon of the Pacific coast. Univ. Microfilms. Inter., Ann

Arbor, Michigan, 52 p.

HUYER, A. AND a. L. SXITH.

1985. The apparition of El Nino event Oregon in 1982-83. p. 73-84.

In W. S. Wooster and D. L. Fluharty (ed.] El Nino north Nino

effects in the eastern subarctic Pacific Ocean. Univ. Wash., Sea Grant, USC-U0 85-3, 312 p.

JENSEN, A. L. and R. N. DUNCAN.

1971. Homing of transplanted coho salmon. Prog. Fish-Cult. $33: 216-218$.

JOHNSON S. L.

1984. The effects of the $1983 \mathrm{El}$ Nino on Oregon's coho and chinook salmon. Oreg. Dept. Fish Wlldl., Information Rep. 84-8, 40 p.

JUNGE, C. O. AND W. H. BAYLIFF.

1955. Bstlnating the contribution of a salmon production area by marked fish experiments. Wash. Dept. Fish., Fish. Res. Pap., $1: 51-58$.

LAIIIZ, I. I.

1948 The fishery development program in the lower Columbia River. Trans. Am. Fish. Soc. 78:42-55.

MAHNKEN, C. AND T. JOYNER.

1973. Salmon for New England fisheries, Part III: Developing a costal fishery for Pacific salmon. Mar. Fish. Rev. 35(10):9-13.

MATHEWS, S. B.

1984. Varlablllty of marine survival of Pacific salmonids: a review. p. 161-186. In W. G. Pearcy (ed.] The influence of ocean conditions on the production of salmonids in the North Pacific a workshop. Oreg. State Univ., Sea Grant, ORRSU-W83-001, 327 p.

MCISAAC, D.

1980. North Levis River wild stock fall chinook tagging project 1979. Unpubl. manuscr., Wash. Dept. Fish. Memorandum, 34 p., Wash. Dept. Fish., P.0. Box 999 MS S-13, Battleground, WA 986D4.

McLeoD, C. I. AND J. P. O'NeIL.

1983. Major range extensiona of anadromous salmonids and first record of chinook salmon in the Mackenzie River drainage. Can. J. Zo०1 61:2183-2184.

MEEKIN, T. K. AND A. C. MOSER.

1966. Rocky Reach fall-chinook salmon spawning channel 1964 brood annual report. Unpubl. manuscr., 27 p., Wash. Dept. Fish., Room 115, Gen. Admin. Bldg., Olympia, WA 98504. 
MILNER, G. B., D. J. TEEL, F. M. UTTER, AND G. A. WIMNS.

1985. A genetic method of stock Identification in nixed populations of Pacific salmon, Oncorhynchus spp. Mar. Fish. Rev. $47(1): 1-8$.

MORRISON, J. AND D. ZNAC.

1987. Histologic effect of co\&d wire tagging in chum salmon.

N. An. J. Fish. Manag. 7:439-441.

PACIFIC MARINE FISHERIES COMAISSION.

1988. Paciflc salmonid co\&d wire tag releases through 1987. Unpubl. namuscr., 228 p. Reglonal Hark Processing Center, Metro Center - Sulte 170, 2000 S.W. First Ave., Portland, OR 97201

\section{PACIFIC SAUYON COMAISSION.}

1986. Final 1985 report of the chinook technical comittee. Unpubl. - cr., 279 p. Pacific Salmon Com., 600-1155 Robson St., Vancouver, B.C. V6E $1 B 9$.

PARK, D. L.

1985. A review of smolt transportation to bypass dams on the Snake and Columbia rivers, P. 2-1 - 2-65. In U.S. Army Corps of Englneers [ed.] Comprehensive report of juvenile salmonid transportation. Unpubl. manuscr., 240 p., North Pac. Div., P.o. Box 287, Portland, OR 97208

PEARCY, W. G.

1984. The Influence of ocean conditions on the production of.' salmonids in the North Pacific a workshop. Oreg. State Univ., Sea Grant, ORESU-W-83-001, 327 p.

PRENTICE, E. F., C. MAHNKEN, K. GORES, W. WAKNITZ, W. ZAUGG, L. FOLMAR,

R. KING, J. MIGHELL, W. DICKHOFF, T. FLAGG, L. HARRELL, A. NOVOTNY,

D. DAMRAER, E. WOLD, AND R. VREELAND.

1980. A study to assess status of snoltification and fitness for ocean survival of chinook and coho salmon and steelhead.

Unpubl. manuscr., 393 p., Nat. Mar. Fish. Serv., NN Alaska Fish. Cent., 2725 Montlake Blvd. E..Seattle, WA 98112.

PRE NTICE, E. F. , C. V. W. MAHNREN, W. S. ZAUGG, L. C. FOLMAR, $W . W$.

DICXHOFF, F. H. HANITZ, K. X. GORES, L. H. HARRELL, A. L. NOVOTNY, T. A. Flagg, C. G. SAFsten, AND J. L. MIGHeLL.

1981. Assessment of smoltification and fitness for ocean survival (quality) of chinook and coho salmon and steelhead in the Columbia River and Puget Sound hatcheries. Unpubl. manuscr., 150 P., U.S. Nat. Mar. Fish. Serv., NW Alaska Fish. Cent., 2725 Montlake Blvd. E., Seattle, WA 98112.

QUINN, T. P. AND K. FRESH.

1984. Homing and straying in chinook salmon (Oncorhynchus tshawytscha) from Cowlitz River hatchery, Washington. Can. J. Fish. Aquat. Sc1. 41:1078-1082. 
RAYMOND, H. L.

1979. Effects of dams and impoundments on migrations of juvenile chinook salmon and steelhead from the Snake River, 1966 to 1978. Trans. Am. Fish. Soc. 108:505-529.

RAYYOND, H. L. AND C. H. SIRS.

1980. Assessment of smolt migration and passage enhancement studies for 1979. Unpubl. nanuscr., 143 p., U.S. Nat. Mar. Fish. Serv., NV Alaska Fish. Cent., 2725 Montlake Blvd. E. Seattle, WA 98112.

REINGOLD, M.

1978. Evaluation of transplanting Snake River steelhead trout to the Pashimero1 River, 1977. Unpubl. Manuscr., 17 p., Idaho Dept. Fish Game, P.0. Box 25, Boise, ID 83707.

$\mathrm{RICH}$,

1928. Experiments in marking young chinook salmon on the Columbia River, 1916 to 1927. Bur. Fish. Bull. 44:215-264.

RICHARDS, J.A.

1968. An economic evaluation of Columbia River anadronou fish programs. Unpubl. manuscr. Ph. D. dissertation, Oreg. St. Univ... $274 \mathrm{p}$.

RICHARDS, J. A.

1978. Estimating economic values for habitat management dec1siom. Unpub1. - C r. 34. p. Nat. Mar. Fish. Serv., 1002 R.R. Holladay St., Room 620, Portland, OR 97232.

ROUNSEFELL, G. A. AND G. B. KELEZ.

1938. The salmon and salmon fisheries of Swiftsure Bank, Puget sound, and the Fraser River. Bull. U.S. Bur Fish. 48:693-823.

SCHOENEMAN, D. E., L. C. SIRS, R. C. JOHNSEN, AND W. W. BENTLEY.

1961. Mortalities of downstream migrant salmon at McNary Dam. Trans. Am. Fish. Soc. 90:58-72.

SCHOLZ, A. T., R. M. HORRALL, J. C. COOPER, AND A. D. HASLER.

1976. Imprinting to chenical cues: the basis for home stream selection in salmon. Science (Wash., D.C.): 192:1247-1249.

SEAN, H.

1970a. Evaluation of $\mathbf{1 9 6 4}$ brood coho released from ten Puget Sound and one coastal hatchery. Unpubl. mamuscr., 40 p. Wash. Dept. Fish., Final Progress Rep., Room 115, Gen. Admin. Bldg., Olympia, VA 98504.

1970b. Evaluation of 1965 brood coho released from ten Puget Sound and three coastal hatcheries. Unpubl. manuscr., 42 p. Wash. Dept. Fish., Final Progress Rep., Room 115, Gen. Admin. Bldg., Olympia, WA 98504. 
1971. Evaluation of $\mathbf{1 9 6 6}$ brood coho released fromeleven Puget Sound and two coastal hatcheries. Unpubl. nanuscr., 42 p. Wash. Dept. Fish., Final Progress Rep., Room 115, Gen. Admin. Bldg., Olympia WA $\mathbf{9 8 5 0 4 .}$

SHAPOVALOV, L. AND A.C. TAFT.

1954. The life histories of the the steelhead rainbow trout (Salmo gairdnerl galrdneri) and silver salmon (Oncorhynchus kisutch). Callf. Fish Game Fish Bull. 98:375 p.

SIMS, C. W., J. G. WILliaks, D. A. FAUROT, R. C, JOHNSON, AND D. A. BREGE.

1981. Migrational characteristics of juvenile salmon and steelhead in the Columbia River Basin and related passage research at John Day Dam. Unpubl. - $c r$. , 362 p. U.S. Nat. Mar. Fish. Serv., wi Alaska Fish. Cent., 2725 Montlake Blvd. E.,Seattle, VA 98112.

SNYDER, J. 0.

1923. A second report on the return of king salmon marked in 1919 In the Klanath River. Cal1f. Fish Game 9:1-9.

TABATA, S.

1985. El Mino effects along and off the Pacific coast of Canada during 1982-83, p. 85-96. In W.S. Hooster and D. L. Fluharty [od.] El Nino north nino effects in the eastern subarctic Pacific ocean. Univ. Wash., Sea Grant, WSG-w0 85-3,312 p.

TAFT, A. C. AND L. SHaPOVAIOV.

1938. Honing Instinct and straying among steelhead trout (Salmon gelrdneril) and silver salmon (Oncorhynchus kisutch). Callf. Fish Game 24:118-125.

TUSS, C. A .

1982. John Day fall chinook altigation evaluation Spring Creek National Fish Hatchery broods 1972-1975. Unpubl. manuscr., 28 p., U.S. Fish W1ldl. Serv., Fish. Ass1se. Offlce, 9317 Hry. 99, Sulte I, Vancouver, WA 98665.

VAN HYNING, J. M.

1973. Factors affecting the abundance of fall chinook salmon in the Colubla River. Fish. Com. Oreg., Rea. Reports, 4(1) 87 p.

varoujen, D. H. AND D. R. Matmets

1983. Distribution, abundance, and feeding habits of seabirds off the Columbia River, Ray-June, 1982. Univ. Oreg. Inst. Mar. Blol., Charleston. Oreg. Rapt. No. OImB 83-1. 25 p.

VREELAND, R. R., R. J. WAHLE, AND A. H. ARP.

1975. Homing behavior and contribution to Columbia River fisheries of marked coho salmon released at two locations. Fish. Bull., U.S. 73:717-725. 
VREELAND, R. R. AND R. J. VAHLE.

1983. Homing and fisheries contribution of marked coho salmon, Oncorhynchus kisutch, released at two Columbia River locations. Fish. Bull., U.S. 81:143-148.

WAGNER, H. H.

1969. Effects of stocking location of juvenile steelhead trout, Salmo gairdneril, on adult catch. Trans. Am. Fish. Soc. 98:2734 .

WAHLE, R. J. AND R. 2. SMITH.

1979. A historical and descripeive account of Pacific coast anadromous salmonid rearing facilities and a sumary of their releases by region, 1960-76. Nat. Mar. Fish. Serv., Spec. Sci. Rep. Fish. 736, 40 p.

WAHLE. R. J., R. R. VREEIAND, AND R. H. LANDER.

1974. Bioeconomic contribution of Columbia River hatchery coho salmon, 1965 and 1966 broods, to the Pacific salmon fisheries. Fish. Bull., U.S. 72:139-169.

WAHLE. R. J. AND R. R. VREELAND

1978. Bioecononic contribution of Columbia River hatchery fall chinook salmon, 1961 through 1964 broods, to the Pacific salmon fisheries. Fish. Bull., U.S. 76:179-208.

HAHLE, R. J., R. O. KOSKI, AND R. 2. SMITH.

1979. Contribution of $\mathbf{1 9 6 0 - 6 3}$ brood hatchery-reared sockeye salmon, Oncorhynchus nerka, to the Columbia River commercial fishery. Fish. Bull., U.S. 77:229-242.

WISBY, W. J. AND A. D. HASLER.

1954. Effect of olfactory occlusion on migrating silver salmon

(O. kisutch). J. Fish. Res. Board Can. 11:472-478.

ZAJAC, D. P.

1985. A cursory evaluation of the effects of co\&d wire tagging upon salmonids. Unpubl. manuscr., 33 p., U.S. Fish. Wildl. Serv., Fish. Assistance Off., 2625 Parkmont Lane, Bldg. A, Olymia, VA 98502.

ZAUGG, $\mathbf{s}$.

(In press). Migratory behavior of underyearling Oncorhynchus cshargesche and survival to adulthood as related to prerelease gill $\mathbf{~ A a}^{+}-\mathbf{K}^{+}$ATPasedevelopment. Aquicult.

ZAUGG, H. S. AND L. R. MCCLAIN.

1970. Adenosine triphosphatase activity in gills of salnonids: seasonal variations and saltwater influence in coho salmon.

oncorhynchus kisu tch. Comp. Biochen. Physiol. 35:587-596. 